Old Dominion University

ODU Digital Commons

Physics Theses \& Dissertations

Physics

Summer 2018

\title{
Ion Bunch Formation Strategies for the JLEIC Collider
}

Bamunuvita Randika Prasad Gamage

Old Dominion University

Follow this and additional works at: https://digitalcommons.odu.edu/physics_etds

Part of the Elementary Particles and Fields and String Theory Commons, and the Nuclear Commons

\section{Recommended Citation}

Gamage, Bamunuvita R.. "Ion Bunch Formation Strategies for the JLEIC Collider" (2018). Doctor of Philosophy (PhD), Dissertation, Physics, Old Dominion University, DOI: 10.25777/q34c-ya06 https://digitalcommons.odu.edu/physics_etds/17

This Dissertation is brought to you for free and open access by the Physics at ODU Digital Commons. It has been accepted for inclusion in Physics Theses \& Dissertations by an authorized administrator of ODU Digital Commons. For more information, please contact digitalcommons@odu.edu. 


\title{
ION BUNCH FORMATION STRATEGIES FOR THE JLEIC COLLIDER
}

\author{
by \\ Bamunuvita Randika Prasad Gamage \\ B.S. June 2010, University of Colombo, Sri Lanka \\ M.S. August 2014, Old Dominion University
}

A Dissertation Submitted to the Faculty of

Old Dominion University in Partial Fulfillment of the

Requirements for the Degree of

DOCTOR OF PHILOSOPHY

PHYSICS

OLD DOMINION UNIVERSITY

August 2018

Approved by:

Todd J. Satogata (Director)

Geoffrey A. Krafft (Member)

Alexander Gurevich (Member)

Stephen Bueltmann (Member)

Desh Ranjan (Member) 


\begin{abstract}
ION BUNCH FORMATION STRATEGIES FOR THE JLEIC COLLIDER
\end{abstract}

Bamunuvita Randika Prasad Gamage

Old Dominion University, 2018

Director: Dr. Todd J. Satogata

An Electron-Ion Collider (EIC) has been proposed and supported as the next major nuclear physics facility in the United States, with very high design luminosities of $10^{33}-10^{34} \mathrm{~cm}^{-2} \mathrm{~s}^{-1}$. The Jefferson Lab EIC concept, JLEIC, includes construction of a new ion beam accelerator complex. To achieve the required luminosity, the JLEIC ion collider requires unprecedentedly short ion bunches of $1 \mathrm{~cm}$ length at a high repetition frequency of $476 \mathrm{MHz}$. However, only much longer ion bunches are formed by the ion injector complex producing beams for the collider ring. Formation of short ion bunches from much longer ion bunches in this collider while limiting beam size growth is a fundamental challenge. This dissertation investigates several methods using longitudinal RF gymnastics to address this bunch formation challenge, including debunch/rebunch with barrier bucket manipulations and adiabatic bunch splitting. We discuss background of both methods, and show results of simulations that indicate that multiple adiabatic bunch splits are best suited to produce ion beam that satisfies the current JLEIC requirements. Adiabatic bunch splitting is also further optimized using genetic algorithms to find the optimum tradeoff between acceptable emittance growth and accelerator time necessary to perform the bunch formation process. 
Copyright, 2018, by Bamunuvita Randika Prasad Gamage, All Rights Reserved. 


\section{ACKNOWLEDGEMENTS}

I would first like to thank my research adviser Dr. Todd Satogata for providing me with this project and the guidance to complete it. I would also like to thank Drs Alexander Gurevich, Geoffrey Krafft, Stephen Bueltmann and Desh Ranjan for serving on my committee and providing valuable feedback during the thesis evaluation meetings.

I'd like to extend additional thanks to Dr. Balša Terzić for his help setting up the genetic algorithm used for the optimization and providing continuous support with the issues that occurred. Thanks also to Dr. Jiquan Guo for several discussions about the different methods used to achieve the required number of bunches. A special thank you goes to my fellow graduate students Subashini DeSilva, Kirsten Deitrick and Hashir Rashad for sharing their knowledge and support.

This project was funded by the Thomas Jefferson National Accelerator Facility. Many thanks to Drs. Hari Areti and Michael Spata for their efforts to acquire the funding for the project

Finally I would like to express my deepest gratitude my family and friends who have provided me with support and encouragement the entire time. 


\section{TABLE OF CONTENTS}

Page

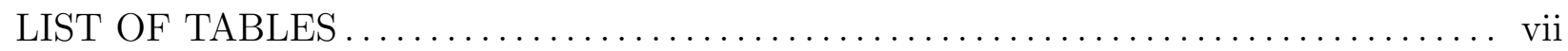

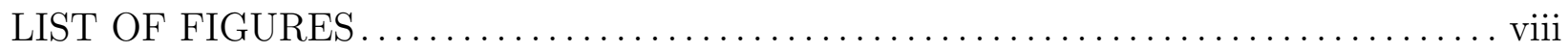

Chapter

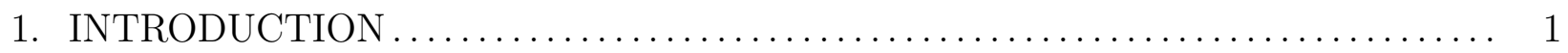

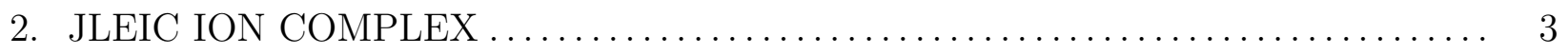

$2.1 \quad$ JLEIC ION INJECTOR COMPLEX $\ldots \ldots \ldots \ldots \ldots \ldots \ldots \ldots \ldots \ldots \ldots$

$2.2 \quad$ JLEIC ION COLLIDER RING $\ldots \ldots \ldots \ldots \ldots \ldots \ldots \ldots \ldots \ldots \ldots$

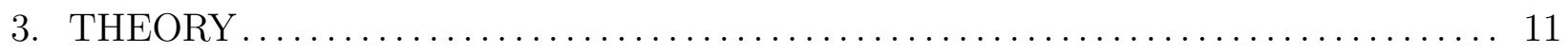

3.1 COORDINATE SYSTEMS AND PHASE SPACE COORDINATES $\ldots . \ldots \ldots .11$

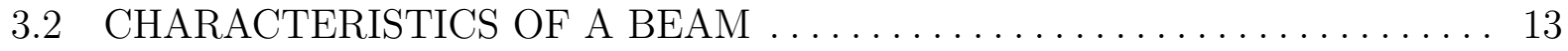

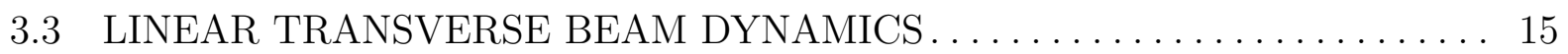

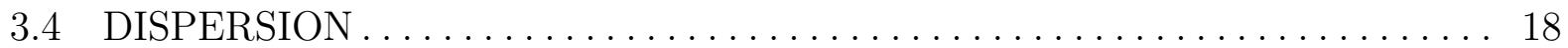

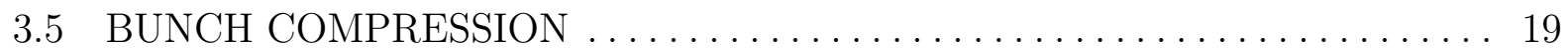

3.6 LONGITUDINAL SYNCHROTRON DYNAMICS $\ldots \ldots \ldots \ldots \ldots \ldots \ldots \ldots .23$

3.7 ADIABATICITY PARAMETER $\ldots \ldots \ldots \ldots \ldots \ldots \ldots \ldots \ldots \ldots \ldots \ldots$

4. TUNABLE BUNCH COMPRESSOR $\ldots \ldots \ldots \ldots \ldots \ldots \ldots \ldots \ldots \ldots \ldots \ldots \ldots \ldots \ldots \ldots \ldots \ldots$

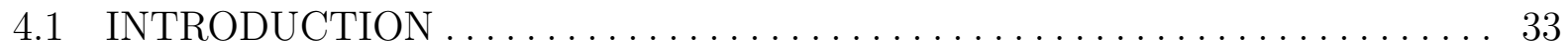

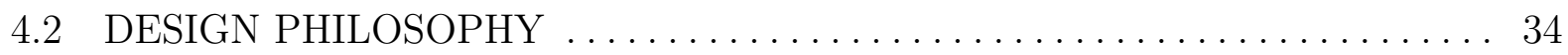

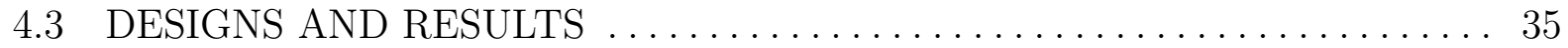

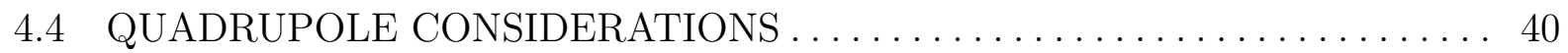

4.5 SECOND ORDER DISPERSION CORRECTION $\ldots \ldots \ldots \ldots \ldots \ldots \ldots \ldots .43$

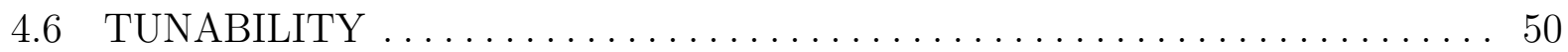

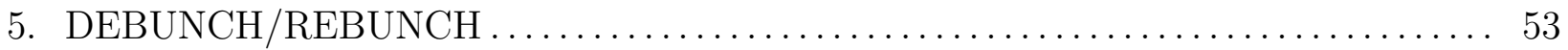

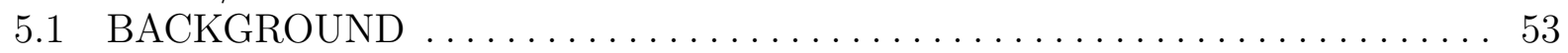

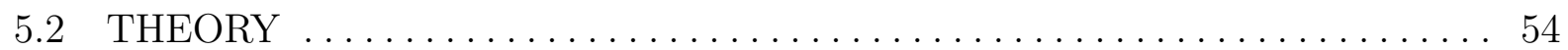

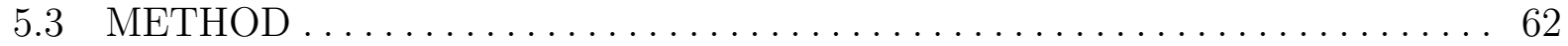

5.4 BROADBAND IMPEDANCE THRESHOLDS $\ldots \ldots \ldots \ldots \ldots \ldots \ldots \ldots \ldots 6$

5.5 NARROW BAND IMPEDANCE $\ldots \ldots \ldots \ldots \ldots \ldots \ldots \ldots \ldots \ldots \ldots \ldots$

6. ADIABATIC BUNCH SPLITTING $\ldots \ldots \ldots \ldots \ldots \ldots \ldots \ldots \ldots \ldots \ldots \ldots \ldots \ldots \ldots$

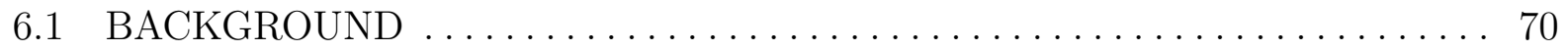

6.2 BUNCH MERGING AND BUNCH SPLITTING $\ldots \ldots \ldots \ldots \ldots \ldots \ldots \ldots \ldots$

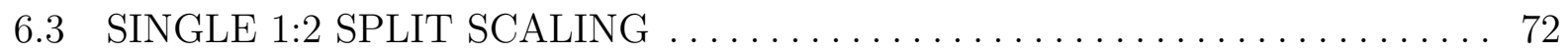

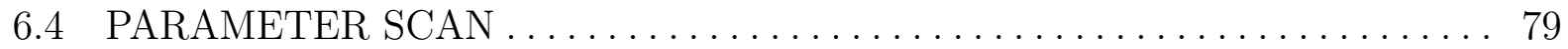


6.5 MULTIPLE $1: 2$ SPLITTING FOR JLEIC $\ldots \ldots \ldots \ldots \ldots \ldots \ldots \ldots \ldots \ldots$

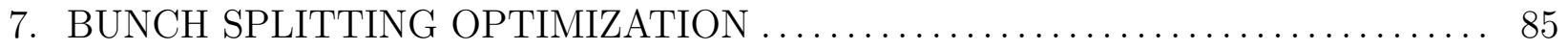

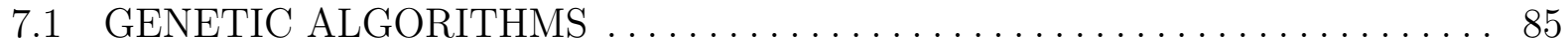

7.2 PARAMETERS FOR THE GENETIC ALGORITHM $\ldots \ldots \ldots \ldots \ldots \ldots$

7.3 RESULTS FROM THE GENETIC ALGORITHM $\ldots \ldots \ldots \ldots \ldots \ldots \ldots$

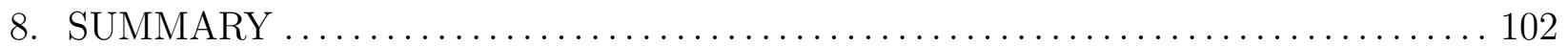

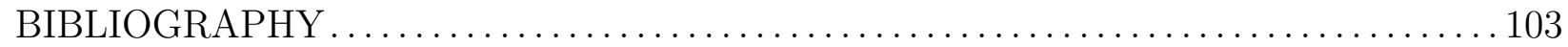

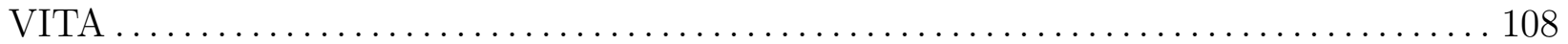




\section{LIST OF TABLES}

Table

Page

$1 \quad$ JLEIC parameters and performance $\ldots \ldots \ldots \ldots \ldots \ldots \ldots \ldots \ldots \ldots$

$2 \quad$ JLEIC Ion Source Requirements $\ldots \ldots \ldots \ldots \ldots \ldots \ldots \ldots \ldots \ldots$

$3 \quad$ Hadron Collider Longitudinal Parameters at Top Energy . . . . . . . . . . . . . . . 10

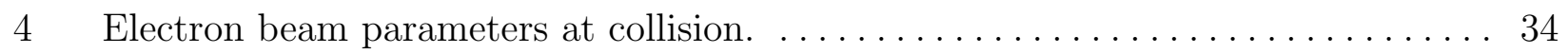

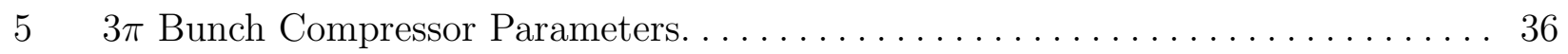

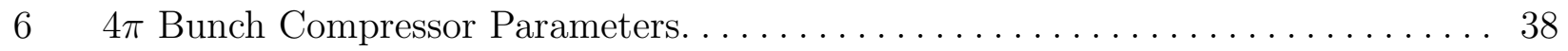

$7 \quad$ Selected data for the quadrupoles used in the design. $\ldots \ldots \ldots \ldots \ldots \ldots \ldots \ldots$

$8 \quad$ RadiaBeam Diamond Quadrupole. . . . . . . . . . . . . . . . . . . . . 40

$9 \quad$ Input beam parameters. $\ldots \ldots \ldots \ldots \ldots \ldots \ldots \ldots \ldots \ldots \ldots \ldots \ldots \ldots$

$10 \quad$ Output beam parameters. $\ldots \ldots \ldots \ldots \ldots \ldots \ldots \ldots \ldots \ldots \ldots \ldots \ldots$

$11 \quad M_{56}$ tunability of the $4 \pi$ compressor. $\ldots \ldots \ldots \ldots \ldots \ldots \ldots \ldots \ldots \ldots \ldots \ldots$

$12 \quad M_{56}$ tunability of the $3 \pi$ compressor. $\ldots \ldots \ldots \ldots \ldots \ldots \ldots \ldots \ldots \ldots \ldots \ldots \ldots \ldots$

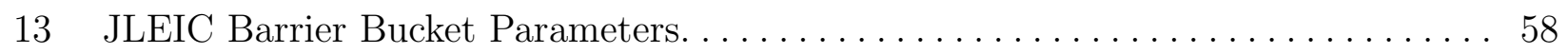

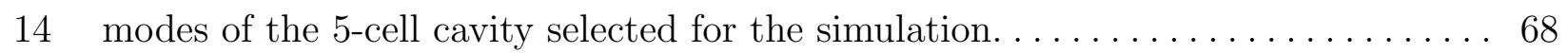

$15 \quad$ JLEIC collider parameters. $\ldots \ldots \ldots \ldots \ldots \ldots \ldots \ldots \ldots \ldots \ldots \ldots \ldots \ldots$

16 Harmonic number and the corresponding amplitude for the whole RF systems used for the complete splitting process. $\ldots \ldots \ldots \ldots \ldots \ldots \ldots \ldots \ldots \ldots \ldots$

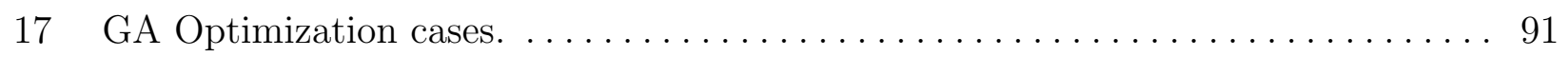

$18 \quad$ RF system parameters used in the GA simulation. . . . . . . . . . . . . . 92 


\section{LIST OF FIGURES}

Figure

Page

$1 \quad$ Schematic diagram of the JLEIC accelerator complex. $\ldots \ldots \ldots \ldots \ldots \ldots \ldots \ldots$

$2 \quad$ Artist's conception of the JLEIC layout on the Jefferson Lab site. . . . . . . . . . 4

$3 \quad$ JLEIC ion linac design. $(* * *$ insert citation $) \ldots \ldots \ldots \ldots \ldots \ldots \ldots \ldots$

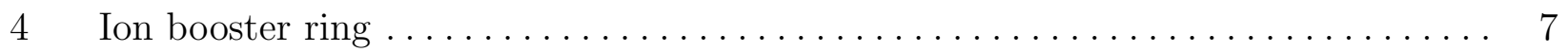

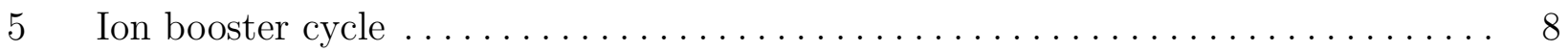

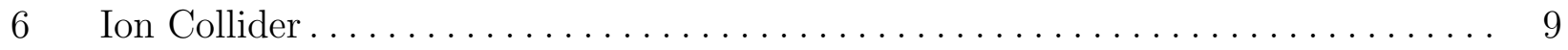

$7 \quad$ Bunch formation before collision $\ldots \ldots \ldots \ldots \ldots \ldots \ldots \ldots \ldots \ldots \ldots$

$8 \quad$ Reference orbit and coordinate system $\ldots \ldots \ldots \ldots \ldots \ldots \ldots \ldots \ldots \ldots$

9 Distribution of particles in horizontal or vertical phase space with a 2D Gaussian distribution, showing projected Gaussian distributions on position and momentum axes and relationships between $\mathrm{rms}(68 \%)$ and 95\% emittance ellipses. . . . . 14

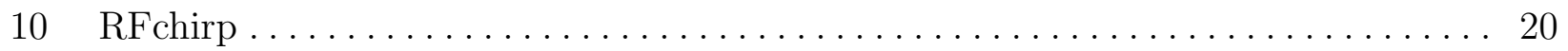

11 A bunch profile before and after going through a bunch compressor. . . . . . . . . 21

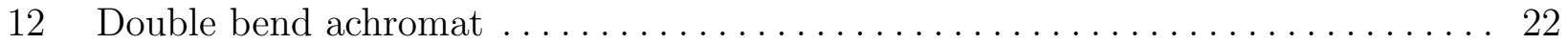

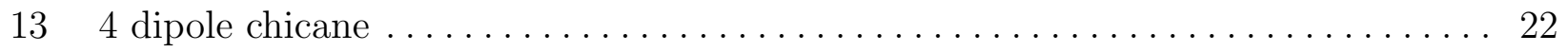

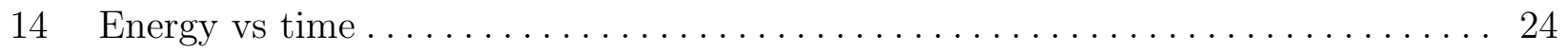

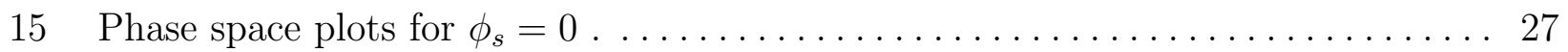

16 Synchrotron period vs the harmonic number for different RF amplitudes. . . . . . . 28

17 RF bucket change with change in the harmonic number and RF amplitude while the $h: V$ ratio kept constant. The area of each bucket are $44.46 \mathrm{eV}$-s, $22.23 \mathrm{eV}$-s

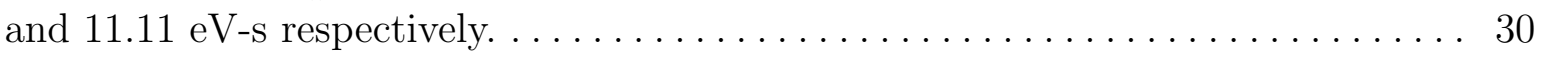

$18 \quad \mathrm{RF}$ wave comparison to the bucket and the bunch inside it. $\ldots \ldots \ldots \ldots \ldots$

19 An iso-adiabatic voltage ramp compared to a linear ramp. With $V_{i}=100 \mathrm{~V}$,

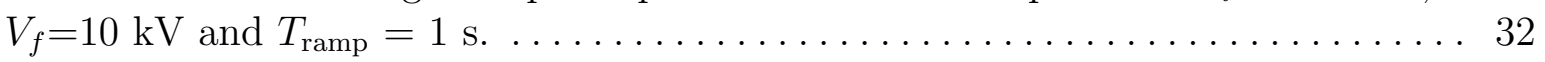


$20 \quad$ Inverse Compton scattering. $\ldots \ldots \ldots \ldots \ldots \ldots \ldots \ldots \ldots \ldots \ldots \ldots \ldots \ldots$

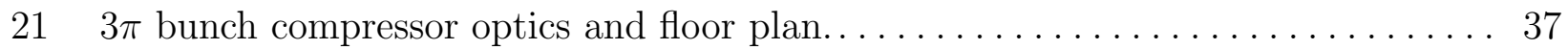

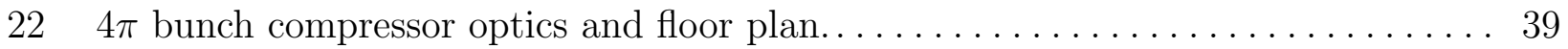

23 Typical fringe field distribution from field measurements and the corresponding

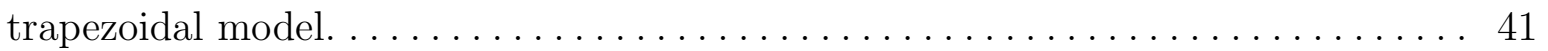

24 Fit of normalized fringe field for Radiabeam diamond quadrupole, consistent with

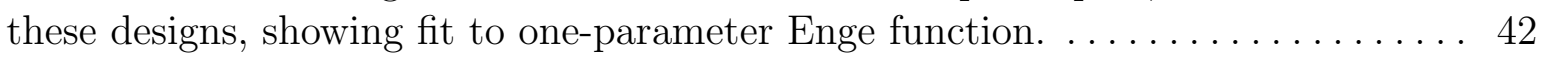

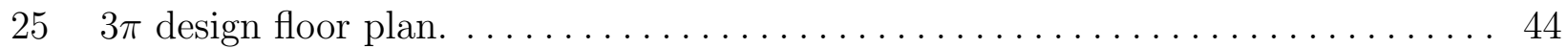

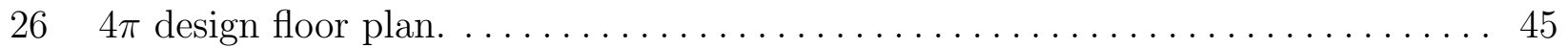

27 Longitudinal phase space (left) and time histogram (right) before compressor (top), after compressor without sextupole correction (center), and after compressor with sextupole correction (bottom) for the $3 \pi$ compressor. $\ldots \ldots \ldots \ldots \ldots 48$

28 Longitudinal phase space (left) and time histogram (right) before compressor (top), after compressor without sextupole correction (center), and after compressor with sextupole correction (bottom) for the $4 \pi$ compressor. $\ldots \ldots \ldots \ldots \ldots 49$

$29 \quad 4 \pi$ beta function and dispersion. $\ldots \ldots \ldots \ldots \ldots \ldots \ldots \ldots \ldots \ldots \ldots \ldots \ldots \ldots \ldots \ldots \ldots$

$30 \quad 3 \pi$ beta function and dispersion. $\ldots \ldots \ldots \ldots \ldots \ldots \ldots \ldots \ldots \ldots \ldots \ldots \ldots \ldots \ldots \ldots \ldots$

31 Barrier bucket form for JLEIC debunch/rebunch bunch formation. Barrier buckets are created in the locations of the two $h=28$ abort gaps, dividing the beam in to two equal sections each containing 13 bunches at $h=28 . \ldots \ldots \ldots \ldots \ldots .55$

32 A phase space distribution of a bunch insider a barrier bucket. $T_{1}$ is half the period of the barrier bucket, $T_{2}$ is the length of time between barrier buckets, and $\hat{T}_{1}$ is the extent to which a given bunch distribution penetrates the barrier bucket

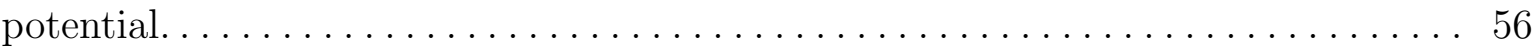

33 Barrier bucket voltage amplitude $v s$ the penetration depth (normalized to T1). For a complete adiabatic debunch the $95 \%$ energy spread is $2 \sigma_{d E}=3.48 \mathrm{MeV}$. Blue line is at the length of one RF bucket of $h=3584 \ldots \ldots \ldots \ldots \ldots \ldots \ldots . \ldots 8$

34 Synchrotron tune plotted against the energy offset of the bunch for different barrier bucket voltages. The dashed lines shows the bucket acceptance for each voltage. $1 \mathrm{kV}$ and $10 \mathrm{kV}$ bucket acceptance lines out of range in the plot. The vertical red line shows the 95\% energy offset for the JLEIC barrier bucket beam. . 59

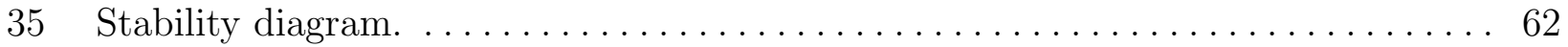


$36 \quad$ RF voltage ramps for a debunch rebunch method. . . . . . . . . . . . . . . 63

37 Required time for an iso adiabatic voltage ramp plotted against the final $\mathrm{RF}$ amplitude for different adiabaticity parameters. $\ldots \ldots \ldots \ldots \ldots \ldots \ldots$

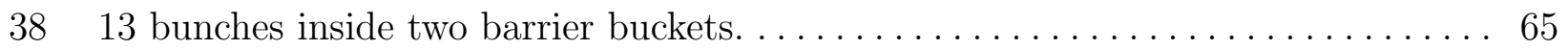

$39 \quad$ Adibatically debunched beam from 13 bi Gaussian bunches. . . . . . . . . . . . . 65

40 Emittance growth (final emittance to initial emittance ratio) for iso-adiabatic

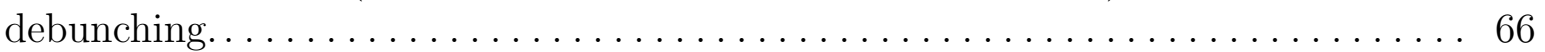

41 Longitudinal impedance vs frequency of the 5-cell $952.6 \mathrm{MHz}$ cavity. . . . . . . . . 67

42 Beam profile at $T=1 \mathrm{~ms}$, for coasting beam instability triggered by a single JLEIC

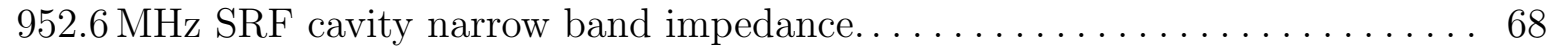

43 Energy spread vs time, for coasting beam instability triggered by a single JLEIC

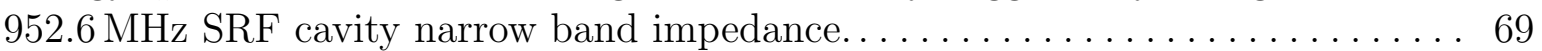

$44 \quad$ 1:3 bunch splitting process in CERN PS and the corresponding RF voltage curves. 72

$45 \quad$ RF amplitude curves for the two RF systems. $\ldots \ldots \ldots \ldots \ldots \ldots \ldots \ldots \ldots \ldots$

46 synchrotron tune of the splitting process. $\ldots \ldots \ldots \ldots \ldots \ldots \ldots \ldots \ldots \ldots \ldots$

47 Synchrotron period plotted against $m$ in log scale where $m=\frac{h V}{h_{0} V_{0}}$ with $h_{0}=28$

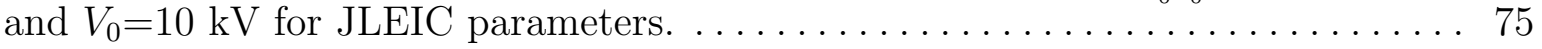

$48 \quad$ Bunch splitting for $T_{\text {split }}=10 T_{\mathrm{s}} . \ldots \ldots \ldots \ldots \ldots \ldots \ldots \ldots \ldots \ldots \ldots \ldots$

$49 \quad$ Bunch splitting for $T_{\text {split }}=10 T_{\mathrm{s}}$ showing the decoherence of the bunches. $\ldots \ldots .77$

$50 \quad$ RF voltages (on a log scale) for the different harmonics used in the complete splitting process as a function of time. $\ldots \ldots \ldots \ldots \ldots \ldots \ldots \ldots \ldots \ldots$

$51 \quad$ Final Bunch formation with $T_{\text {split }}=10 T_{\mathrm{s}}$ for each split. $\ldots \ldots \ldots \ldots \ldots \ldots \ldots$

$52 \quad$ JLEIC $1: 2$ split emittance growth, $T_{\text {split }} / T_{\mathrm{s}}<30 . \ldots \ldots \ldots \ldots \ldots \ldots \ldots \ldots$

$53 \quad$ JLEIC $1: 2$ split emittance growth, $T_{\text {split }} / T_{\mathrm{s}}>30 . \ldots \ldots \ldots \ldots \ldots \ldots \ldots \ldots$

54 Bunch formation with $T_{\text {split }}=10 T_{\mathrm{s}}$ for the initial split with the density heat map. 82

55 Bunch current profile of the splitting process. Each line is plot with $\sim 1 \mathrm{~s}$ time difference. (a) shows the initial split and (b) shows the second split. (c) shows rest of the five remaining splits for one bunch out of the four in (b). . . . . . . 83 
$56 \quad$ Final Bunch formation with $T_{\text {split }}=50 T_{\mathrm{s}}$ for each split. $\ldots \ldots \ldots \ldots \ldots \ldots$

$57 \quad$ Flow chart for the genetic algorithm. $\ldots \ldots \ldots \ldots \ldots \ldots \ldots \ldots \ldots$

58 Diagram of the population advancing to the next generation. $\ldots \ldots \ldots \ldots \ldots$

$59 \quad$ Pareto optimal front. $\ldots \ldots \ldots \ldots \ldots \ldots \ldots \ldots \ldots \ldots \ldots \ldots \ldots \ldots \ldots \ldots$

$60 \quad$ Variables selected for the GA optimization. $\ldots \ldots \ldots \ldots \ldots \ldots \ldots \ldots \ldots \ldots$

61 Pareto optimal curve for linear voltage variation with a bunch to bucket ratio of

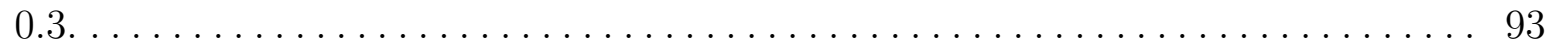

62 Bunch splitting for $T_{\text {split }}=25 T_{\mathrm{s}}$ using the results from|Fig. 61. Emittance growth

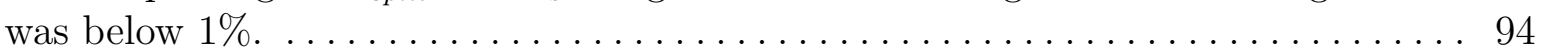

63 Pareto optimal curve for linear voltage variation with a bunch to bucket ratio 0.05. 95

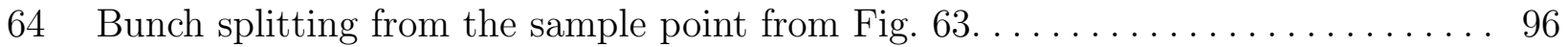

$65 \quad$ A single bunch after the split with $T_{\text {split }}=85 T_{\mathrm{s}} . \ldots \ldots \ldots \ldots \ldots \ldots \ldots$

66 Pareto optimal curve for linear voltage variation with a bunch to bucket ratio 0.3. 98

67 Pareto optimal curve for linear RF voltage amplitude variation with a bunch to

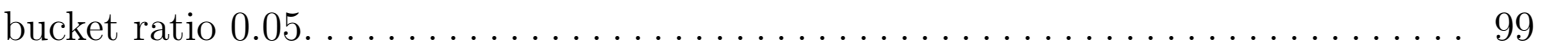

68 Pareto optimal curve for iso-adiabatic voltage variation with a bunch to bucket

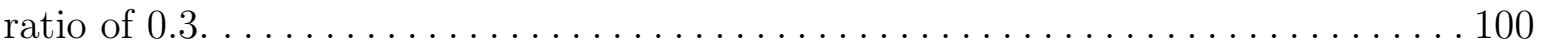

69 Pareto optimal curve for iso-adiabatic voltage variation with a bunch to bucket

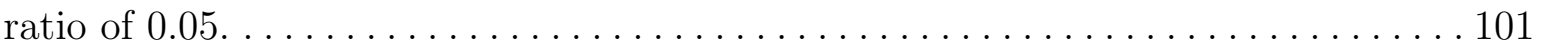




\section{CHAPTER 1}

\section{INTRODUCTION}

The next major accelerator facility likely to be built in the United States is a polarizedbeam electron-ion collider (EIC), a facility designed to support experimental exploration of the structure and interactions of nuclear matter dominated by gluons and the strong force. This facility was strongly supported by the DOE Nuclear Science Advisory Committee 2015 Long Range Plan for Nuclear Science [1]. The underlying nuclear physics motivation and facility requirements were detailed further in a White Paper in 2012 [2].

A team at the Thomas Jefferson National Accelerator Facility in Newport News, Virginia is proposing a design for this EIC. The proposed Jefferson Lab Electron-Ion Collider (JLEIC [3]) requires a large number of short ion bunches to achieve its desired luminosity. This dissertation examines methods to create this large number of short ion bunches in a high energy synchrotron such as the JLEIC ion collider ring.

The first chapter provides an introduction to the overall JLEIC design, focusing mainly on the new ion bunch complex. This is followed by a more detailed look into options of how to solve the "bunch formation problem" - how to produce a large number of short bunches from the ion bunch structure provided by the ion injector design, while limiting longitudinal emittance growth. This section also compares these requirements to similar parameters achieved at other hadron colliders.

The third chapter provides a theoretical background for this dissertation, starting with the basic characteristics of a beam, then reviewing transverse beam dynamics to accommodate the bunch compressor design introduced in a later chapter. The third chapter also reviews the general theory of longitudinal beam dynamics that is used for the bunch formation and RF gymnastics performed in the subsequent chapters.

In addition to addressing ion bunch formation strategies for the JLEIC, this dissertation details a tunable bunch compressor design that was developed for a compact Compton light source proposal [4]. Two options for this tunable bunch compressor are described in chapter four. A brief introduction to Compton scattering is followed by the design philosophy. Two compressors were designed, with net phase advances of $3 \pi$ and $4 \pi$. A section includes details on floor plans, and beam sizes. Since the quadrupoles used in the design are short, the fringe fields of the quadrupoles were taken into consideration. The second part of the chapter 
details the addition of sextupoles for second order corrections to the momentum compaction, followed by the new beam sizes and the floor plans. The last part of this chapter explores the tunability of the system's bunch compression.

To achieve the desired specifications for the JLEIC proposal, different bunch formation strategies are examined. The two main methods under consideration are a debunch/rebunch scheme, and a bunch splitting technique. The fifth chapter of this dissertation explores the debunch/rebunch method that was first considered for ion bunch formation for the JLEIC ion collider ring. The first section describes the method in detail and a historical background. This includes a description and theory of barrier buckets, which are necessary to preserve required abort gaps while debunching the beam. Simulation results of debunching from the longitudinal simulation code ESME are also shown. The next section describes the theoretical background for the stability criteria, which is the Keil-Schnell criterion for pure coasting beams and Keil-Schnell-Boussard criterion for bunched beams, to evaluate whether debunch/rebunch is a feasible approach for ion bunch formation in the JLEIC ion collider ring.

The sixth chapter details the second bunch formation strategy using adiabatic bunch splitting. A brief historical background is provided in the first section, focusing on the CERN PS complex. The second section goes into detail of scaling bunch splitting parameters to be dimensionless so that the process is repeatable, which will be required for JLEIC. It is then followed by a parameter scan of the two dimensionless parameters that have major effects on the emittance growth: the ratio of split time to synchrotron period, and the bunch emittance to bucket area in longitudinal phase space. A seven consecutive split using the results of the parameter scan for one starting bunch and the emittance growth resulting from the optimized process is shown in last section of the chapter.

In the seventh chapter, more variables are added to investigate if they have any effect on reducing the split time while keeping the emittance growth to a minimum. For this purpose a genetic algorithm (GA) was used to reduce the computational resources and time, and to develop optimization parameterization so later bunch split designs can optimally trade off split time and emittance growth. The first section describes the working methodology of the genetic algorithm and the graphical representation of a multi objective solution space with use a Pareto optimal front. Then several cases of RF voltage curves (linear and isoadiabatic) are considered for the GA for multiple values of the dimensionless parameter of bunch emittance to bucket area in longitudinal phase space. 


\section{CHAPTER 2}

\section{JLEIC ION COMPLEX}

Thomas Jefferson National Accelerator Facility (JLab) [5] is one of 17 national laboratories funded by the U. S. Department of Energy. The lab's primary mission is to conduct basic research in nuclear physics using the lab's unique particle accelerator, known as the Continuous Electron Beam Accelerator Facility (CEBAF) [6].

As the construction of the Facility for Rare Isotope Beam (FRIB) at Michigan State University is closing in on completion, the Nuclear Science Advisory Committee (NSAC) of the US Department of Energy and the National Science Foundation released their 2015 annual report [1] that detailed the requirements for a next-generation accelerator facility for the USA. The white paper [2] requirements for this facility are:

- Highly polarized (70\%) electron and nucleon beams

- Ion beams (polarized protons, deuterium, and helium-3, and un-polarized heavy ions up to lead)

- Variable particle energies for variable center-of-mass collision energies:

- From $3 \mathrm{GeV}$ to $11 \mathrm{GeV}$ for electrons

- From 20 to $\sim 100 \mathrm{GeV}$ for protons

- Up to $40 \mathrm{GeV}$ per nucleon for ions

- High collision luminosity in the range of mid $10^{33} \mathrm{~cm}^{-2} \mathrm{~s}^{-1}$ to above $10^{34} \mathrm{~cm}^{-2} \mathrm{~s}^{-1}$ per interaction point

- Possibility of having more than one interaction region

The proposed Jefferson Lab Electron-Ion Collider (JLEIC, formerly MEIC) is designed to meet these criteria. A recent iteration of the facility's technical design is detailed in [2] and [7]. The JLEIC design achieves high luminosity in its design center of mass (CM) energy range of 15-65 GeV with large collision frequency, short bunches, and modest bunch charges. Though this strategy has been successful at B-factories, the JLEIC design requires 
substantially shorter hadron bunch lengths $\sigma_{\mathrm{z}}$ at a higher RF frequency $f_{\mathrm{RF}}$ than any previous collider.

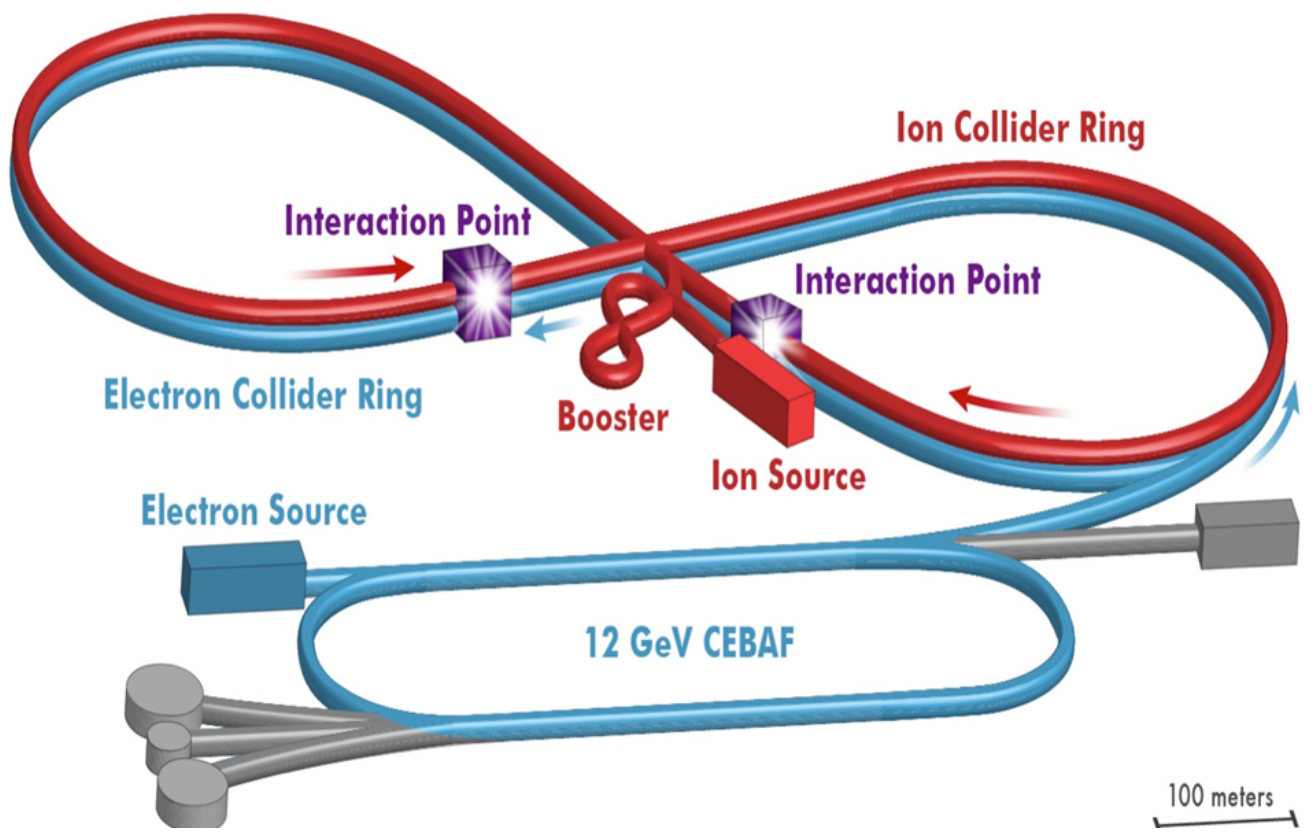

FIG. 1: Schematic diagram of the JLEIC accelerator complex.

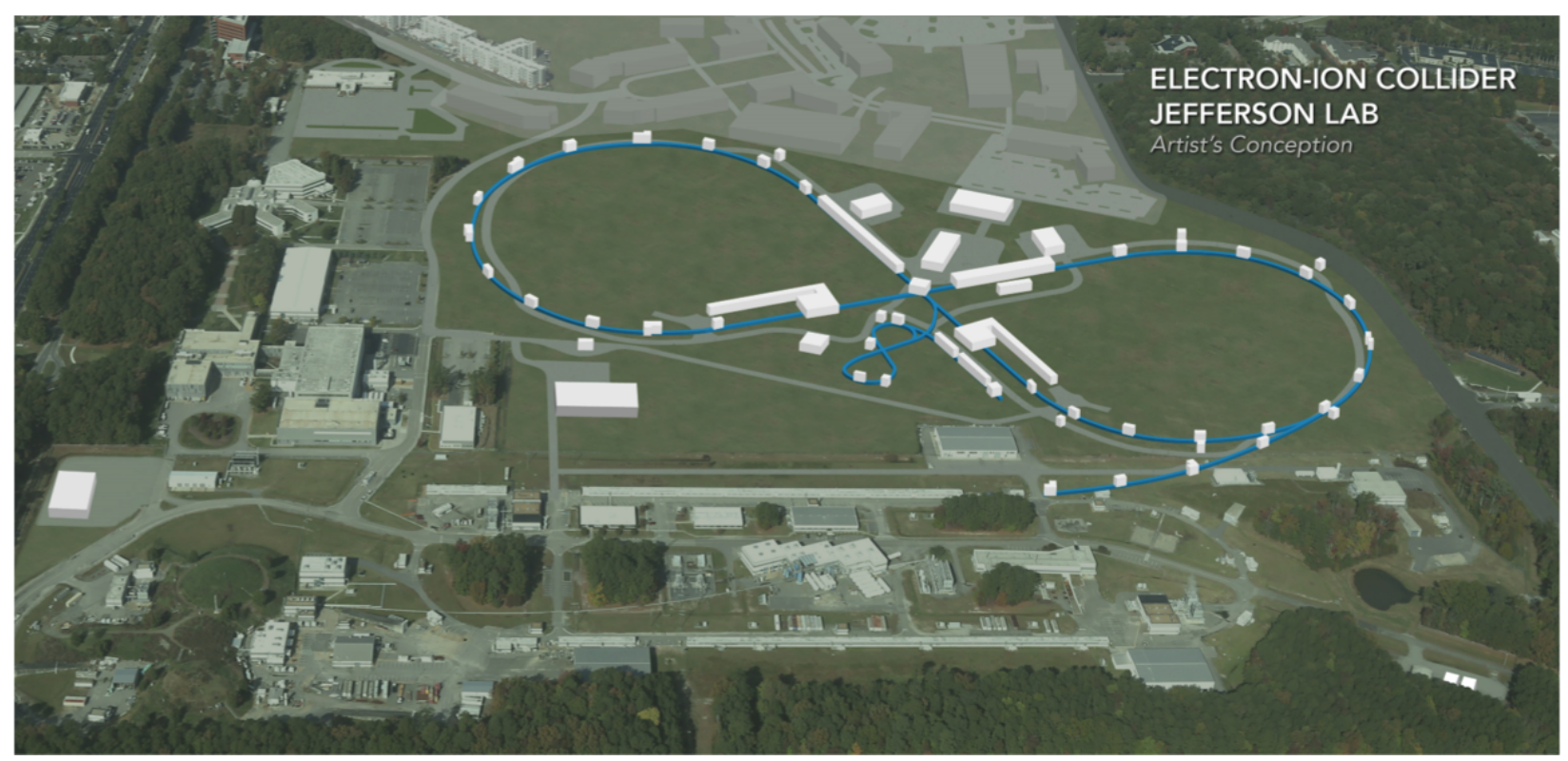

FIG. 2: Artist's conception of the JLEIC layout on the Jefferson Lab site.

A schematic diagram of the JLEIC accelerator complex is shown in Fig. 1, while an 
artist's conception of a bird's-eye view of the collider on the JLab site is show in Fig. 2. The figure-8 topology is a design feature to ensure longer polarization lifetime of polarized hadron beams. JLEIC design parameters are detailed in Table 1, where the collision frequency and bunch length for protons are the main parameters relevant to this dissertation.

TABLE 1: JLEIC parameters and performance

\begin{tabular}{|c|c|c|c|c|c|c|c|}
\hline \multirow{3}{*}{$\begin{array}{l}\text { Parameter } \\
\text { CM energy }\end{array}$} & \multirow{3}{*}{$\begin{array}{l}\text { Units } \\
\mathrm{GeV}\end{array}$} & \multicolumn{2}{|c|}{ low } & \multicolumn{2}{|c|}{ medium } & \multicolumn{2}{|c|}{ high } \\
\hline & & \multicolumn{2}{|c|}{21.9} & \multicolumn{2}{|c|}{44.7} & \multicolumn{2}{|c|}{63.3} \\
\hline & & $\mathrm{p}$ & e & $\mathrm{p}$ & e & $\mathrm{p}$ & e \\
\hline Beam energy & $\mathrm{GeV}$ & 40 & 3 & 100 & 5 & 100 & 10 \\
\hline Collision Frequency & $\mathrm{MHz}$ & \multicolumn{2}{|c|}{476} & \multicolumn{2}{|c|}{476} & \multicolumn{2}{|c|}{119} \\
\hline Particle per bunch $\left(10^{10}\right)$ & & 0.98 & 3.7 & 0.98 & 3.7 & 3.9 & 3.7 \\
\hline Beam current & $\mathrm{A}$ & 0.75 & 2.8 & 0.75 & 2.8 & 0.75 & 0.71 \\
\hline Polarization & $\%$ & 80 & 80 & 80 & 80 & 80 & 70 \\
\hline Bunch length (RMS) & $\mathrm{cm}$ & 3 & 1 & 1 & 1 & 2.2 & 1 \\
\hline Norm. emittance, horiz./vert. & $\mu \mathrm{m}$ & $0.3 / 0.3$ & $24 / 24$ & $0.5 / 0.1$ & $54 / 10.8$ & $0.9 / 0.18$ & $432 / 86.4$ \\
\hline Horizontal \& vertical $\beta^{*}$ & $\mathrm{~cm}$ & $8 / 8$ & $13.5 / 13.5$ & $6 / 1.2$ & $5.1 / 1$ & $10.5 / 2.1$ & $4 / 0.8$ \\
\hline Vert. beam-beam parameter & & 0.015 & 0.092 & 0.015 & 0.068 & 0.008 & 0.034 \\
\hline Laslett tune-shift & & 0.06 & $7 \times 10^{-4}$ & 0.055 & $6 \times 10^{-4}$ & 0.056 & $7 \times 10^{-5}$ \\
\hline Detector space, up/down & $\mathrm{m}$ & $3.6 / 7$ & $3.2 / 3$ & $3.6 / 7$ & $3.2 / 3$ & $3.6 / 7$ & $3.2 / 3$ \\
\hline Hourglass reduction & & \multicolumn{2}{|r|}{1} & \multicolumn{2}{|c|}{0.87} & \multicolumn{2}{|c|}{0.75} \\
\hline Luminosity/IP, w/HG, $\left(\times 10^{33}\right)$ & $\mathrm{cm}^{-2} \mathrm{~s}^{-1}$ & \multicolumn{2}{|c|}{2.5} & \multicolumn{2}{|c|}{21.4} & \multicolumn{2}{|c|}{5.9} \\
\hline
\end{tabular}

\subsection{JLEIC ION INJECTOR COMPLEX}

Bunch formation of the JLEIC ion beams starts with the ion injector complex. This complex consists of ion sources, a linac, and a smaller figure- 8 booster accelerator. These provide the ion beam bunches (and bunch structure) to the ion collider ring that is described in section 2.2.

\subsubsection{ION SOURCES}

The required ion species will be produced by two types of ion sources. An Atomic Beam Polarized Ion Source (ABPIS) will provide polarized or non-polarized light ions. An Electron 
Beam Ion Source (EBIS) will provide un-polarized heavy ions (up to $\mathrm{Pb}$ ).

The requirements for these ion sources are included in Table 2. Note that typical pulse lengths from these ion sources are tens to hundreds of $\mu \mathrm{s}$, much longer than the short bunches required at collision, and the average current is modest. These long bunches are shaped into short bunches in the linac, and accumulated in the booster to higher current, before being accelerated and transferred to the ion collider ring for further bunch formation.

TABLE 2: JLEIC Ion Source Requirements

\begin{tabular}{llcc}
\hline \hline & & Polarized Light Ions & Unpolarized Heavy Ions \\
\hline Parameter & Units & Value & Value \\
\hline Ion species & {$[-]$} & $\mathrm{H}^{-}$ & ${ }^{208} \mathrm{~Pb}^{30+}$ \\
Pulse current & {$[\mathrm{mA}]$} & 2.0 & 1.3 \\
Pulse length & {$[\mathrm{ms}]$} & 0.5 & 0.01 \\
Charge per pulse & {$[\mu \mathrm{C}]$} & 1.0 & 0.013 \\
Ions per pulse & {$\left[10^{10}\right]$} & 624 & 0.27 \\
Repetition rate & {$[\mathrm{Hz}]$} & $5-10$ & $5-10$ \\
Emittance (norm. $)$ & {$[\pi \mathrm{mm}-\mathrm{mrad}]$} & 2.0 & 0.5 \\
Polarization & {$[-]$} & 0.9 & 0 \\
\hline \hline
\end{tabular}

\subsubsection{ION LINAC}

The ion linac will consist of both warm and cold RF systems, and will accelerate the ion pulses being produced by the source to $280 \mathrm{MeV}$ for protons and $\sim 100 \mathrm{MeV} / \mathrm{u}$ for $\mathrm{Pb}$ ions. Electron stripping will be done according to the type of the ion species. The current design of the linac is effective in accelerating a wide variety of ions from $\mathrm{H}^{-}(280 \mathrm{MeV})$ to lead ion ${ }^{208} \mathrm{~Pb}^{67+}(100 \mathrm{MeV} / \mathrm{u})$. Efficient acceleration of heavy ions (to $100 \mathrm{MeV} / \mathrm{u}$ ) requires a stripper in the linac with an optimum stripping energy of $13 \mathrm{MeV} / \mathrm{u}$. (*** insert citation here) The warm RF section of the linac will accelerate all ion species up to $4.8 \mathrm{MeV} / \mathrm{u}$, and is highly effective for pulsed machines. The superconducting RF part of the linac consist of quarter wave resonator (QWR) and half wave resonator (HWR) cavities, which will operate on the velocity range of $0.1 c$ to $0.5 c$. 


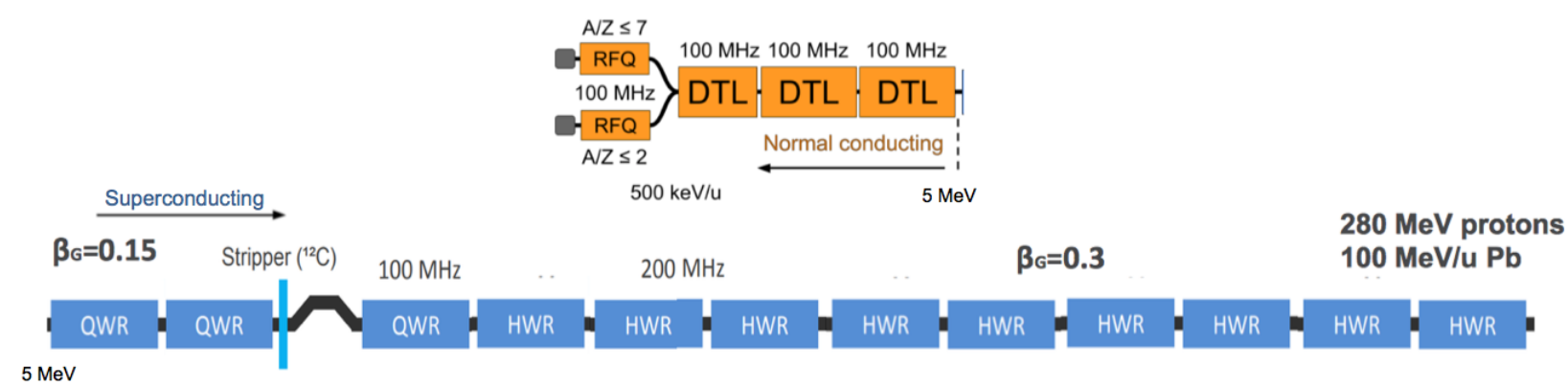

FIG. 3: JLEIC ion linac design. (*** insert citation)

\subsubsection{BOOSTER RING}

The JLEIC booster ring will accelerate the beam up to $8 \mathrm{GeV}$ (for protons; the corresponding energies for ions scaled by the mass to charge ratio to preserve magnetic rigidity $B \rho=\frac{p}{q}$ ). The current design of the booster ring is figure 8 shaped and features two $255^{\circ}$ arcs connected by two dispersion free straight sections that result in $313.5 \mathrm{~m}$ circumference. The achromatic arcs are design to avoid any transition crossing for all ion species during the acceleration stage.

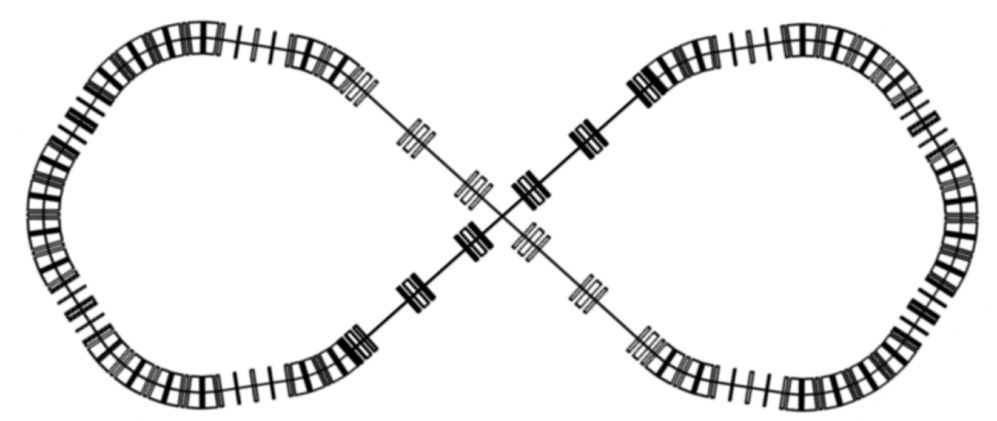

FIG. 4: Ion booster ring

Booster bunch formation in the ion collider ring is as shown in Fig. 5. Ion beams are injected to the booster from the linac (A). This coasting beam is then adiabatically captured in to a single bucket that is approximately $200 \mathrm{~m}$ in length(B). This bunch is then accelerated to an intermediate energy ( $\sim 2 \mathrm{GeV}$ for protons), where $\mathrm{DC}$ cooling is performed before the beam ramped up to $8 \mathrm{GeV}$ for protons $(\mathrm{C})$. Then the bunch length is compressed to $56 \mathrm{~m}$ and split in to two bunches with each having a length of $28 \mathrm{~m}$ (in a $40 \mathrm{~m}$ bucket) (D). Bucket 
to bucket transfer of the two bunches in to the main ion collider ring is then performed. This is done 12 more times to get 26 total bunches into the ion collider ring.

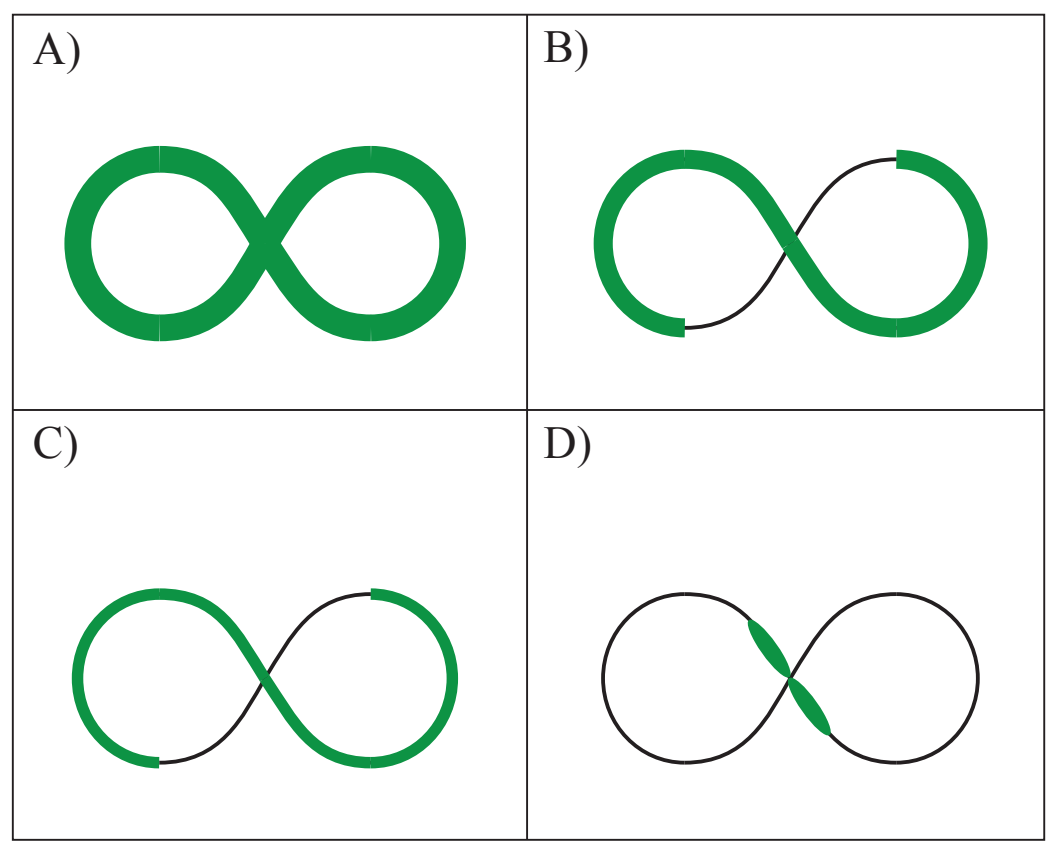

FIG. 5: Ion booster cycle

\subsection{JLEIC ION COLLIDER RING}

Currently there are several bunch formation schemes that are being considered for the final bunch formation before collision in the ion collider ring. The main proposed method is to do a bucket to bucket transfer of pairs of long bunches to the collider ring from the booster with 13 repetitions for 26 bunches total as seen in Fig. 6. 


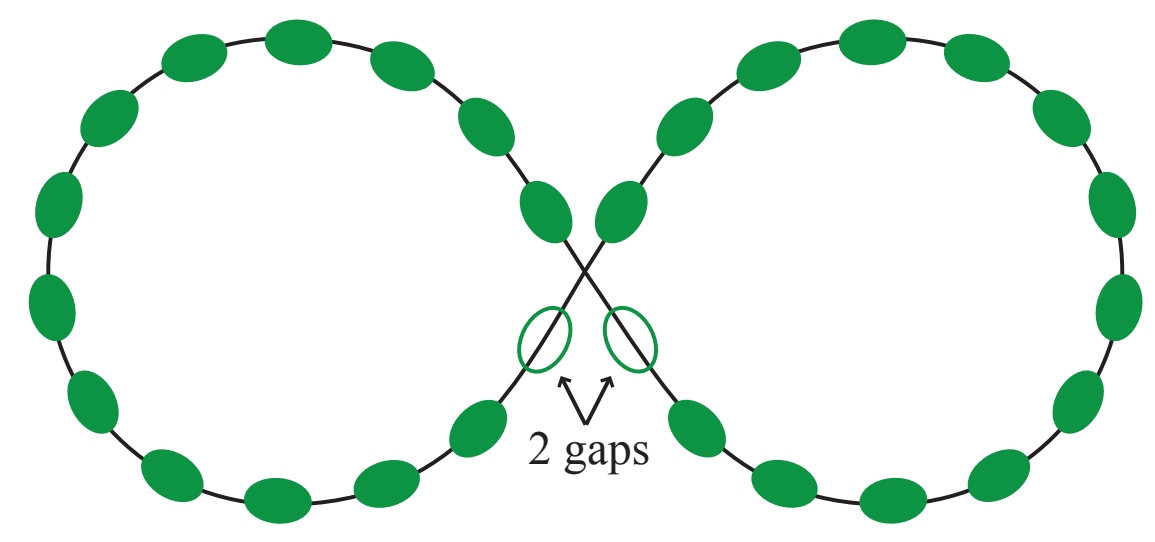

FIG. 6: Ion Collider

With the collider having 26 bunches and 2 gaps with lengths equal to the bucket length the energy is ramped up to the final collision energy. Then binary splits are done 7 times to reach the final number of bunches required, which is 3584 .

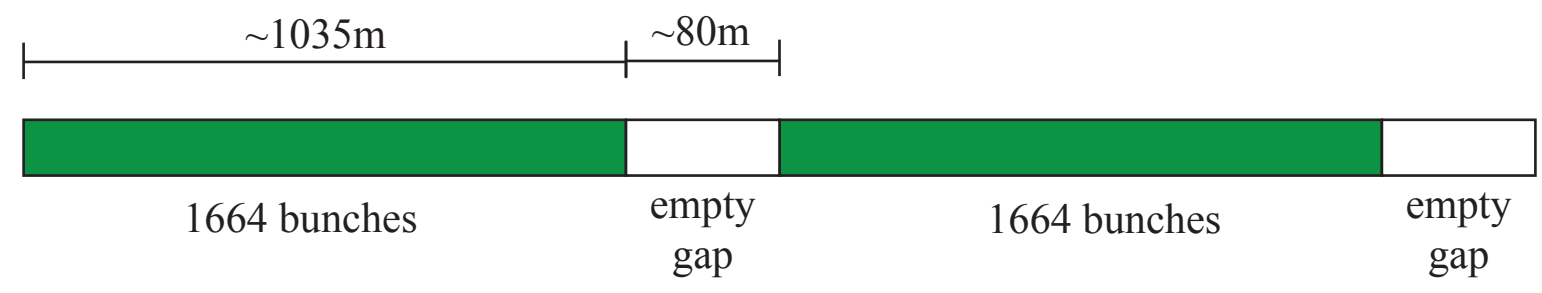

FIG. 7: Bunch formation before collision

The JLEIC design achieves high luminosities in its design CM energy range of 15-65 GeV with high collision frequency, short bunches, and modest bunch intensity. The JLEIC design requires substantially shorter hadron rms bunch lengths $\sigma_{\mathrm{z}}$ in higher $\mathrm{RF}$ frequency $f_{\mathrm{RF}}$ than any previous hadron collider. These differences are itemized in Table 3, RHIC parameters are for re-bucketed $\mathrm{Au}$ ions at $100 \mathrm{GeV} / \mathrm{u}$. 
TABLE 3: Hadron Collider Longitudinal Parameters at Top Energy

\begin{tabular}{llcccc}
\hline \hline Parameter & Units & $\begin{array}{c}\text { JLEIC } \\
(\text { design })\end{array}$ & $\begin{array}{c}\text { RHIC } \\
\text { (achieved) }\end{array}$ & $\begin{array}{c}\text { HERA-p } \\
\text { (achieved) }\end{array}$ & $\begin{array}{c}\text { LHC } \\
\text { (achieved) }\end{array}$ \\
\hline$f_{\mathrm{RF}}$ & {$[\mathrm{MHz}]$} & 953 & 196 & 208 & 400 \\
$\sigma_{z}$ & {$[\mathrm{~cm}]$} & 1 & 25 & 25 & 7.6 \\
$\mathrm{p} /$ bunch & {$\left[10^{10}\right]$} & $0.7-1.0$ & 24 & 7.5 & 11 \\
Energy & {$[\mathrm{GeV}]$} & 100 & 250 & 920 & 7000 \\
$\lambda_{\mathrm{RF}}$ & {$[\mathrm{cm}]$} & 31 & 153 & 144 & 75 \\
$V_{\mathrm{RF}}$ & {$[\mathrm{MV}]$} & 19 & 6 & 2.4 & 12 \\
Circumference & {$[\mathrm{km}]$} & 2.23 & 3.83 & 6.34 & 26.6 \\
$\gamma_{\mathrm{T}}$ & - & 12.5 & 22.9 & 27.4 & 55.7 \\
$\eta$ & {$\left[\times 10^{-4}\right]$} & 64.5 & 18.2 & 13.3 & 3.22 \\
Bucket area & {$[\mathrm{eV}-\mathrm{s}]$} & 0.22 & 1.5 & 3.0 & 7.9 \\
Bucket height $d p / p$ & {$\left[\times 10^{-3}\right]$} & 1.66 & 2.34 & 0.53 & 0.35 \\
\hline \hline
\end{tabular}




\section{CHAPTER 3}

\section{THEORY}

The position of a charged particle in space can be represented by two inertial frames of reference $S$ and $S^{\prime}$ with the coordinates for a single point being defined by $(x, y, z, c t)$ and $(X, Y, Z, c T)$ respectively. One frame is at rest with respect to the lab while the other moves with the reference particle (also know as the synchronous particle) in the $\hat{z}$ direction. The reference particle in this frame is at zero in all coordinates. The two frames are related by the Lorentz transformation:

$$
\begin{array}{r}
x=X \\
y=Y \\
z=\gamma(Z-\beta c t) \\
c t=\gamma(c T-\beta z) .
\end{array}
$$

Where $\beta \equiv v / c$ is the relativistic speed and the relativistic factor $\gamma$ is defined by

$$
\gamma \equiv \frac{1}{\sqrt{1-\beta^{2}}}
$$

For $\beta \ll 1$ non-relativistic approximations close to classical mechanics are used to track equations of motion. For purposes of all chapters except Chapter 3 in this dissertation, particle motion is quite relativistic $(\beta \approx 1, \gamma \gg 1)[8,9]$.

\subsection{COORDINATE SYSTEMS AND PHASE SPACE COORDINATES}

For an accelerator that is designed to keep a particle of charge $q$ in a circular orbit of radius $\rho$, we define a coordinate system $(x, y, z)$ with respect to the location of a reference particle of momentum $p_{0}$ as shown in Fig. 8 . 


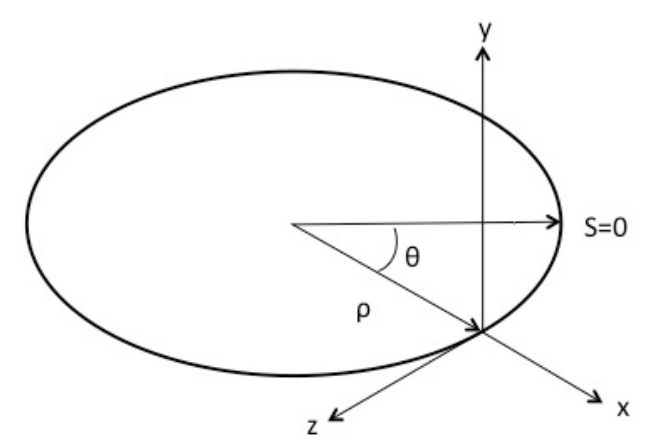

FIG. 8: Reference orbit and coordinate system

The circle represent the design orbit which is the path of the design particle. It is the ideal trajectory for all the test particles to take. In the lab frame, the position of the design particle can be represented by the parameter $\theta$. But for variations of test particles that defer from the ideal trajectory it is important to have a frame $(x, y, z)$ which moves with the design particle. With that any particle can be represented by coordinates $\left(x, p_{x}, y, p_{y}, z, p_{z}\right)$ where $\left(p_{x}, p_{y}, p_{z}\right)$ are corresponding momentum components. The transverse motion is described by the $\left(x, p_{x}, y, p_{y}\right)$ coordinates, and longitudinal motion by $\left(z, p_{z}\right)$ coordinates.

Momentum coordinates are commonly scaled to the reference or design momentum $p_{0}$. This defines new dimensionless momentum coordinates: $x^{\prime} \equiv p_{x} / p_{0}, y^{\prime} \equiv p_{y} / p_{0}$, and $\delta \equiv$ $\left(p-p_{0}\right) / p_{0} . \delta$ is often called the fractional momentum offset of a particle relative to design. $x^{\prime}$ and $y^{\prime}$ are small angles (typically mrad or less), so the paraxial approximation applies and $p_{z} \approx p$ so $\delta \approx\left(p_{z}-p_{0}\right) / p_{0}$, where $p_{0}$ is the momentum of the synchronous or reference particle. Position vs. momentum, or angle, can be plotted for each dimension in a phase space diagram. See, for example, Fig. 9 which shows typical linear phase space for $\left(x, x^{\prime}\right)$ or $\left(y, y^{\prime}\right)$.

Different accelerator codes define the $z$ coordinate with different, related variables. For example ESME defines the $z$ coordinate with RF phase scaled with respect to the harmonic number $h$ of the primary active RF system. ELEGANT [10] defines the $z$ coordinate by the time coordinate. It is important to note that the $t$ and $z$ have opposite signs. On the time axis the design particle is always at zero. So a test particle that is ahead of the design particle (positive $z$ ) will arrive earlier and register as a negative sign in the time axis. Likewise the particles that are behind will have a negative $z$ but a positive time.

Later in this dissertation, we also use the longitudinal dynamics simulation code ESME, 
which uses longitudinal coordinates of $(\theta, d E) . \theta$ is defined as the angular coordinate and it is related to the time coordinate by a factor $360 / T_{\text {rev }}$ where $T_{\text {rev }}$ is the revolution time period. $d E$ is related to the fractional momentum spread or offset by

$$
\frac{d E}{E}=\frac{1}{\beta^{2}} \frac{d p}{p}
$$

\subsection{CHARACTERISTICS OF A BEAM}

For linear motion, the distribution of the beam in 2D phase space can be circumscribed by an ellipse of the form

$$
\epsilon_{x}=\gamma_{x} x^{2}+2 \alpha_{x} x x^{\prime}+\beta_{x} x^{2}
$$

where $\epsilon$ is called the emittance, and $\pi \epsilon$ gives the area enclosed by the ellipse. Note that there are horizontal, vertical, and longitudinal emittances. There are several different conventions used in the community to specify the fraction of the beam inside this ellipse. For example in proton accelerators, an ellipse which contains $90 \%$ of the particles is typically considered as the $90 \%$ emittance, while the electron beam community commonly uses the rms emittance defined by

$$
\epsilon_{\mathrm{rms}}^{2} \equiv<x^{2}><x^{\prime 2}>-<x x^{\prime}>^{2} .
$$




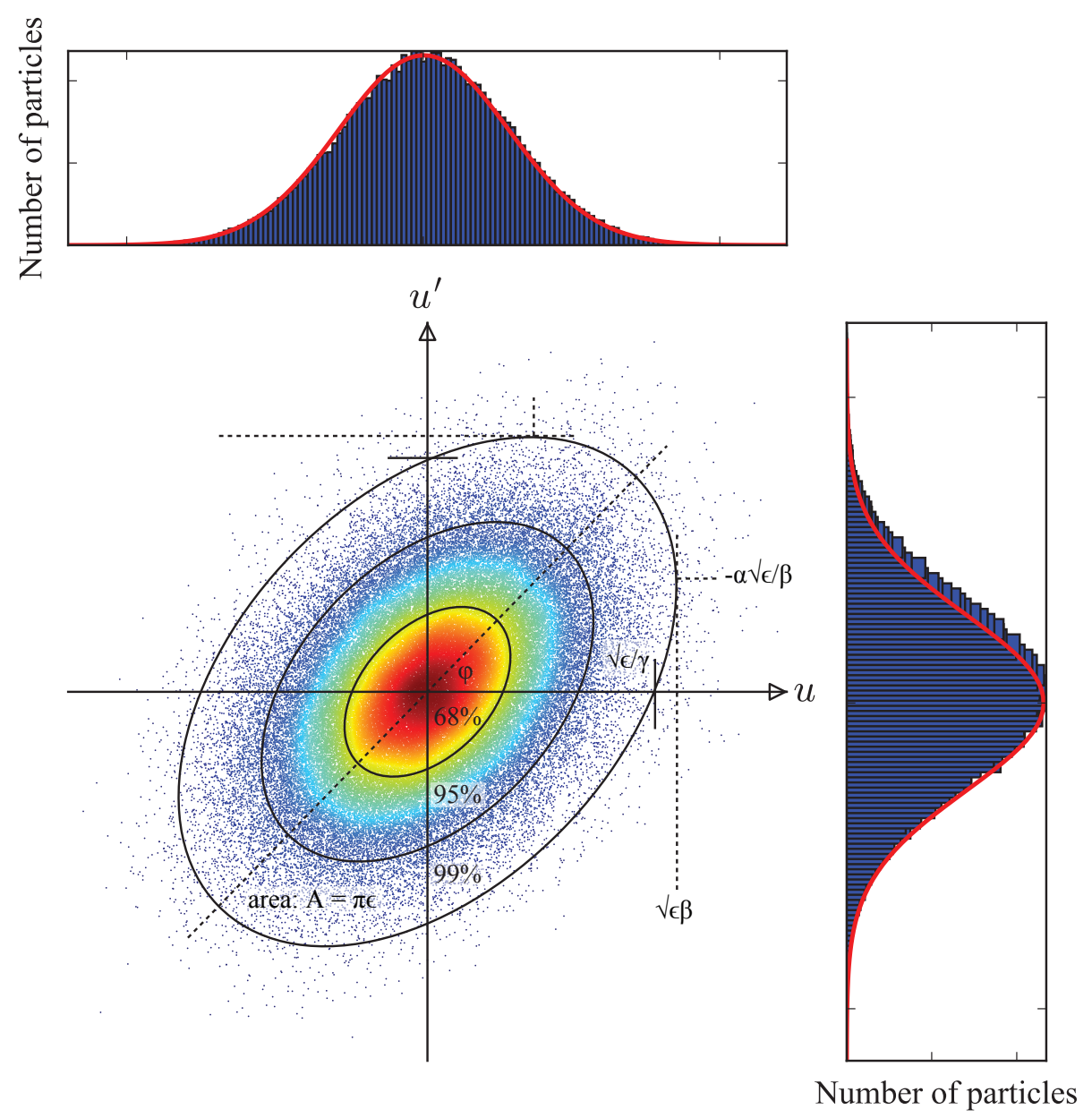

FIG. 9: Distribution of particles in horizontal or vertical phase space with a 2D Gaussian distribution, showing projected Gaussian distributions on position and momentum axes and relationships between rms $(68 \%)$ and $95 \%$ emittance ellipses.

The Courant-Snyder parameters [11] (or the Twiss parameters) $(\beta, \alpha, \gamma)$ are related to the beam distribution by,

$$
\begin{array}{r}
\beta_{\mathrm{x}}=\frac{\sigma_{x}^{2}}{\epsilon_{\mathrm{rms}, \mathrm{x}}} \\
\alpha_{\mathrm{x}}=-\frac{\sigma_{x x^{\prime}}}{\epsilon_{\mathrm{rms}, \mathrm{x}}} \\
\gamma_{\mathrm{x}}=\frac{\sigma_{x^{\prime}}^{2}}{\epsilon_{\mathrm{rms}, \mathrm{x}}}
\end{array}
$$

where $\sigma_{\mathrm{x}}$ and $\sigma_{\mathrm{x}^{\prime}}$ are the rms moments of the beam distribution, and $\sigma_{\mathrm{xx}^{\prime}}$ is the crosscorrelation rms moment. Luminosity is defined as the collision rate per unit cross section, a 
beam interaction (with another beam or a target) will generate. The number is a measurement of the collected data, so a higher number will relate to a high quantity of data. For collisions of two beams with equal rms beam sizes $\sigma_{x, y}$, the luminosity is given by

$$
L=f_{0} N_{\mathrm{b}} \frac{N_{+} N_{-}}{4 \pi \sigma_{\mathrm{x}} \sigma_{\mathrm{y}}}
$$

where $N_{+}$and $N_{-}$are number of particles per bunch in the two colliding beams, $N_{\mathrm{b}}$ is the (equal) number of bunches in each beam, and $f_{0}$ is the revolution frequency.

\subsection{LINEAR TRANSVERSE BEAM DYNAMICS}

Transfer matrices are the mathematical representations of linear optical elements (including drift spaces). Each element has its own unique matrix, and by multiplying these together, one single matrix can be gained that represents the whole lattice which would map a particle from starting point $s=0$ to a new point $s$ further through the accelerator. Using the coordinates from the previous section,

$$
\left[\begin{array}{c}
x \\
x^{\prime} \\
y \\
y^{\prime} \\
z \\
\delta
\end{array}\right]=M(s)\left[\begin{array}{l}
x_{0} \\
x_{0}^{\prime} \\
y_{0} \\
y_{0}^{\prime} \\
z_{0} \\
\delta_{0}
\end{array}\right] .
$$

The linear motion in a periodic system is governed by the second order differential equations of motion known as the Hill's Equations [12]. In two dimension (with the assumption that there are no vertical bends) this is written as

$$
\begin{aligned}
x^{\prime \prime}(s)+\left(\frac{1}{\rho^{2}(s)}-k(s)\right) x(s) & =\frac{1}{\rho(s)} \delta \\
y^{\prime \prime}(s)+k(s) y(s) & =0
\end{aligned}
$$

where $k(s)$ is the quadrupole strength, and $\rho(s)$ is the bending radius, at a given longitudinal position $s$ in the beam pipe. We are considering the case for a monochromatic beam where we can let $\delta \rightarrow 0$. Letting $K_{x}(s)=\left(1 / \rho^{2}(s)-k(s)\right)$ and $K_{y}(s)=k(s)$, the Hill's equations are reduced to those of a simple harmonic oscillator with periodic focusing:

$$
\begin{aligned}
& x^{\prime \prime}(s)+K_{\mathrm{x}}(s) x(s)=0 \\
& y^{\prime \prime}(s)+K_{\mathrm{y}}(s) y(s)=0 .
\end{aligned}
$$


Solutions to these equations can be written in a matrix form with cosine-like and sine-like elements:

$$
\left[\begin{array}{c}
x(s) \\
x^{\prime}(s) \\
y(s) \\
y^{\prime}(s)
\end{array}\right]=\left[\begin{array}{cccc}
C_{\mathrm{x}}(s) & S_{\mathrm{x}}(s) & 0 & 0 \\
C_{\mathrm{x}^{\prime}}(s) & S_{\mathrm{x}^{\prime}}(s) & 0 & 0 \\
0 & 0 & C_{\mathrm{y}}(s) & S_{\mathrm{y}}(s) \\
0 & 0 & C_{\mathrm{y}^{\prime}}(s) & S_{\mathrm{y}^{\prime}}(s)
\end{array}\right]=\left[\begin{array}{c}
x_{0} \\
x_{0}^{\prime} \\
y_{0} \\
y_{0}^{\prime}
\end{array}\right]
$$

\subsubsection{TRANSPORT MATRICES}

A drift is a section of the beam pipe with no electromagnetic fields, so particles experience no external forces to change their linear trajectories. In the highly relativistic approximation, the longitudinal phase coordinates are left unchanged throughout a drift while the transverse phase space is changed. The Hill's equations corresponding to a drift is

$$
\begin{aligned}
& x^{\prime \prime}(s)=0 \\
& y^{\prime \prime}(s)=0 .
\end{aligned}
$$

The solutions for traversing a drift of length $l$ are then

$$
\begin{aligned}
x(l) & =x_{0}+l x_{0}^{\prime} \\
x^{\prime}(l) & =x_{0}^{\prime} \\
y(l) & =y_{0}+l y_{0}^{\prime} \\
y^{\prime}(l) & =y_{0}^{\prime}
\end{aligned}
$$

which can be represented in a matrix form,

$$
M_{\text {drift }}=\left[\begin{array}{llll}
1 & l & 0 & 0 \\
0 & 1 & 0 & 0 \\
0 & 0 & 1 & l \\
0 & 0 & 0 & 1
\end{array}\right]
$$

Quadrupoles are magnets that add $s$-dependent linear focusing to an accelerator. Keeping these linear focusing strengths in Hill's equations gives

$$
\begin{aligned}
& x^{\prime \prime}(s)+k(s) x(s)=0 \\
& y^{\prime \prime}(s)-k(s) y(s)=0 .
\end{aligned}
$$


Solutions traversing a quadrupole of length $l$ are given by

$$
\begin{aligned}
& x(l)=x_{0} \cosh \sqrt{k} l+\frac{x_{0}^{\prime}}{\sqrt{k}} \sinh \sqrt{k} l \\
& x^{\prime}(l)=x_{0} \sqrt{k} \sinh \sqrt{k} l+x_{0}^{\prime} \cosh \sqrt{k} l \\
& y(l)=y_{0} \cos \sqrt{k} l+\frac{y_{0}^{\prime}}{\sqrt{k}} \sin \sqrt{k} l \\
& y^{\prime}(l)=y_{0} \sqrt{k} \sin \sqrt{k} l+y_{0}^{\prime} \cos \sqrt{k} l
\end{aligned}
$$

This linear motion for a quadrupole can also be written in a matrix form:

$$
M_{\text {quadrupole }}=\left[\begin{array}{cccc}
\cosh \sqrt{k} l & \frac{1}{\sqrt{k}} \sinh \sqrt{k} l & 0 & 0 \\
-\sqrt{k} \sinh \sqrt{k} l & \cosh \sqrt{k} l & 0 & 0 \\
0 & 0 & \cos \sqrt{k} l & \frac{1}{\sqrt{k}} \sin \sqrt{k} l \\
0 & 0 & \sqrt{k} \sin \sqrt{k} l & \cos \sqrt{k} l
\end{array}\right] .
$$

This implies that when the horizontal motion is focused the vertical motion is defocused. The opposite is given when $k<0$ which results in the transport matrix

$$
M_{\text {quadrupole }}=\left[\begin{array}{cccc}
\cos \sqrt{|k|} l & \frac{1}{\sqrt{|k|}} \sin \sqrt{|k|} l & 0 & 0 \\
-\sqrt{|k|} \sin \sqrt{|k|} l & \cos \sqrt{|k|} l & 0 & 0 \\
0 & 0 & \cosh \sqrt{|k|} l & \frac{1}{\sqrt{|k|}} \sinh \sqrt{|k|} l \\
0 & 0 & \sqrt{|k|} \sinh \sqrt{|k|} l & \cosh \sqrt{|k|} l
\end{array}\right] .
$$

For short quadrupoles a good approximation can be obtained by making $l \rightarrow 0$ with $k l$ staying finite. Then the transfer matrix becomes

$$
M=\left[\begin{array}{cccc}
1 & 0 & 0 & 0 \\
-k l & 1 & 0 & 0 \\
0 & 0 & 1 & 0 \\
0 & 0 & k l & 1
\end{array}\right]
$$

where $k l=\frac{1}{f}$ and $f$ is the focal length of the lens.

For a sector dipole of bend angle $\theta$ and bend radius $\rho$ with no edge or body focusing, the Hill's equations can be written as

$$
\begin{aligned}
x^{\prime \prime}(s)+\frac{1}{\rho^{2}} x(s) & =0 \\
y^{\prime \prime}(s) & =0
\end{aligned}
$$


for which the solutions are

$$
\begin{aligned}
x(s) & =x_{0} \cos \theta+x_{0}^{\prime} \rho \sin \theta \\
x^{\prime}(s) & =-\frac{x_{0}}{\rho} \sin \theta+x_{0}^{\prime} \cos \theta \\
y(s) & =y_{0}+\rho \theta y_{0}^{\prime} \\
y^{\prime}(s) & =y_{0}^{\prime}
\end{aligned}
$$

and the linearized matrix form is

$$
M=\left[\begin{array}{cccc}
\cos \theta & \rho \sin \theta & 0 & 0 \\
-\rho \sin \theta & \cos \theta & 0 & 0 \\
0 & 0 & 1 & \rho \theta \\
0 & 0 & 0 & 1
\end{array}\right]
$$

Note that this looks like a scaled rotation in the horizontal plane, and a drift of length $l=\rho \theta$ in the vertical plane.

\subsection{DISPERSION}

We now consider particles that may have non-zero fractional momentum offset $\delta$, and consider only the coordinates $\left(x, x^{\prime}, \delta\right)$. A particle position now has a dependence on the momentum offset which is known as dispersion. The dispersion function then can be written as

The solutions are

$$
\left[\begin{array}{c}
\eta \\
\eta^{\prime} \\
1
\end{array}\right]=\left[\begin{array}{ccc}
C(s) & S(s) & D(s) \\
C^{\prime}(s) & S^{\prime}(s) & D^{\prime}(s) \\
0 & 0 & 1
\end{array}\right]\left[\begin{array}{c}
\eta \\
\eta^{\prime} \\
1
\end{array}\right]
$$

$$
\begin{aligned}
\eta(s) & =\frac{\left[1-S^{\prime}(s)\right] D(s)+S(s) D^{\prime}(s)}{2(1-\cos \mu)} \\
\eta^{\prime}(s) & =\frac{[1-C(s)] D^{\prime}(s)+C^{\prime}(s) D(s)}{2(1-\cos \mu)} .
\end{aligned}
$$

For a sector dipole the full $6 \mathrm{D}$ matrix which connects the momentum spread to its transverse coordinates is then be represented by

$$
M=\left[\begin{array}{cccccc}
\cos \theta & \rho \sin \theta & 0 & 0 & 0 & \rho(1-\cos \theta) \\
(-1 / \rho) \sin \theta & \cos \theta & 0 & 0 & 0 & \sin \theta \\
0 & 0 & 1 & \rho \theta & 0 & 0 \\
0 & 0 & 0 & 1 & 0 & 0 \\
-\sin \theta & -\rho(1-\cos \theta) & 0 & 0 & 1 & -\rho(\theta-\sin \theta) \\
0 & 0 & 0 & 0 & 0 & 1
\end{array}\right]
$$


where $\rho$ is the bend radius and $\theta$ is the bending angle.

\subsubsection{FIRST ORDER ACHROMAT}

A beam bending through a dipole develops a dispersion that adds up or cancels out depending on the lattice. A lattice that cancels out the dispersion to first order is known as a first order achromat.

\subsection{BUNCH COMPRESSION}

A bunch compressor works as a device that shortens the longitudinal bunch length. This is done using the momentum spread of the bunch. The particles has a slight momentum deviation from the design particle defined by $\delta \equiv\left(p-p_{0}\right) / p_{0}$. With this when a beam travels in curved trajectory, the particle that has different momentum offset travels in slightly different path given by $L=\oint d s$. The fractional deviation of this path length divided by the fractional deviation of the momentum is called the momentum compaction[11] denoted by

$$
\alpha_{p}=\frac{d L}{L} / \frac{d p}{p}=\frac{p}{L} \frac{d L}{d p}
$$

Taking the path length for an off momentum particle as $L+\Delta L=\oint d \sigma$ and the fact that both design particle and off momentum one has the same infinitesimal azimuthal angle

$$
\frac{d \sigma}{\rho+x_{p}}=\frac{d s}{\rho} \longrightarrow d \sigma \equiv\left(1+\frac{x_{p}}{\rho}\right) d s .
$$

With this we have $d \sigma=\left(1+x_{p} / \rho\right) d s$ that can be used to find the fractional path length difference $\Delta L=\oint x_{p} / \rho d s$. We then can rewrite the momentum compaction in terms of the dispersion as

$$
\alpha_{p}=\frac{1}{L} \oint \frac{\eta(s)}{\rho(s)} d s .
$$

\subsubsection{RF CHIRP AND R56}

High peak currents are required in light sources, linear colliders and for advanced accelerator physics studies. One of the ways to get a shorter bunch is to use a bunch compressor at the end of the machine. An electron bunch accelerated through an RF system at a phase $\left(\phi_{R F}\right)$ producing a chirp, which is a correlation between energy and longitudinal position. 
Depending on the energy above or below transition the chirp can be either positive or negative. A positively chirped beam has its lowest energy particles at the tail of the bunch [13] as seen in the left figure of Fig. 10
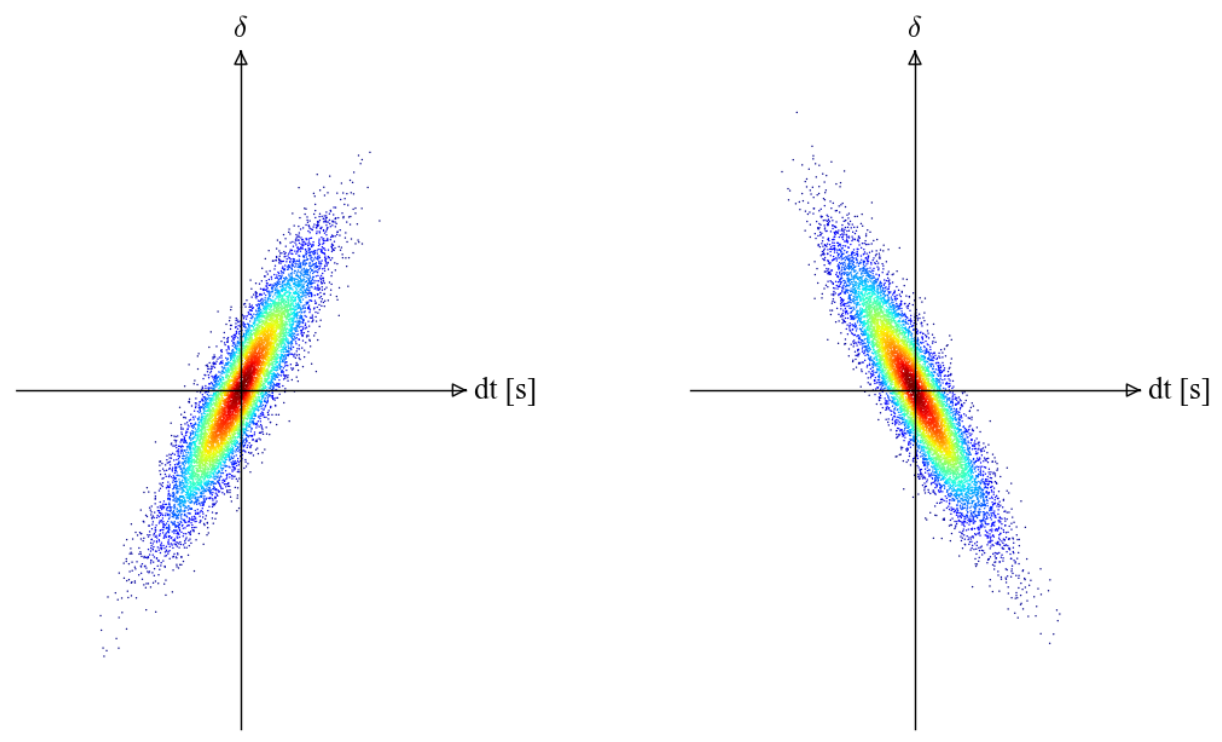

FIG. 10: RFchirp

A bunch compressor deals with both transverse (to keep the bunch focused throughout the system) and longitudinal planes (compressing). An ideal example of a bunch going in to and coming out of a compressor can be shown in the figure below 

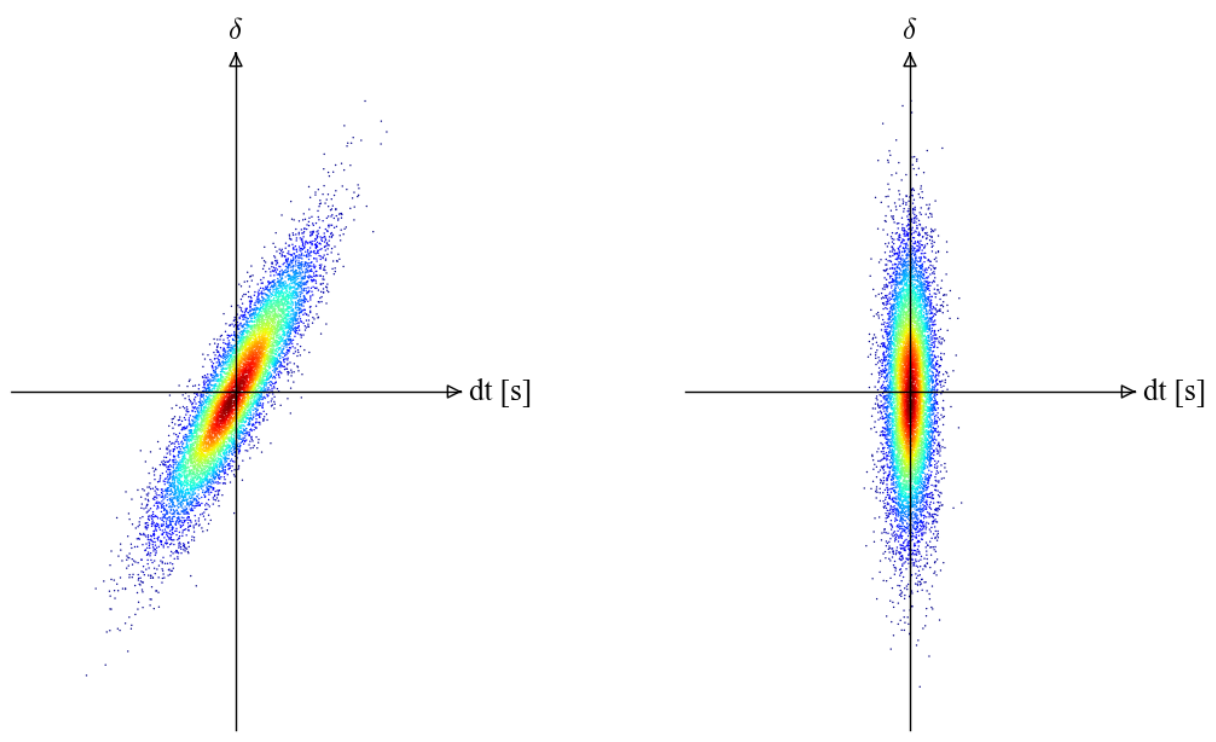

FIG. 11: A bunch profile before and after going through a bunch compressor.

When a beam goes through a dipole, the different energy particles get bent in slightly different angles. This is equivalent to a white light ray passing through a prism. By using this concept it can be arranged so that the high energy particle, that arrives relatively earlier than the synchronous particle and the lower energy particle that arrives relatively after the synchronous, comes almost at the same time on the target. The important thing to remember is that the whole compressor should be designed in a way that outputs an achromatic beam when the input is also achromatic.

\subsubsection{DOUBLE BEND ACHROMAT}

The simplest lattice that keeps the achromaticity (to the first order) of the beam intact is a double bend achromat(DBA). First introduced by Panofsky [14] a DBA is designed with two dipoles and one quadrupole in the middle. When an achromatic beam enters the dipole, dispersion is introduced, as it exits the dipole and drift in free space the dispersion increases. The middle quadrupole is to introduce a focusing effect that scales with the displacement from the center of the beamline. By using the symmetry of the lattice, beam achromaticity will be preserved at the end of the second dipole as seen in Fig. 12. 


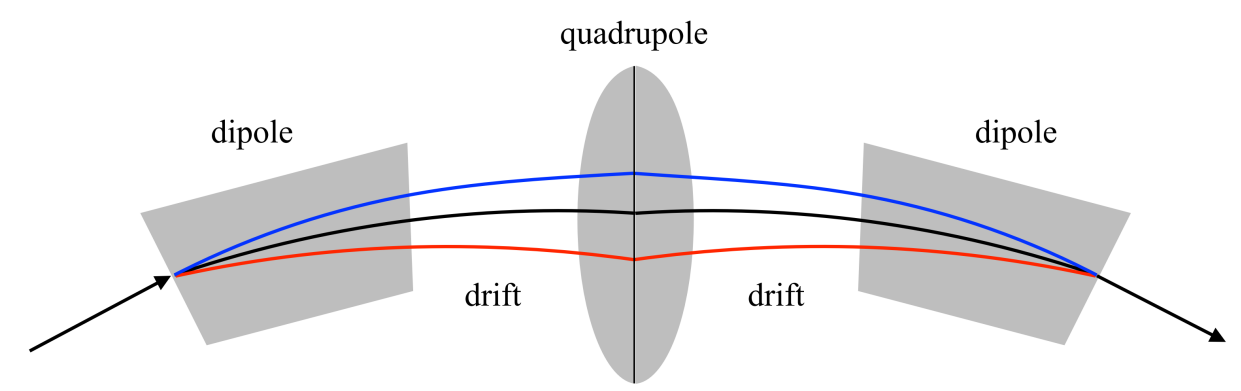

FIG. 12: Double bend achromat

\subsubsection{FOUR BEND ACHROMAT}

The dependence of path length $s$ on fractional momentum offset $\delta$ can be expanded in a power series as

$$
s(\delta)=R_{56} \delta+T_{566} \delta^{2}+U_{5666} \delta^{3}+\cdots
$$

where $R_{56}$ is the linear compression of the system, and $T_{566}$ and $U_{5666}$ are the higher order nonlinear compression terms. A basic example for a bunch compressor is a chicane consisting of four dipoles and no quadrupoles.

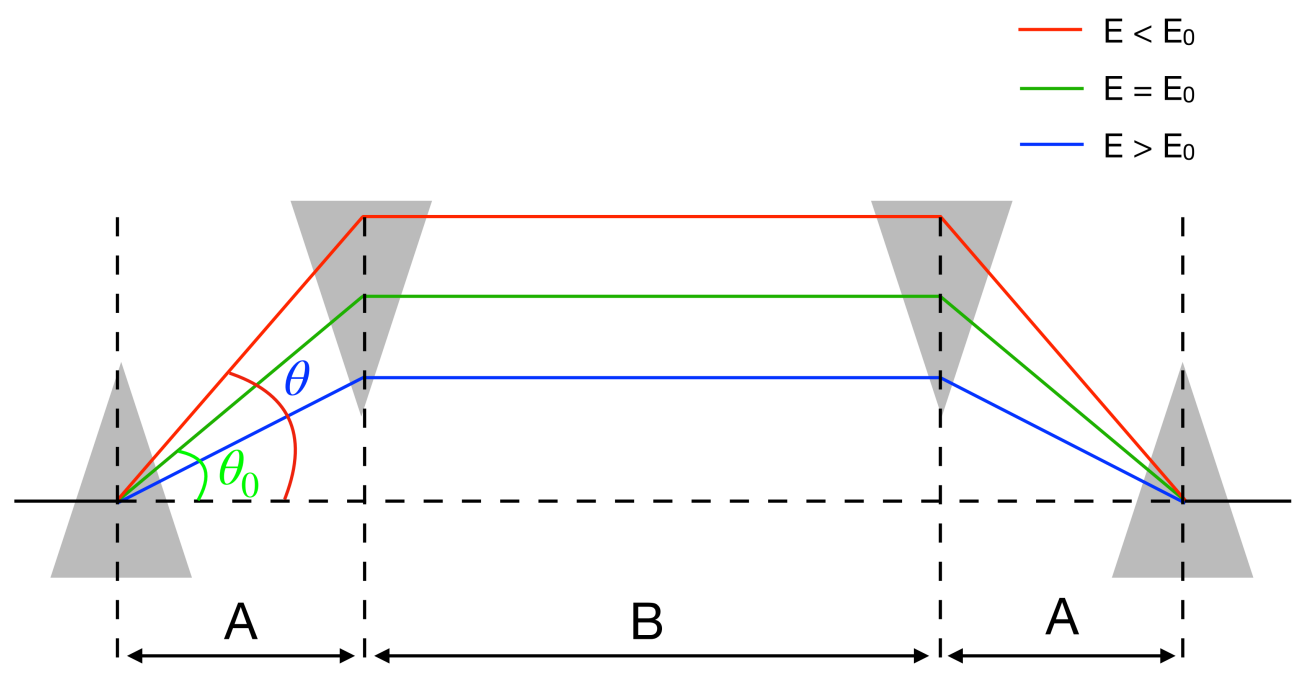

FIG. 13: 4 dipole chicane 
The path length for a particle traveling in the design orbit from start to end in this chicane is given by

$$
s=\frac{2 A}{\cos (\theta)}+B \simeq \frac{2 A}{1-\theta^{2}}+B \simeq 2 A+2 A \theta^{2}+B .
$$

If the the particle has a small energy deviation $\delta$ the bending angle will be changed to

$$
\theta=\frac{\theta_{0}}{1+\delta}
$$

Then we can rewrite Eq. 30 as

$$
s=2 A+2 A\left(\frac{\theta_{0}}{1+\delta}\right)^{2}+B .
$$

Then the path length in the chicane is given by

$$
\Delta s=s(\delta)-s(0) \simeq A\left(\frac{\theta_{0}}{1+\delta}\right)^{2}-A \theta_{0}^{2}=\frac{1}{2} R_{56}\left(1-\frac{1}{(1+\delta)^{2}}\right) .
$$

Using a Taylor expansion around $\delta=0$ we can write

$$
\Delta s \simeq R_{56} \delta-\frac{3}{2} R_{56} \delta^{2}+2 R_{56} \delta^{3}-\cdots
$$

where the higher order terms are related to the first order momentum compaction by

$$
\begin{aligned}
T_{566} & \simeq \frac{3}{2} R_{56} \\
U_{5666} & \simeq 2 R_{56} .
\end{aligned}
$$

Four dipole bunch compressors have been traditionally used to obtain shorter bunch lengths.

\subsection{LONGITUDINAL SYNCHROTRON DYNAMICS}

Longitudinal motion is treated as nonlinear due to the restoring force being sinusoidal. Particles that has higher momentum are harder to bend. So their trajectory circle is slightly higher than the synchronous one. For particles with slightly less energy the trajectory circle is smaller. Above transition this results in higher energy particles arriving late and lower energy particles arriving early. 


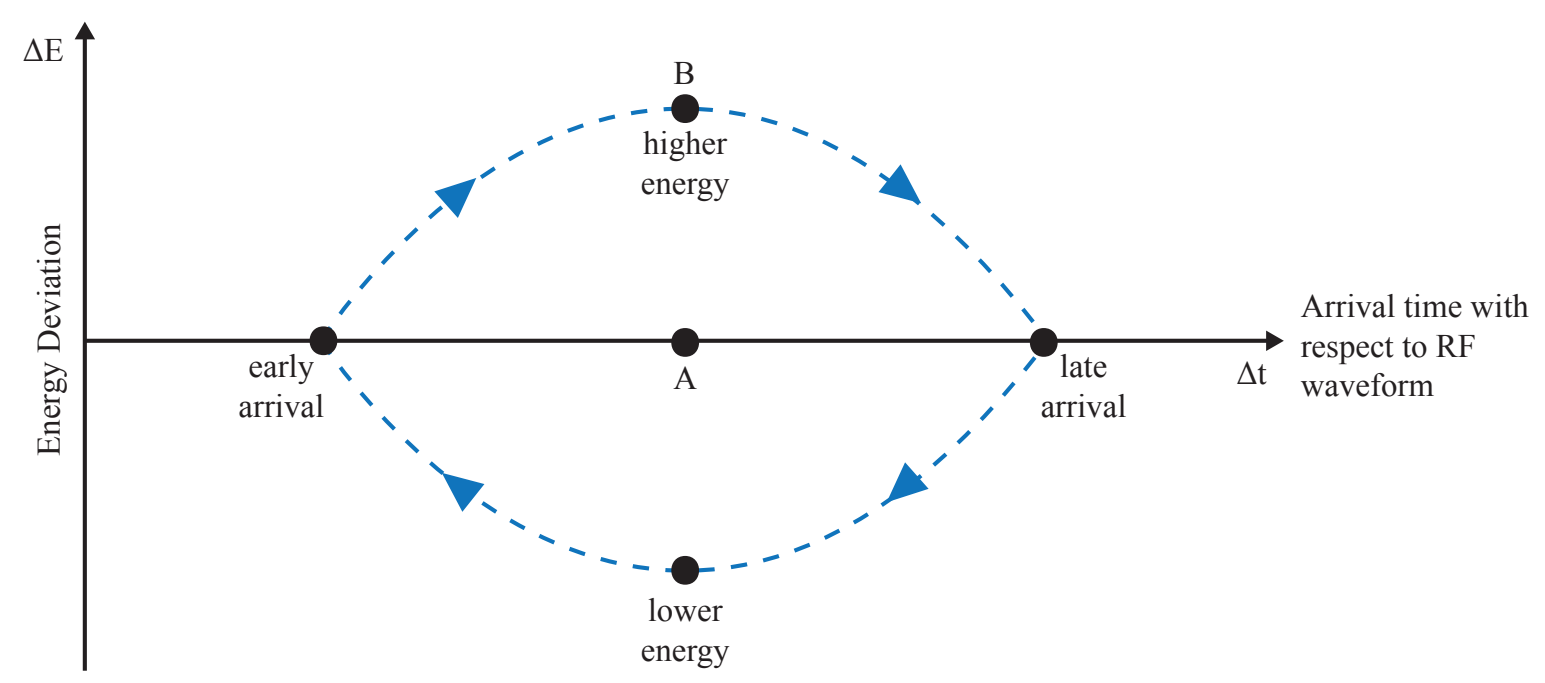

FIG. 14: Energy vs time

\subsubsection{BASIC LONGITUDINAL SYNCHROTRON MOTION}

The angular revolution frequency of a synchrotron is specified by

$$
\omega_{s}=\frac{2 \pi \beta c}{L}
$$

where $L$ is the accelerator circumference, and $c$ is the speed of light. The second part of the Lorentz force describes the electric field that is use to control the overall momentum and thus the longitudinal motion of the beam, and provide longitudinal focusing:

$$
\frac{d \vec{p}}{d t}=q \vec{E}
$$

For longitudinal focusing, we apply a longitudinal electric field to the synchronous particle every turn of the form

$$
V=V_{0} \sin \phi_{s}
$$

which, for a particle with charge $q$, gives an energy gain of $\Delta U_{s}=q V \sin \phi_{s}$ per turn.

In practice we apply a sinusoidal periodic longitudinal electric field through RF cavities. These RF cavities have a frequency $\omega_{\text {rf }}$ such that each synchronous particle in successive bunches experiences the same voltage given by Eq. 38. Then the electric field can be written as

$$
\vec{E}(s, t)=\hat{s} V \sin \left(\omega_{\mathrm{rf}} t+\phi_{\mathrm{s}}\right) \sum_{n=-\infty}^{\infty} \delta(s-n L)
$$


where the angular RF frequency $\omega_{\text {rf }}$ is an integer multiple of the angular revolution frequency

$$
\omega_{\mathrm{rf}}=h \omega_{\mathrm{s}}
$$

The integer multiple denoted by $h$ is called the harmonic number. This $h$ defines the number of "buckets", or places where bunches would be longitudinally focused, in the synchrotron.

\subsubsection{LONGITUDINAL EMITTANCE}

Just as transverse emittances define the extent of the beam distribution in transverse phase space, the longitudinal emittance defines the extent of the beam in longitudinal phase space. This is complicated by the fact that the reference particle may not have zero longitudinal coordinates if the synchronous phase $\phi_{s}$ is non-zero.

The rms emittance in the longitudinal plane is calculated in ESME using its longitudinal coordinates $(\theta, E)$ for a distribution of $N$ macroparticles by

$$
\epsilon=\frac{T_{\mathrm{rev}}}{2 N} \sqrt{\sum\left(\theta_{i}-\bar{\theta}\right)^{2} \sum\left(E_{i}-\bar{E}\right)^{2}-\left[\sum\left(\theta_{i}-\bar{\theta}\right)\left(E_{i}-\bar{E}\right)\right]^{2}}[\mathrm{eV}-\mathrm{s}]
$$

where the sums are over the particle distribution. Multiplying this by six gives an emittance value containing $95 \%$ of the particles. If the energy spread of the bunch is known then the 95\% emittance can also be calculated by [15]

$$
S_{95 \%}=\frac{3}{h f_{0}} \sqrt{\frac{-2 \pi h \eta E_{s}^{3}}{\beta^{2} e V \cos \phi_{s}}}\left(\frac{\sigma_{E}}{E_{s}}\right)^{2} .
$$

If both the rms bunch length and the energy spread are known, then the emittance can be found with

$$
S_{95 \%}=6 \pi \sigma_{t} \sigma_{d E}
$$

\subsubsection{EQUATIONS OF MOTION}

To describe the motion of the particles other than the synchronous particle we define

$$
\begin{aligned}
U & =U_{s}+\delta U \\
p & =p_{s}+\delta p \\
\omega & =\omega_{s}+\delta \omega \\
\tau & =\tau_{s}+\delta \tau \\
\phi & =\phi_{s}+\delta \phi_{s}
\end{aligned}
$$


For the design particle arriving at the cavity the phase of the rf voltage is taken as $\phi_{s}$ and the for the test particle it's $\phi$. Then the phase difference between the design and the test particle is given by

$$
\varphi=\phi-\phi_{s}
$$

The energy gain a particle gets per turn passing through a cavity is

$$
\Delta U=q V \sin \phi
$$

The change in energy between the test and the design particle after one turn is then given by

$$
\delta(\delta U)=\delta U-\delta U_{s}=q V\left(\sin \phi-\sin \phi_{s}\right) .
$$

At a slowly varying oscillation about the synchronous energy we can rewrite the Eq. 47

$$
\frac{d(\delta U)}{d t} \simeq \frac{\Delta(\delta U)}{\tau_{s}}=\frac{q V}{2 \pi} \omega_{s}\left(\sin \phi-\sin \phi_{s}\right)
$$

with defining the variable $W=\delta U / \omega_{r f}=-\left(U-U_{s}\right) / \omega_{r f}$ Eq. 48 becomes

$$
\frac{d W}{d t}=\frac{q V}{2 \pi h}\left(\sin \phi_{s}-\sin \phi\right) .
$$

The change in phase difference is

$$
\Delta \varphi \simeq \frac{d \varphi}{d t} \tau_{s}=\omega_{r f} \delta t
$$

where $\delta t$ is the time delay between the test and design particle arriving at a cavity. After one revolution the change in $\delta t$

$$
\Delta(\delta t)=\tau-\tau_{s}=\delta \tau=-\eta_{t r} \tau \frac{d p}{p} .
$$

Using the relation $\frac{\delta p}{p}=\left(1 / \beta^{2} U\right) \delta U$ an equation for $\dot{\varphi}$ can be written as

$$
\frac{d \varphi}{d t}=\frac{\omega_{r f}^{2} \eta_{t} r}{\beta^{2} U_{s}} W
$$

Plots of constant energy for these equations of motion are shown in Fig. 15. 


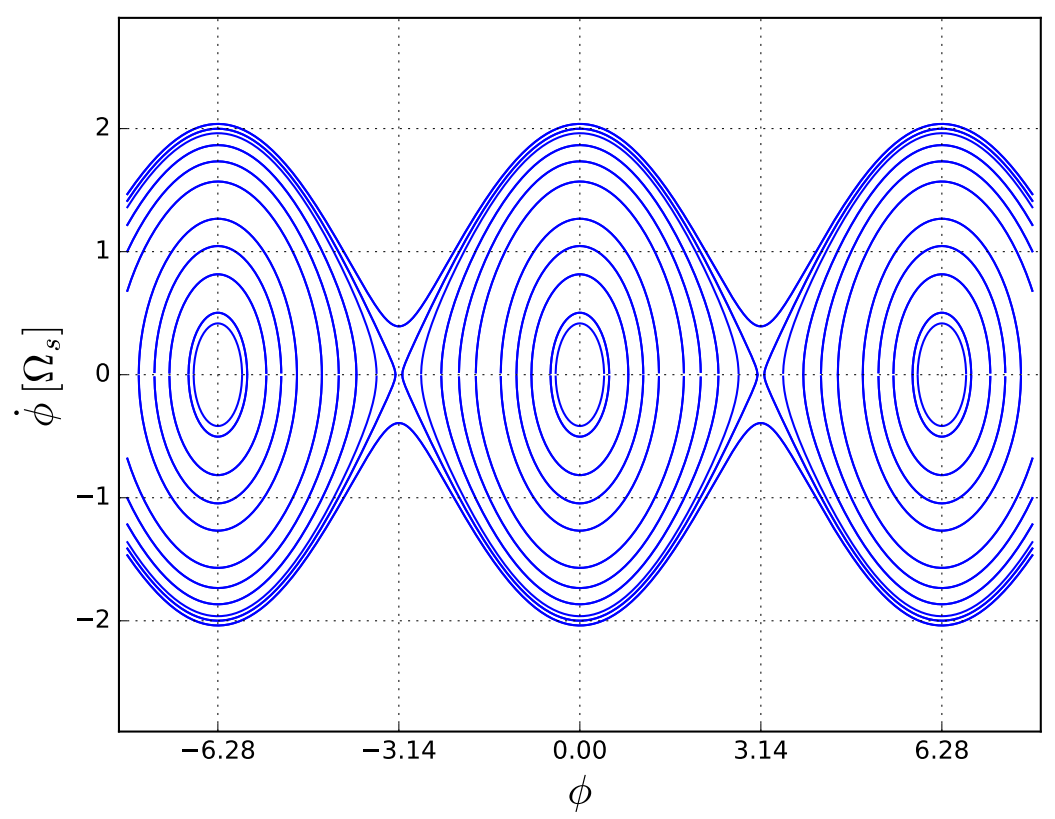

FIG. 15: Phase space plots for $\phi_{s}=0$.

\subsubsection{HAMILTONIAN AND CANONICAL COORDINATES}

For small synchrotron tunes, the average longitudinal Hamiltonian can be written as

$$
H(\varphi, W)=-p_{s}+\left(\frac{h^{2} \omega_{s}^{2} \eta_{\mathrm{tr}}}{2 \beta^{3} U_{s} c}\right) W^{2}-\frac{q V}{2 \pi \beta c h}\left[\cos \left(\phi_{s}+\varphi\right)+\varphi \sin \phi_{s}\right]
$$

where $s$ is the time coordinate, $(\varphi, W)$ are the canonical coordinates (with $\varphi \equiv \phi-\phi_{s}$ and $W \equiv\left(U_{s}-U\right) / \omega_{\mathrm{rf}}, \varphi$ is the RF phase distance to the synchronous particle, $W$ is the normalized total energy difference from the synchronous particle at phase $\phi_{s}$ ), and $L$ is the synchrotron circumference. The angular RF frequency $\omega_{\mathrm{rf}}=h \omega_{\mathrm{s}}$ where $\omega_{\mathrm{s}}$ is the angular synchronous particle revolution frequency.

\subsubsection{SYNCHROTRON FREQUENCY/TUNE}

From the Hamiltonian, the equations of motion (where ${ }^{\prime} \equiv d / d s$ ) are

$$
\begin{aligned}
\varphi^{\prime} & =\frac{\partial H}{\partial W}=\frac{\omega_{\mathrm{rf}}^{2} \eta_{\mathrm{tr}}}{\beta^{3} U_{s} c} W \\
W^{\prime} & =-\frac{\partial H}{\partial \varphi}=\frac{q V}{L \omega_{\mathrm{rf}}}\left[\sin \phi_{s}-\sin \left(\phi_{s}+\varphi\right)\right]
\end{aligned}
$$

or, since ${ }^{\prime} \equiv d / d s=(d / d t)(d s / d t)=(\beta c)(d / d t), L \omega_{\mathrm{rf}}=2 \pi(\beta c) h$, and $\sin \left(\phi_{s}+\varphi\right)=$ 
$\sin \phi_{s} \cos \varphi+\cos \phi_{s} \sin \varphi$

$$
\begin{aligned}
\ddot{\varphi} & =(\beta c)^{2} \varphi^{\prime \prime}=(\beta c)^{2}\left[\frac{\omega_{\mathrm{rf}}^{2} \eta_{\mathrm{tr}}}{\beta^{3} U_{s} c}\right]\left[\frac{q V}{L \omega_{\mathrm{rf}}}\left[\sin \phi_{s}-\sin \left(\phi_{s}+\varphi\right)\right]\right] \\
& =\Omega_{s}^{2}\left[\sin \varphi+(1-\cos \varphi) \tan \phi_{s}\right]
\end{aligned}
$$

where

$$
\Omega_{s} \equiv \sqrt{\frac{q V}{U_{s}} \frac{h \eta_{\mathrm{tr}} \omega_{s}^{2}}{2 \pi \beta^{2}} \cos \phi_{s}} \quad[\mathrm{rad} / \mathrm{s}]
$$

is the synchrotron angular frequency and

$$
Q_{s} \equiv \frac{\Omega_{s}}{\omega_{s}}=\sqrt{\frac{q V}{U_{s}} \frac{h \eta_{\mathrm{tr}}}{2 \pi \beta^{2}} \cos \phi_{s}} \quad[1 / \text { turn }]
$$

is the synchrotron tune. Unlike the betatron oscillations where the particle goes through several oscillations per turn, the synchrotron oscillations take several (thousands or more) turns to complete one oscillation. Using the relation $f=1 / T$ we can find the synchrotron period; this synchrotron period is plotted for different RF amplitudes for the JLEIC ion collider ring at $100 \mathrm{GeV}$ in Fig. 16.

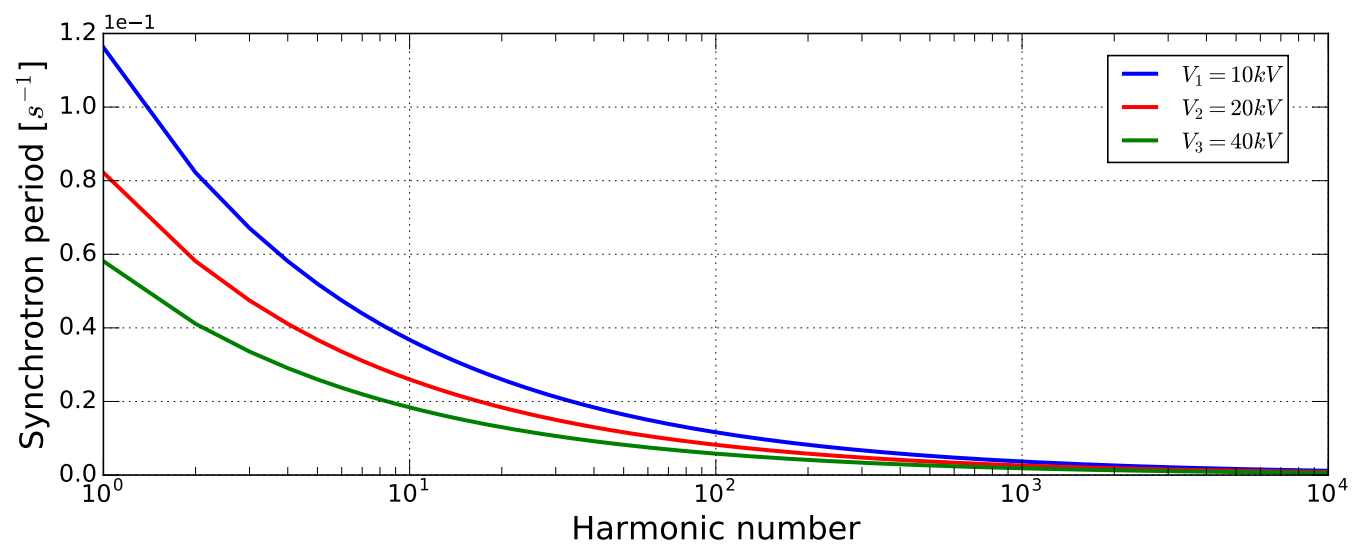

FIG. 16: Synchrotron period vs the harmonic number for different RF amplitudes.

\subsubsection{BUCKET HEIGHT AND AREA}

The bucket height $\hat{W}$ and area $\mathcal{A}$ are easiest to calculate for the stationary case $\phi_{s}=0$. Then we can set the Hamiltonian at the unstable fixed point $H(\varphi=\pi, W=0)$ equal to the Hamiltonian at the stable fixed point $H(\varphi=0, W=\hat{W})$ which gives an expression to the bucket height 


$$
\frac{\hat{U}}{U_{s}}=\sqrt{\frac{2 q V}{U_{s}} \frac{\beta^{2}}{\pi h \eta_{\mathrm{tr}}}} \approx \frac{d E}{E} \approx \frac{d p}{p} \quad \text { bucket height [unitless] }
$$

which is consistent with other sources. Note the scaling with $V$ and $h$. To keep the same bucket height for changing RF parameters at the same energy, $V / h$ must stay constant.

The bucket area $\mathcal{A}$ can be calculated by setting the Hamiltonian to its value at the unstable fixed point, $H(\varphi=\pi, W=0)$, integrating it from $\varphi: 0 \rightarrow \pi$, and multiplying by four. It is also best to convert back to $U$ units so this integral is really integrating energy:

$$
\begin{gathered}
H(\varphi, W)=-p_{s}+\frac{h^{2} \omega_{s}^{2} \eta_{\mathrm{tr}}}{2 \beta^{3} U_{s} c} W^{2}-\frac{q V}{2 \pi \beta c h} \cos \varphi \\
=H(\varphi=\pi, W=0)=-p_{s}+\frac{q V}{2 \pi \beta c h} \\
\Rightarrow W=\sqrt{\frac{\beta^{2} U_{s}}{h^{3} \omega_{s}^{2} \eta_{\mathrm{tr}}} \frac{q V}{\pi}(1+\cos \varphi)} \\
\Rightarrow U=\sqrt{\frac{\beta^{2} U_{s}}{h \eta_{\mathrm{tr}}} \frac{q V}{\pi}(1+\cos \varphi)} \\
\mathcal{A}=4 \sqrt{\frac{\beta^{2} U_{s}}{h \eta_{\mathrm{tr}}} \frac{q V}{\pi}} \int_{0}^{\pi} \sqrt{(1+\cos \varphi)} d \varphi
\end{gathered}
$$

using the integral value $\int_{0}^{\pi} \sqrt{(1+\cos \varphi)} d \varphi=2 \sqrt{2}$,

$$
\mathcal{A}=8 \sqrt{\frac{2 \beta^{2} U_{s}}{h \eta_{\mathrm{tr}}} \frac{q V}{\pi}} .
$$

This area is in units of eV-rad (in the RF wavelength). The usual convention is to use units of $\mathrm{eV}$-s for bucket areas and longitudinal emittances, so we should convert radians in the RF wavelength to time. The time it takes the beam to go around the circumference of the machine $L$ is $L /(c \beta)$; in this time the $\mathrm{RF}$ goes through $h$ oscillations or $2 \pi h$ radians. So the appropriate conversion to time units is to multiply by

$$
\frac{L}{(c \beta)(2 \pi h)}=\frac{2 \pi(\beta c) h / \omega_{\mathrm{rf}}}{(c \beta)(2 \pi h)}=\frac{1}{\omega_{\mathrm{rf}}} \frac{1}{h \omega_{s}} .
$$

This gives an equation for the bucket area

$$
\mathcal{A}=\frac{8 \beta}{h \omega_{s}} \sqrt{\frac{2 U_{s}}{h \eta_{\mathrm{tr}}} \frac{q V}{\pi}}=\frac{8 \beta}{\pi f_{\mathrm{rf}}} \sqrt{\frac{U_{s}}{2 h \eta_{\mathrm{tr}}} \frac{q V}{\pi}}=\frac{8 \hat{U}}{\omega_{\mathrm{rf}}} \quad[\mathrm{eV}-\mathrm{s}] .
$$

If one keeps the same bucket height $\hat{U}$ during bunch splitting by keeping $V / h$ constant, the area of each bucket also proportionally goes down by the change in RF frequency. 
However, the synchrotron tune $Q_{s}$ is proportional to $\sqrt{h V}$, so doubling both $h$ and $V$ for each bunch splitting means that $Q_{s}$ goes up by a factor of 2 for each bunch splitting, or $2^{n}$ for $n$ bunch splittings.
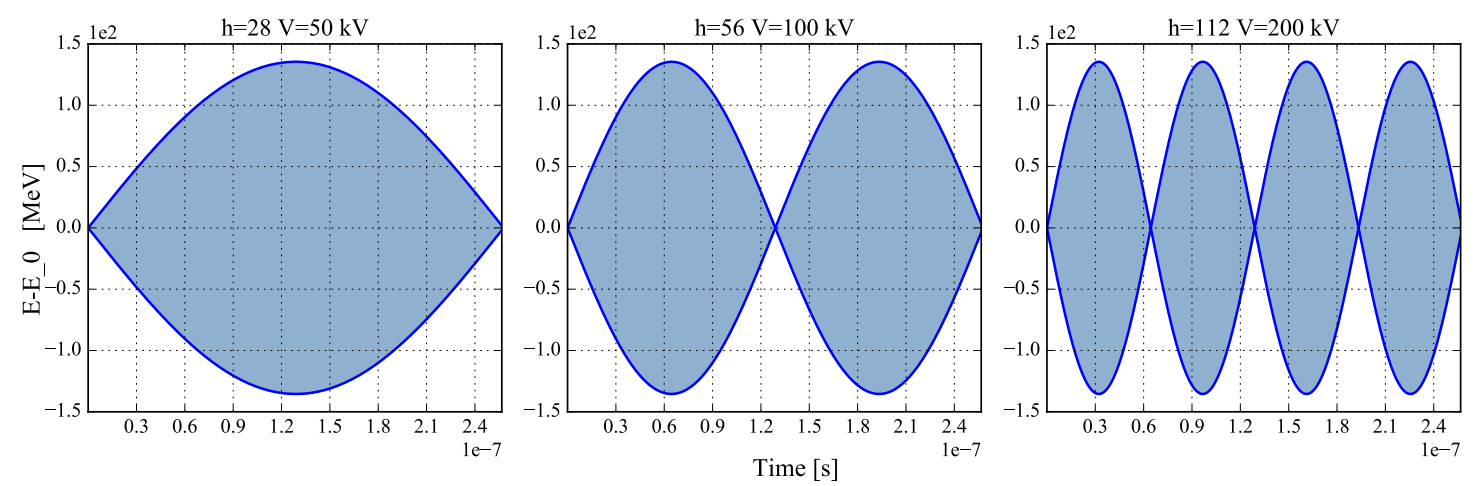

FIG. 17: RF bucket change with change in the harmonic number and RF amplitude while the $h: V$ ratio kept constant. The area of each bucket are $44.46 \mathrm{eV}-\mathrm{s}, 22.23 \mathrm{eV}-\mathrm{s}$ and 11.11 eV-s respectively.

For a beam with energy above transition a comparison between the RF waveform and a bunch inside the bucket is shown in Fig. 18. The bunch is centered at $\phi=180^{\circ}$

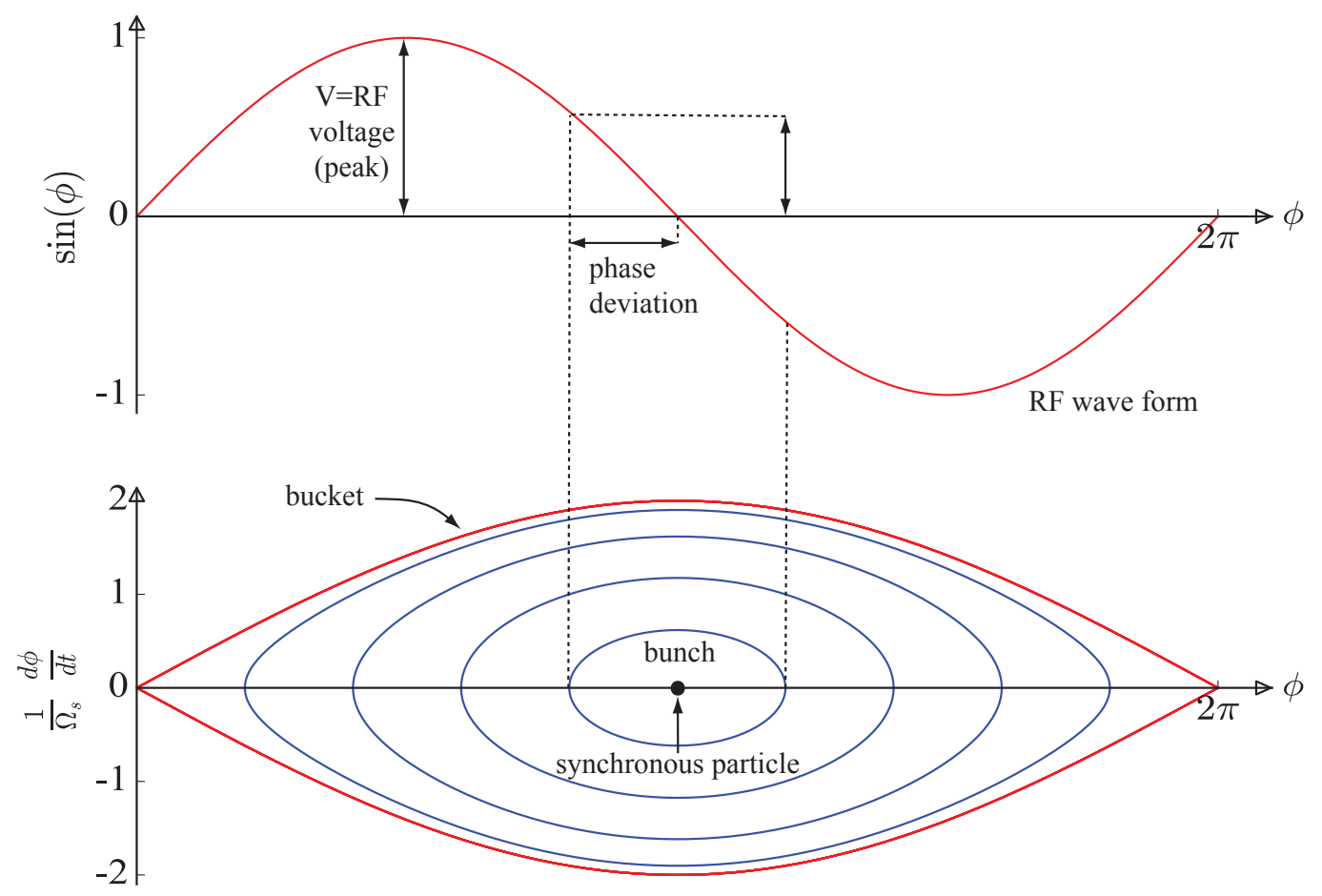

FIG. 18: RF wave comparison to the bucket and the bunch inside it. 


\subsection{ADIABATICITY PARAMETER}

RF ramps that are being changed needs to be ramped adiabatically to keep the phase space dilution to a minimum. In an adiabatic process the particle distribution is continuously at equilibrium with the instantaneous values of the system. To measure the degree of such a process we use the term called the adiabaticity parameter [16] given by

$$
\epsilon_{a}=\frac{1}{\omega_{s}^{2}}\left|\frac{d \omega_{s}}{d t}\right|=\frac{1}{2 \pi}\left|\frac{d T_{\mathrm{s}}}{d t}\right| .
$$

For a process to be adiabatic it should generally be $\epsilon<<1$.

\subsubsection{ISO-ADIABATIC VOLTAGE RAMPS}

As the RF voltage amplitude changes, so does the synchrotron tune. So the ramping rate of the RF voltage can be changed as the time goes on. This is represented by iso-adiabatic curves (Fig. 19) and was considered in the GA optimization to see if it can be used to further reduce the split time while keeping the emittance growth low. The synchrotron period $\left(T_{\mathrm{s}}\right)$ is related to the $\mathrm{RF}$ voltage amplitude as $T_{\mathrm{s}}=2 \pi a V_{\mathrm{rf}}^{-\frac{1}{2}}$. Substituting in the adiabaticity parameter a relation can be obtained as $\epsilon_{a} d t=(1 / 2) a V^{-3 / 2} d V$. Integrating gives a relation for the adiabaticity parameter in terms of ramp time and the RF amplitudes as

$$
\epsilon_{a}=a \frac{1}{t_{\mathrm{ramp}}}\left(\frac{1}{\sqrt{V_{i}}}-\frac{1}{\sqrt{V_{f}}}\right) .
$$

An indefinite integration gives the form $\epsilon t+c=a V^{-1 / 2}$ which using the Eq. 68 can be reformed to

$$
V(t)=\frac{1}{\left(\frac{t}{t_{\mathrm{ramp}}}\left(\frac{1}{\sqrt{V_{i}}}-\frac{1}{\sqrt{V_{f}}}\right)+c\right)^{2}} .
$$

With the boundary conditions $V(0)=V_{i}$ and $V\left(t_{\text {ramp }}\right)=V_{f}$ this can be written as

$$
V(t)=\frac{V_{i}}{\left(1-\frac{t}{t_{\mathrm{ramp}}}\left(1-\frac{\sqrt{V_{i}}}{\sqrt{V_{f}}}\right)\right)^{2}} .
$$




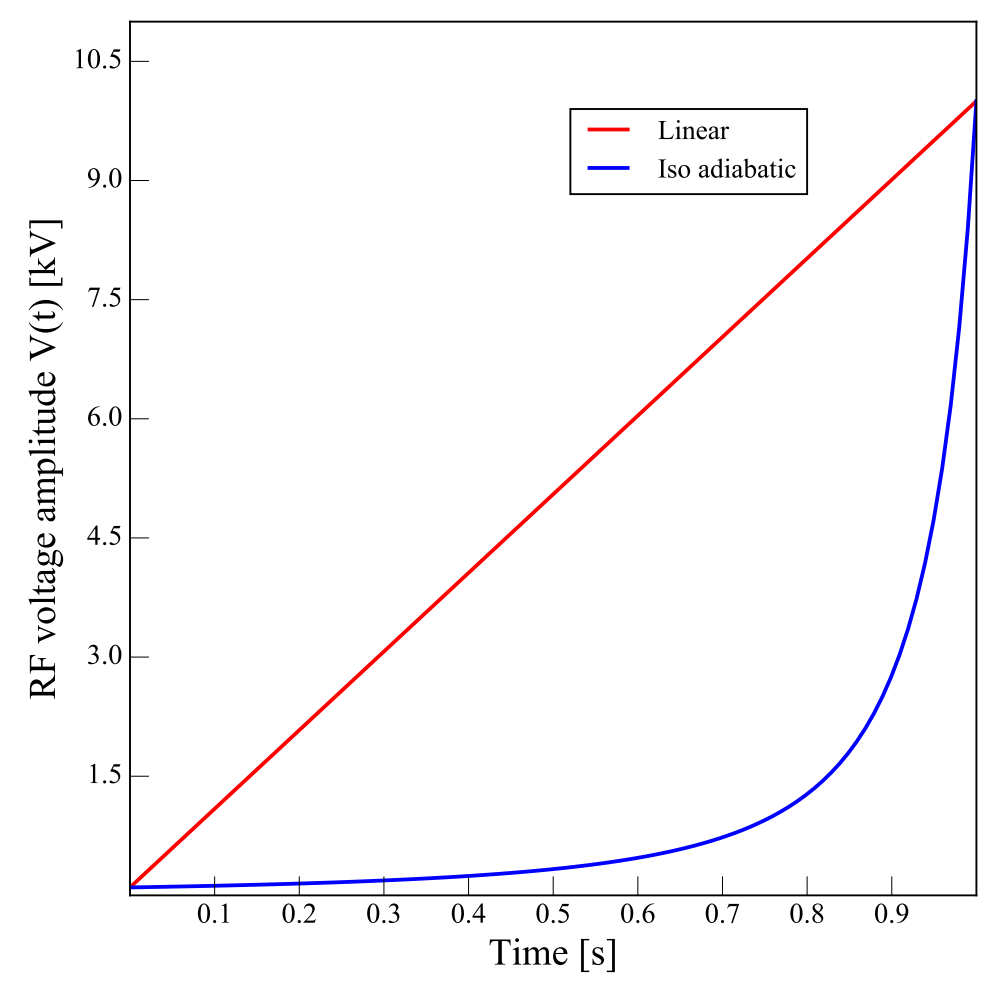

FIG. 19: An iso-adiabatic voltage ramp compared to a linear ramp. With $V_{i}=100 \mathrm{~V}$, $V_{f}=10 \mathrm{kV}$ and $T_{\text {ramp }}=1 \mathrm{~s}$.

In an iso adiabatic ramp the final voltage for debunching (or initial voltage for rebunching) is selected to be non zero. In a debunching ramp the voltage reaches its final value and then steps down to zero. 


\section{CHAPTER 4}

\section{TUNABLE BUNCH COMPRESSOR}

\subsection{INTRODUCTION}

There is significant interest in X-ray sources beyond those available at large thirdgeneration, lab-based synchrotron light sources. Medical phase contrast imaging and industrial applications have motivated development of compact light sources based on inverse Compton scattering (ICS) [17] that provide single beamline operation at a reasonable cost.

Inverse Compton scattering is an electron-photon scattering process where the photons scatter in to higher energies off of relativistic electrons. In this case the photons gains energy that is being lost by the electrons.

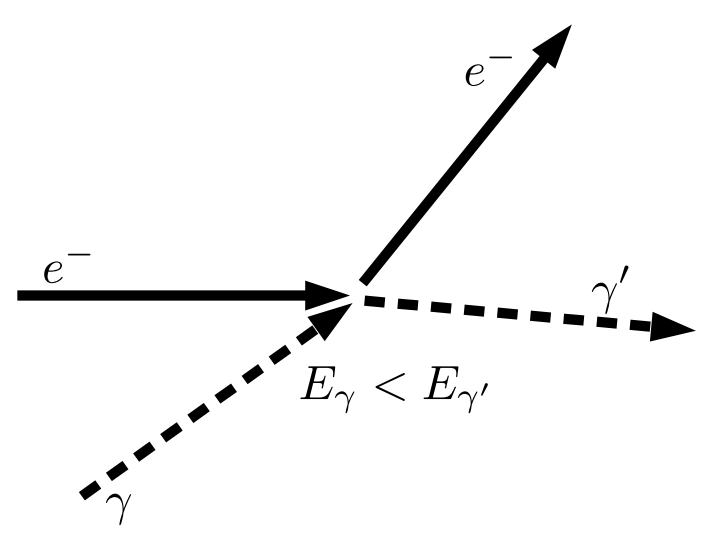

FIG. 20: Inverse Compton scattering.

A compact ICS source was designed at the ODU Center for Accelerator Science (CAS) with an X-ray energy of up to $12 \mathrm{keV}$, flux of approximately $1.6 \times 10^{14}$ photon/s, and average brilliance of $1.5 \times 10^{15}$ photon $/\left(\mathrm{s} \cdot \mathrm{mm}^{2} \cdot \mathrm{mrad}^{2} \cdot 0.1 \% \mathrm{BW}\right)$ [18] [4].

This ICS source consists of an optimized $500 \mathrm{MHz}$ superconducting electron gun, 4K $500 \mathrm{MHz}$ superconducting spoke cavity linac [19], and a bunch compressor to provide short, high-brightness electron bunches to collide with an incoming laser beam to produce inverse Compton scattered photons. The electron beam requirements are shown in Table 4 . The 
bunch length requirement of a few ps and energy spread requirement of $3 \times 10^{-4}$ indicate the need for small longitudinal emittance $(<7 \mathrm{eV} \cdot \mathrm{s})$ and well-corrected bunch compression. A practical system will also have tunable bunch compression to optimize the scattered photon beam.

TABLE 4: Electron beam parameters at collision.

\begin{tabular}{lcc}
\hline \hline Parameter & Value & Units \\
\hline Energy & 25 & $\mathrm{MeV}$ \\
Bunch charge & 10 & $\mathrm{pC}$ \\
Repetition rate & 100 & $\mathrm{MHz}$ \\
Average current & 1 & $\mathrm{~mA}$ \\
Transverse rms normalized emittance & 0.1 & $\mathrm{~mm} \cdot \mathrm{mrad}$ \\
$\alpha_{x, y}$ & 0 & - \\
$\beta_{x, y}$ & 5 & $\mathrm{~mm}$ \\
$\sigma_{x, y}$ & 3 & $\mu \mathrm{m}$ \\
FWHM bunch length & $3.0(0.9)$ & $\mathrm{psec}(\mathrm{mm})$ \\
RMS energy spread & 7.5 & $\mathrm{keV}$ \\
\hline \hline
\end{tabular}

The points that were taken under consideration for the bunch compressor design were to keep the overall space as small as possible, and to have a large and tunable momentum compaction $M_{56}$.

\subsection{DESIGN PHILOSOPHY}

Traditionally achromatic chicanes are used for magnetic bunch compression, with $M_{56}<0$ and $T_{566}>0$ [20]. However, these chicanes are not very compact and cancel dispersion only to odd orders. $M_{56}$ tunability is also directly connected to the chicane transverse dimension, and so is limited.

For a compact design with tunable $M_{56}$, 4-dipole s-chicanes were evaluated, that alternate the bending directions of selected dipole pair. A chicane of this type was also investigated for the final bunch compressor of the TTF-FEL [21]. S-shaped compressors are dispersion free to all orders, have greater $M_{56}$ tunability, and can be wrapped in on themselves with large 
central bend angles to be made compact. As described in [21], $M_{56}$ is tunable by adjusting the central dipole angle.

Initial designs considered combining quadrupoles in the compressor with quadrupoles outside the compressor to tune the final focus. However, the aggressiveness of the design final focusing to $\beta^{*}=5 \mathrm{~mm}$ is best handled with a separate independent low-beta focus section. This sacrifices some small measure of compactness of the design, but greatly improves the orthogonality of tuning knobs for achromaticity, $M_{56}$, final focusing, and upstream matching. The linac design [4] for this ICS suggests that a reasonable range for $M_{56}$ tuning is 1.5-2.0 m.

A typical S-shaped chicane compressor ensures achromaticity by having a net horizontal phase advance of $2 \pi$, with a pair of symmetrically-placed horizontal focusing quadrupoles to tune achromaticity and a vertical focusing quadrupole at the center symmetry point to control vertical betas. Here the compact design pushes us towards stronger focusing, and we investigate two designs: one with net $3 \pi$ phase advance and a net $90^{\circ}$ bend for corner use, and one with net $4 \pi$ phase advance for larger dispersion and $M_{56}$ lever arm. $M_{56}$ is tunable for both designs by adjusting the center symmetric bend angles and re-matching achromaticity and final focus.

\subsection{DESIGNS AND RESULTS}

The 6-D particle tracking code Elegant [22], was used for lattice design, optimization, and particle tracking, including simulation of short quadrupole end effects [23] as described in the next section. Each lattice has three quadrupoles in two symmetric families between the center dipoles. For a given central dipole bend angle (and hence $M_{56}$ ),

- The pair of symmetric quadrupoles are tuned to make the compressor achromatic.

- The center quadrupole is tuned to control vertical beam size entering the final focus section.

- The final focus is re-tuned to match collision IP conditions of $\beta_{x, y}^{*}=5 \mathrm{~mm}$ and $\alpha_{x, y}^{*}=0$.

In both designs, peak beam sizes are well-controlled, and chromatic effects of the final focus are minimal, indicating no need for nonlinear corrections that would otherwise distort the transverse phase space. The next step of the process is to include sextupoles at high dispersion locations in the bunch compressor to control the longitudinal distribution and compression linearity [24]. 


\subsection{1 $3 \pi$ COMPRESSOR}

The first compressor, denoted " $3 \pi$ ", has a total dispersion phase advance of $3 \pi$ and a net bending of $90^{\circ}$, making it suitable for a compact corner design. Four quadrupoles are used in the final focus. The floor layout and the transverse optics are shown in Fig. 21, and relevant parameters are shown in Table 5. The compressor fits in a $3.5 \times 4 \mathrm{~m}$ area, and in these conditions is tuned for $M_{56}=1.498 \mathrm{~m}$. Note that for this compressor, the first and fourth dipoles bend in the same direction to provide the dispersion cancellation and overall bend.

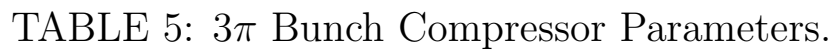

\begin{tabular}{lcc}
\hline \hline Parameter & Value & Units \\
\hline Final $\beta_{x}, \beta_{y}$ & 0.005 & $\mathrm{~m}$ \\
Final $\alpha_{x}, \alpha_{y}$ & $<10^{-8}$ & - \\
Maximum $\beta$ & $\approx 85.8$ & $\mathrm{~m}$ \\
$M_{56}$ & 1.498 & $\mathrm{~m}$ \\
Floor Dimensions & $3 \times 4$ & $\mathrm{~m}$ \\
Dipole Lengths & $0.8,1.0,0.8,1.0$ & $\mathrm{~m}$ \\
Dipole Bend Angles & $70,-115,-115,70$ & $\circ$ \\
Max Quadrupole Gradient & $\approx 9.5$ & $\mathrm{~T} / \mathrm{m}$ \\
Quadrupole Length & 0.1 & $\mathrm{~m}$ \\
\hline \hline
\end{tabular}



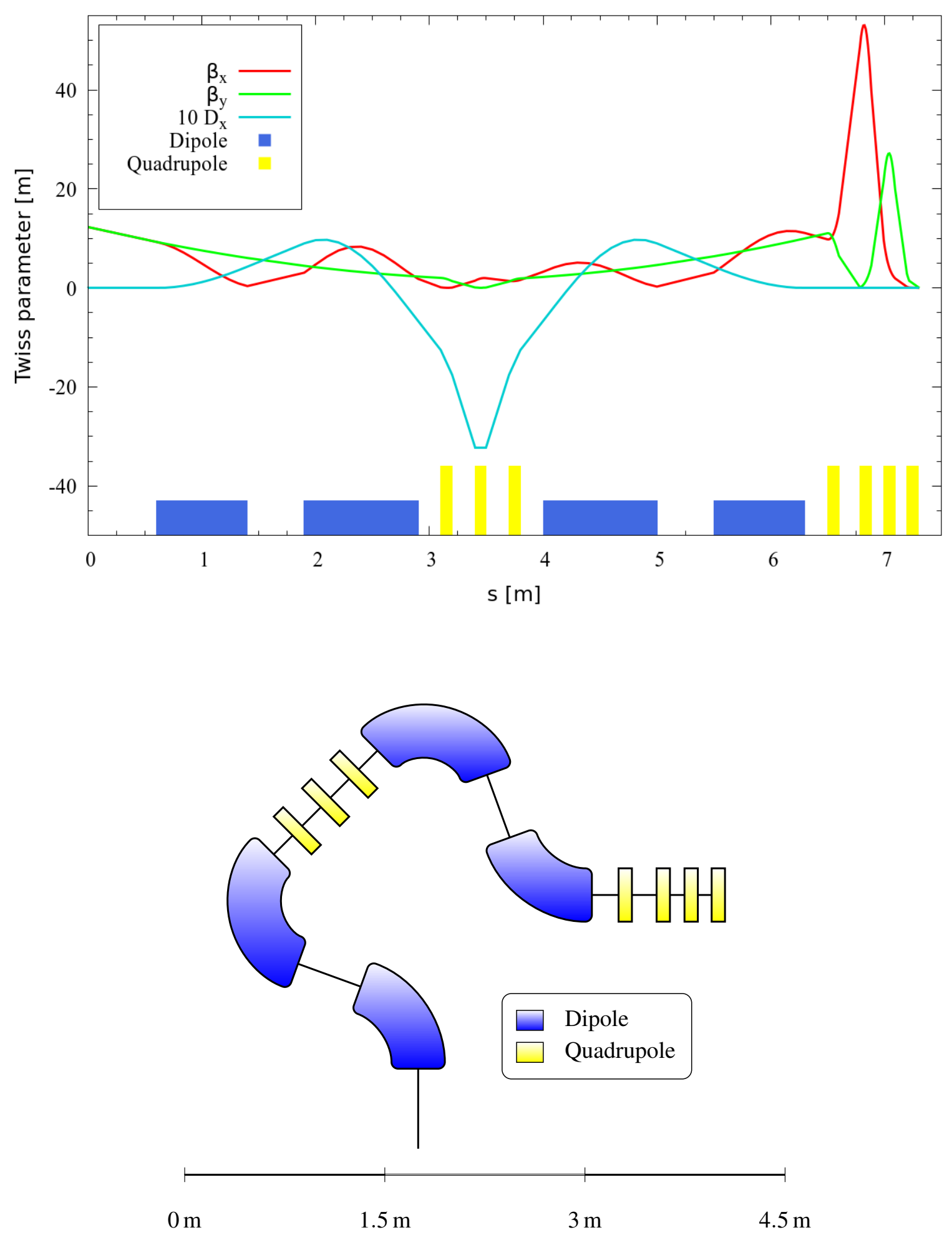

FIG. 21: $3 \pi$ bunch compressor optics and floor plan. 


\subsection{2 $4 \pi$ COMPRESSOR}

The second compressor, denoted " $4 \pi$ ", has a total dispersion phase advance of $4 \pi$ and no net bending; it is more transversely compact than the $3 \pi$ design. three quadrupoles are used in the final focus. The floor layout and the transverse optics are shown in Fig. 22 and relevant parameters are shown in Table 6. The compressor fits in a $2.0 \times 4 \mathrm{~m}$ area, and in these conditions is tuned for $M_{56}=1.4 \mathrm{~m}$. Additional quadrupoles may be added in the outside drifts for extra optics control.

TABLE 6: $4 \pi$ Bunch Compressor Parameters.

\begin{tabular}{lcc}
\hline \hline Parameter & Value & Units \\
\hline Final $\beta_{x}, \beta_{y}$ & 0.005 & $\mathrm{~m}$ \\
Final $\alpha_{x}, \alpha_{y}$ & $<10^{-8}$ & - \\
Maximum $\beta$ & $\approx 29.5$ & $\mathrm{~m}$ \\
$M_{56}$ & 1.4 & $\mathrm{~m}$ \\
Floor Dimensions & $2 \times 4.3$ & $\mathrm{~m}$ \\
Dipole Lengths & $0.35,1.134,1.134,0.35$ & $\mathrm{~m}$ \\
Dipole Bend Angles & $40,-175,175,-40$ & $\circ$ \\
Max Quadrupole Gradient & $\approx 9.15$ & $\mathrm{~T} / \mathrm{m}$ \\
Quadrupole Length & 0.1 & $\mathrm{~m}$ \\
\hline \hline
\end{tabular}



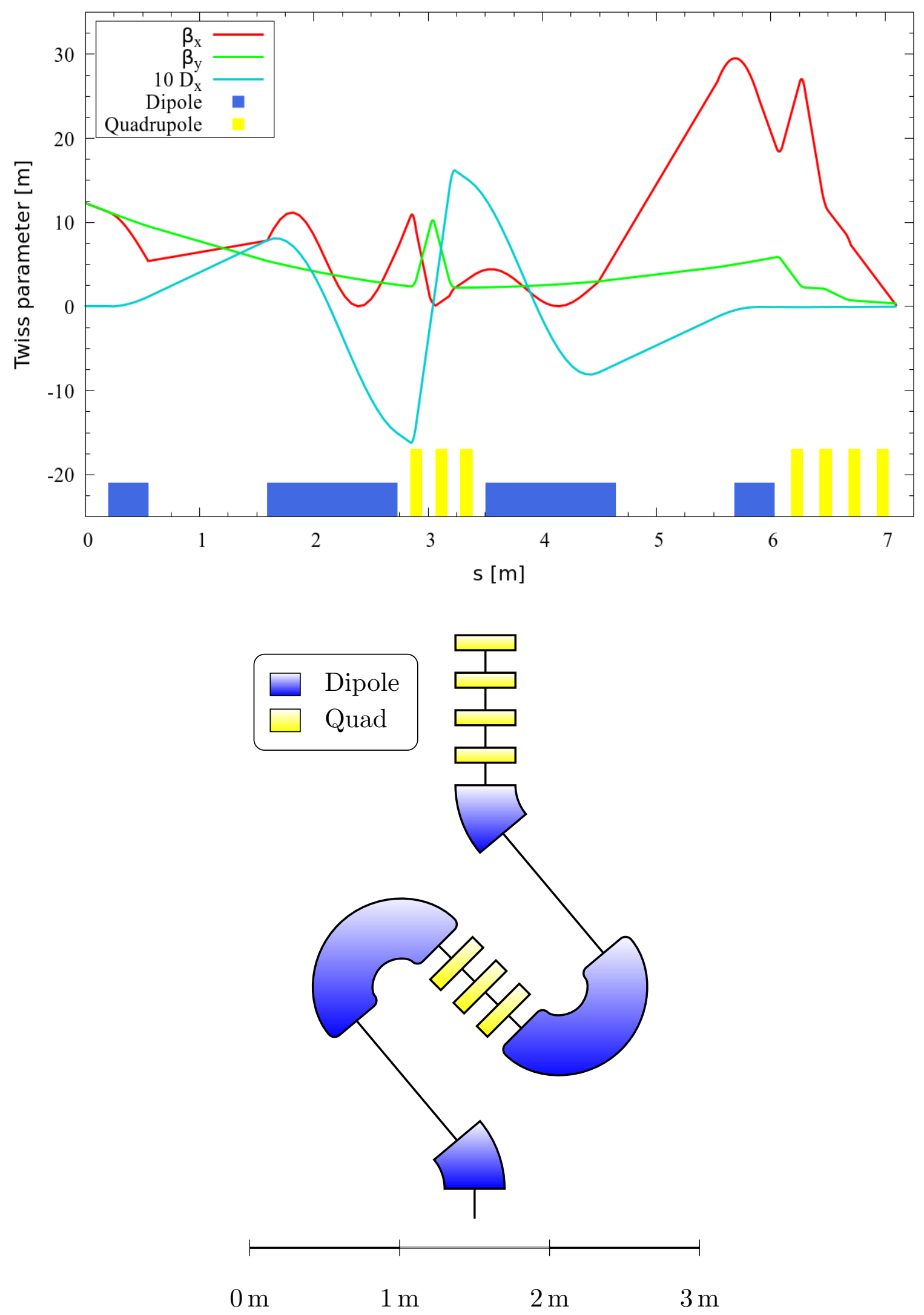

FIG. 22: $4 \pi$ bunch compressor optics and floor plan. 


\subsection{QUADRUPOLE CONSIDERATIONS}

The quadrupoles in both designs are typically $10 \mathrm{~cm}$ long with an exception of the focusing quartet of $4 \pi$ compressor, which are $15 \mathrm{~cm}$ long, with a maximum field gradient of nearly $10 \mathrm{~T} / \mathrm{m}$. Some parameters for various quadrupoles used in these design are shown in Table 7 .

With the design beam pipe diameter, these parameters match those of commercially available quadrupoles such as those from RadiaBeam. The air-cooled diamond type quadrupole BEMQD-01-155-245 [25] is such a magnet which is close to the required dimensions while having a max gradient of $12 \mathrm{~T} / \mathrm{m}$.

TABLE 7: Selected data for the quadrupoles used in the design.

\begin{tabular}{lcc}
\hline \hline Parameter & Value(range) & Units \\
\hline Bore & 0.04 & $\mathrm{~m}$ \\
Magnetic field gradient & $2.5-9.7$ & $\mathrm{~T} / \mathrm{m}$ \\
Length & 10 & $\mathrm{~cm}$ \\
Total current (NI) & $434-1684$ & $\mathrm{~A}$ \\
Conductor cross-section & $4.3-16.84$ & $\mathrm{~cm}^{2}$ \\
(air-cooled) & & \\
Conductor cross-section & $0.43-1.684$ & $\mathrm{~cm}^{2}$ \\
(water-cooled) & & \\
\hline \hline
\end{tabular}

TABLE 8: RadiaBeam Diamond Quadrupole.

\begin{tabular}{lcc}
\hline \hline Parameter & Value & Units \\
\hline Bore & 0.0394 & $\mathrm{~m}$ \\
Max field gradient & 12 & $\mathrm{~T} / \mathrm{m}$ \\
Magnetic length & $\sim 9.74$ & $\mathrm{~cm}$ \\
Total current (NI) & 1940.4 & A-turns \\
\hline \hline
\end{tabular}


Fringe field from quadrupoles [26] are sometimes neglected in designs due to having a magnet length far longer than the extension of the fringe field. Since the two bunch compressor designs use quadrupoles that have a length comparable to the fringe fields, we investigated the effect of inclusion of fringe fields on the transport and matching. Considering the fringe field to be symmetric on both ends, a single parameter Enge function [27] was used to calculate the required integrals for elegant to calculate the final beta functions with effects from the fringe fields from the quadrupoles.

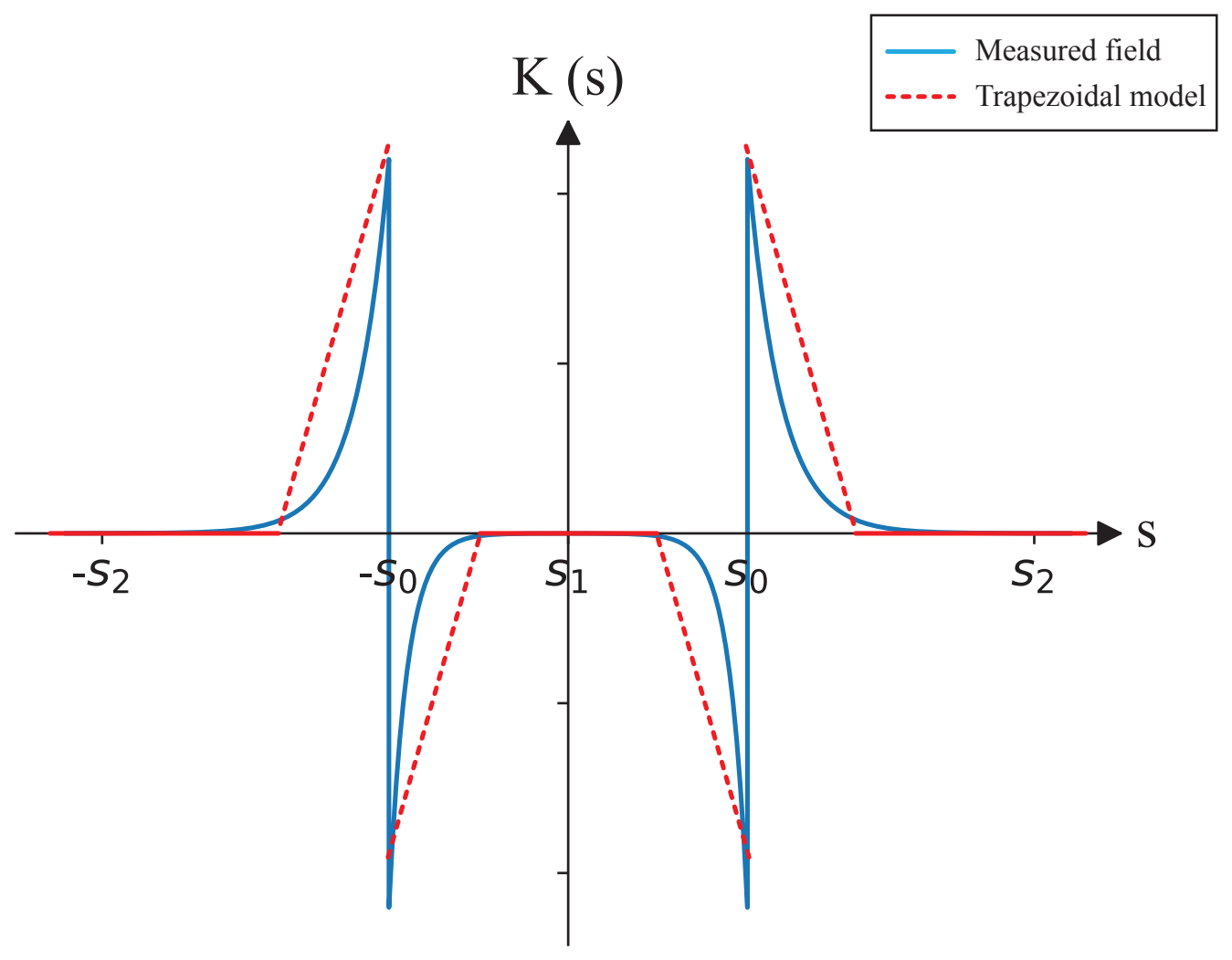

FIG. 23: Typical fringe field distribution from field measurements and the corresponding trapezoidal model.

The total linear fringe map [23] is denoted by

$$
R_{f}=e^{: f_{2}^{-}}: e^{: f_{2}^{+}:}=e^{: f_{2}:}
$$


where

$$
\begin{aligned}
f_{2} \cong f_{2}^{-} & +f_{2}^{+}+\frac{1}{2}\left[f_{2}^{-}, f_{2}^{+}\right] \\
\approx- & \left(I_{1}^{-}+I_{1}^{+}\right)\left(x p_{x}-y p_{y}\right)-\frac{I_{2}^{-}+I_{2}^{+}}{2}\left(p_{x}^{2}-p_{y}^{2}\right)+\frac{K_{0} I_{2}^{-}}{2}\left(x^{2}+y^{2}\right) \\
& +\frac{2 K_{0} I_{3}^{-}}{3}\left(x p_{x}+y p_{y}\right)+\frac{\Lambda_{2}^{-}+\Lambda_{2}^{+}}{2}\left(x^{2}+y^{2}\right)-\frac{1}{2} I_{0}^{+}\left(I_{1}^{-}+I_{1}^{+}\right)\left(x^{2}+y^{2}\right) \\
& -\frac{1}{2} I_{0}^{+}\left(I_{2}^{-}+I_{2}^{+}\right)\left(x p_{x}+y p_{y}\right)+\cdots .
\end{aligned}
$$

Using the field profile shown in Fig. 24 we found the Enge coefficient $a_{1}=4.6$.

Since the Radiabeam qudrupole matches well with the quadrupoles used in the design, we used the end magnetic field profile for the Radiabeam quadrupole as shown in Fig. 24. and fit the data to a one-parameter Enge function, where $s$ is the axis along the center and $D$ is the bore diameter of the quadrupole.

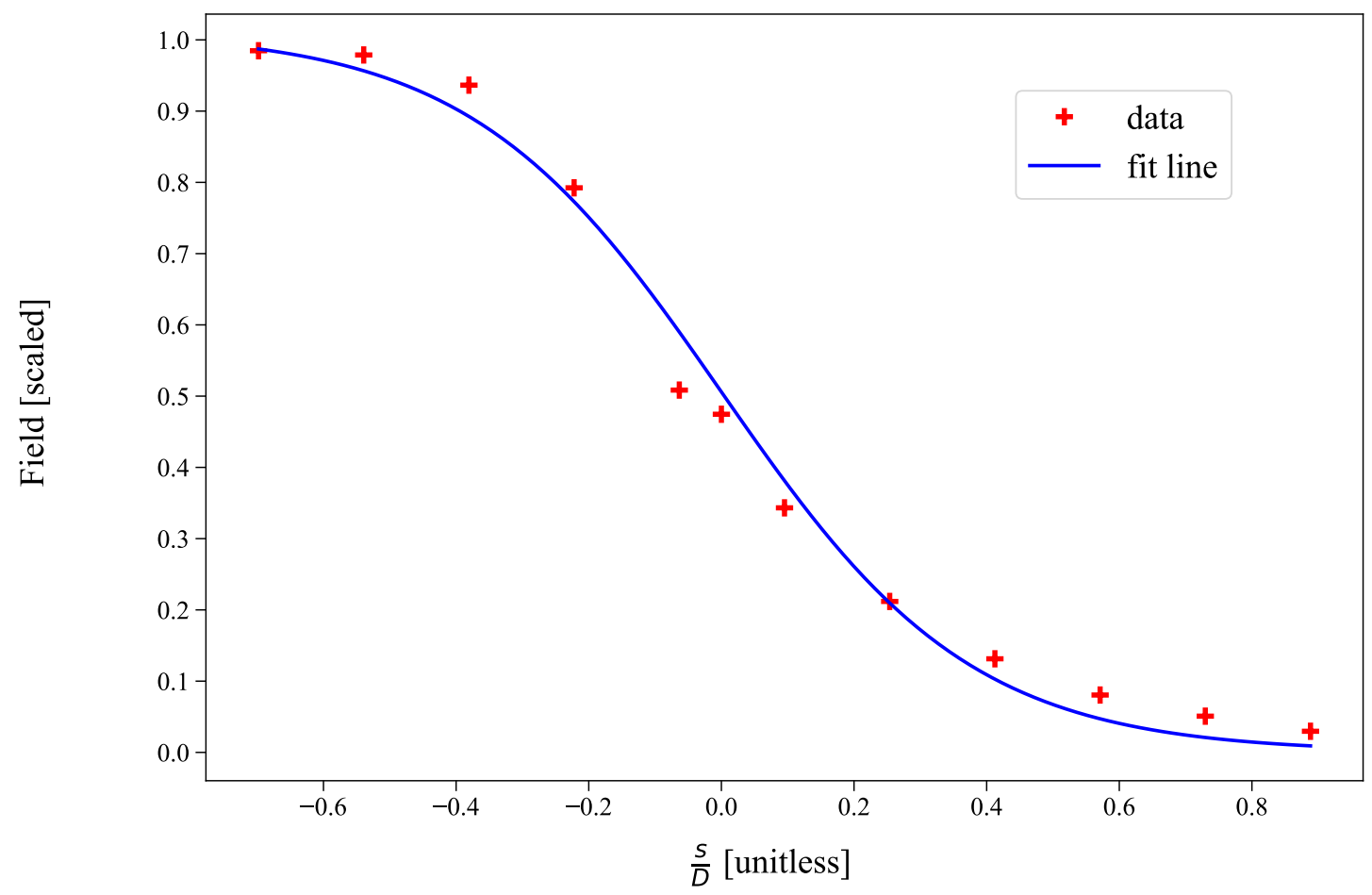

FIG. 24: Fit of normalized fringe field for Radiabeam diamond quadrupole, consistent with these designs, showing fit to one-parameter Enge function.

The one-parameter Enge function fit is:

$$
E(s)=\frac{1}{1+\exp \left(-a_{1} s / D\right)}=\frac{1+\tanh \left(a_{1} s / 2 D\right)}{2} .
$$


This gives the Enge coefficient $a_{1}=5.42$. This can be used to get the quadrupole fringe field strength along the axis [27] by using

$$
k(s)=\frac{k_{0}}{2}\left[\tanh \left(a_{1}\left(\frac{L}{2}+s\right)\right)+\tanh \left(a_{1}\left(\frac{L}{2}-s\right)\right)\right] .
$$

With this, the input values to be used in ELEGANT were calculated using the integrals in Eq. 75 .

$$
\begin{aligned}
i_{0}^{-} & =\int_{s 1}^{s 0} \tilde{k}(s) d s & i_{0}^{+} & =\int_{s 0}^{s 2} \tilde{k}(s) d s \\
i_{1}^{-} & =\int_{s 1}^{s 0} \tilde{k}(s)\left(s-s_{0}\right) d s & i_{1}^{+} & =\int_{s 0}^{s 2} \tilde{k}(s)\left(s-s_{0}\right) d s \\
i_{2}^{-} & =\int_{s 1}^{s 0} \tilde{k}(s)\left(s-s_{0}\right)^{2} d s & i_{2}^{+} & =\int_{s 0}^{s 2} \tilde{k}(s)\left(s-s_{0}\right)^{2} d s \\
i_{3}^{-} & =\int_{s 1}^{s 0} \tilde{k}(s)\left(s-s_{0}\right)^{3} d s & i_{3}^{+} & =\int_{s 0}^{s 2} \tilde{k}(s)\left(s-s_{0}\right)^{3} d s \\
\Lambda_{2}^{-} & =\int_{s_{0}}^{s_{2}} d s \int_{s}^{s_{0}} d s^{\prime} \tilde{k}(s) \tilde{k}\left(s^{\prime}\right)\left(s^{\prime}-s\right) & \Lambda_{2}^{+} & =\int_{s_{0}}^{s_{2}} d s \int_{s}^{s_{2}} d s^{\prime} \tilde{k}(s) \tilde{k}\left(s^{\prime}\right)\left(s^{\prime}-s\right)
\end{aligned}
$$

where $\tilde{k}(s)$ is the normalized strength of the quadrupole [23]. After the fringe fields effects was added to the lattice, a final tune was done to refit the lattice to gain the required final beam sizes.

\subsection{SECOND ORDER DISPERSION CORRECTION}

For the second order correction of the momentum compaction we added two sextupoles for each design. The general design practice is to place two sextupoles with opposite signs in a high dispersion area that is available. The highest dispersion region available was between the two central dipoles. So it was decided to put the sextupoles between the three quads in the middle. For the $4 \pi$ compressor the placement of the sextupoles was the same except for the fact that the dispersion region then has opposite signs. The length between the two middle dipoles had to be expanded to fit the two sextupoles. The length of the dipoles in $4 \pi$ design was re-calculated by keeping the term $B \rho$ constant to a value of $0.0833 \mathrm{~T}-\mathrm{m}$. Because the $3 \pi$ design has the same sign for the two large dipoles the beam size blow up inside the compressor was too high to keep the $B \rho$ constant. So in this design we made all the dipoles the same length. 
As shown in the Fig. 25 and Fig. 26, the floor plan for the $3 \pi$ and $4 \pi$ design after the addition of sextupoles did not change significantly in size from the initial design.

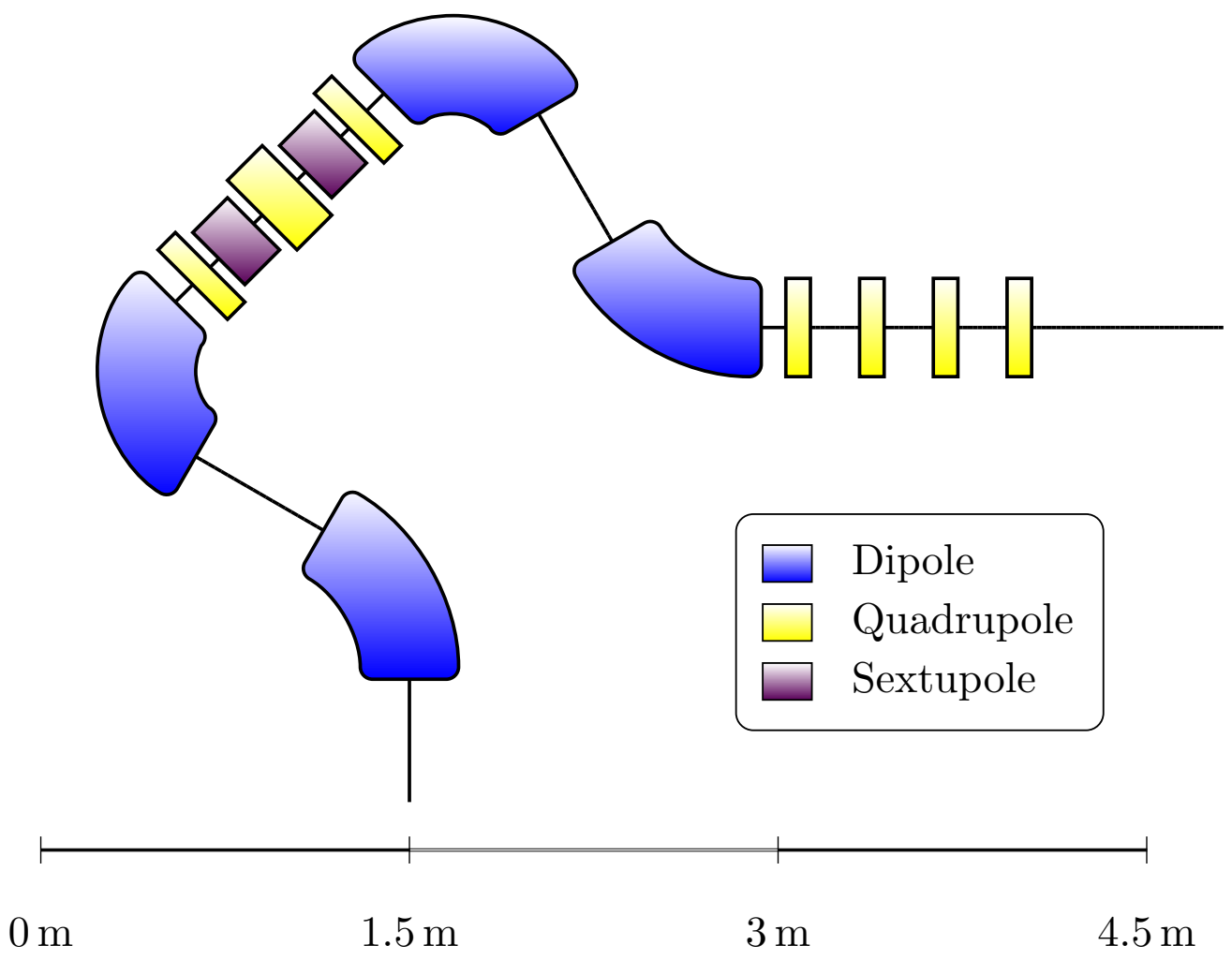

FIG. 25: $3 \pi$ design floor plan. 


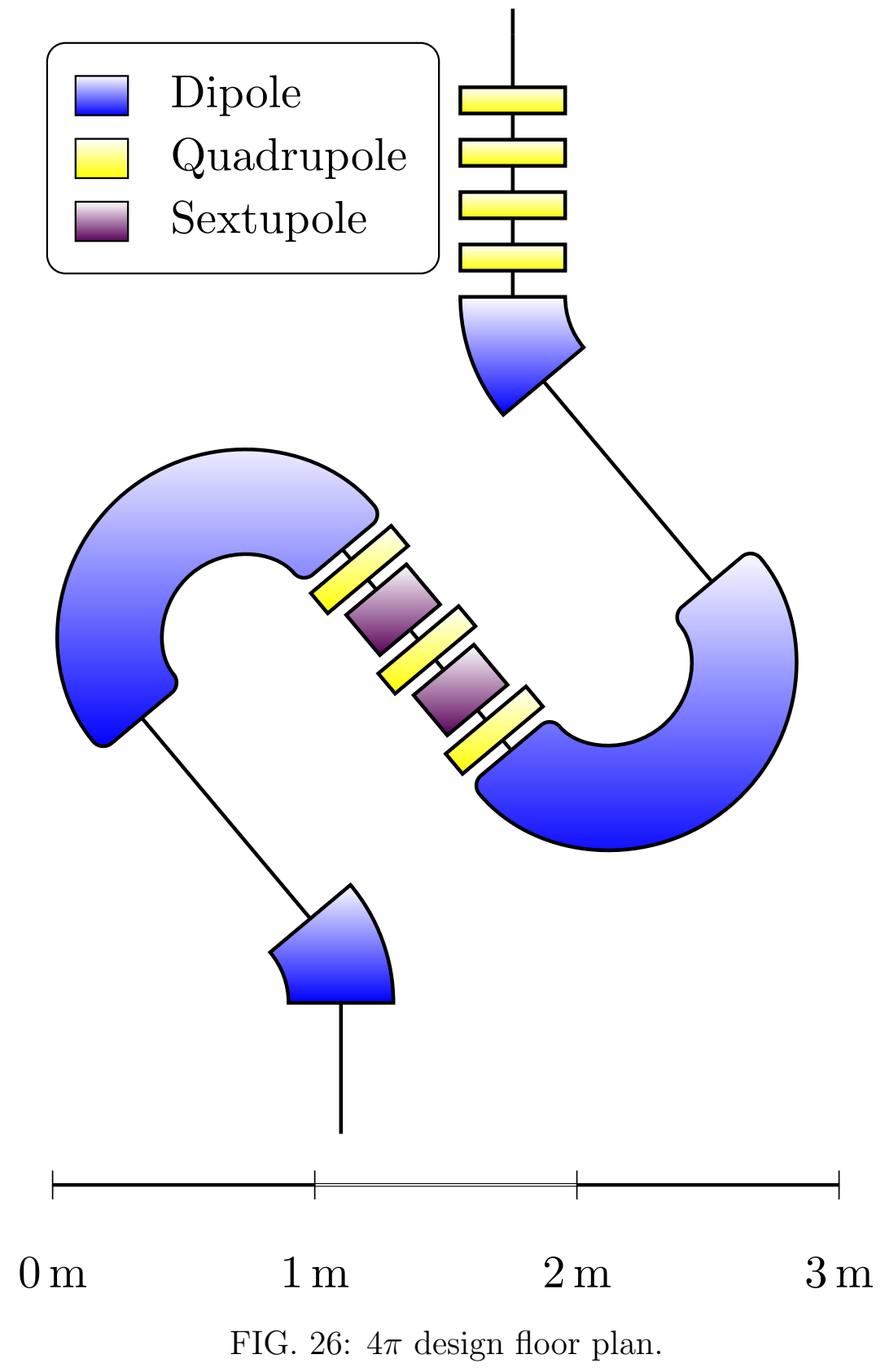


The input parameters changed from the values used in the original desgin [18]. The new new input parameters for the bunch compressor are shown in Table 9.

TABLE 9: Input beam parameters.

\begin{tabular}{lcc}
\hline \hline Parameter & Value(range) & Units \\
\hline kinetic energy & 25 & $\mathrm{MeV}$ \\
fractional momentum spread & $2.6 \mathrm{e}-03$ & - \\
$\epsilon_{x, r m s}^{N}$ & 0.16 & $\mathrm{~mm}-\mathrm{mrad}$ \\
$\epsilon_{y, r m s}^{N}$ & 0.15 & $\mathrm{~mm}-\mathrm{mrad}$ \\
$\beta_{x}$ & 82.1 & $\mathrm{~m}$ \\
$\beta_{y}$ & 75.5 & $\mathrm{~m}$ \\
$\alpha_{x}$ & 2.34 & - \\
$\alpha_{y}$ & -0.591 & - \\
$\sigma_{z}$ & 14.08 & $\mathrm{psec}$ \\
\hline \hline
\end{tabular}

With the addition of the sextupoles the optimizing scheme included the step that changed the sextupoles values to minimize the second order momentum compaction factor (alphac2 in elegant). This was done before the final optimization so that the beam is achromatic again leaving the dipole section. The output bunch parameters from the $3 \pi$ lattice is shown in Table 10. 
TABLE 10: Output beam parameters.

\begin{tabular}{lcc}
\hline \hline Parameter & Value(range) & Units \\
\hline kinetic energy & 25 & $\mathrm{MeV}$ \\
fractional momentum spread & $2.6 \mathrm{e}-03$ & - \\
$\epsilon_{x, r m s}^{N}$ & 0.16 & $\mathrm{~mm}-\mathrm{mrad}$ \\
$\epsilon_{y, r m s}^{N}$ & 0.15 & $\mathrm{~mm}-\mathrm{mrad}$ \\
$\beta_{x}$ & 0.005 & $\mathrm{~m}$ \\
$\beta_{y}$ & 0.005 & $\mathrm{~m}$ \\
$\alpha_{x}$ & $2.65 \mathrm{e}-09$ & - \\
$\alpha_{y}$ & $-5.09 \mathrm{e}-10$ & - \\
$\sigma_{z}$ & 2 & $\mathrm{psec}$ \\
\hline \hline
\end{tabular}

Fig. 27 and Fig. 28 shows the input and the output longitudinal beam profiles from the $3 \pi$ and $4 \pi$ designs respectively. The first row shows the longitudinal beam profile of the incoming bunch. The second row shows the the outgoing bunch with no second order corrections and the third shows it with second order corrections. 

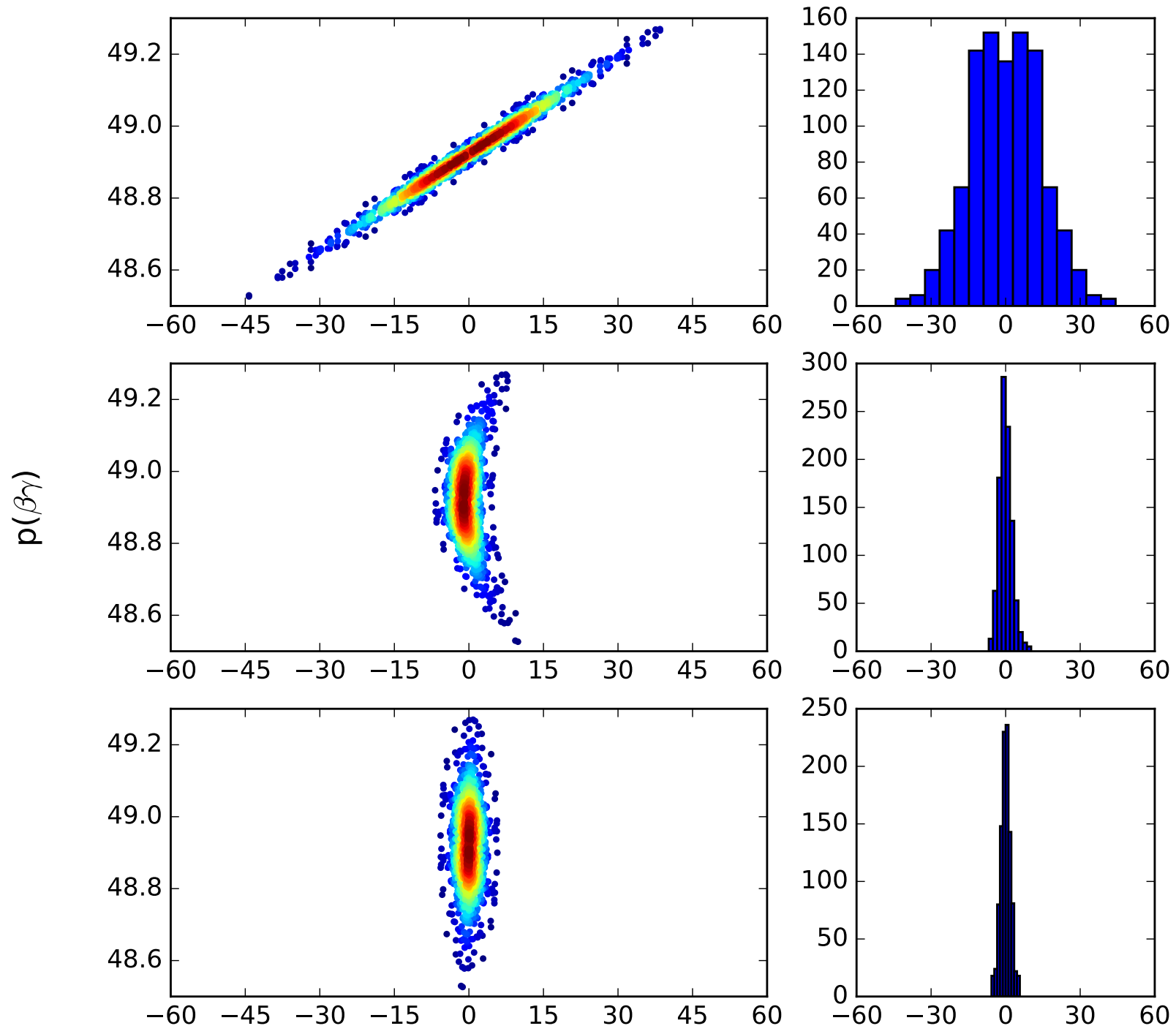

Time [ps]

FIG. 27: Longitudinal phase space (left) and time histogram (right) before compressor (top), after compressor without sextupole correction (center), and after compressor with sextupole correction (bottom) for the $3 \pi$ compressor. 

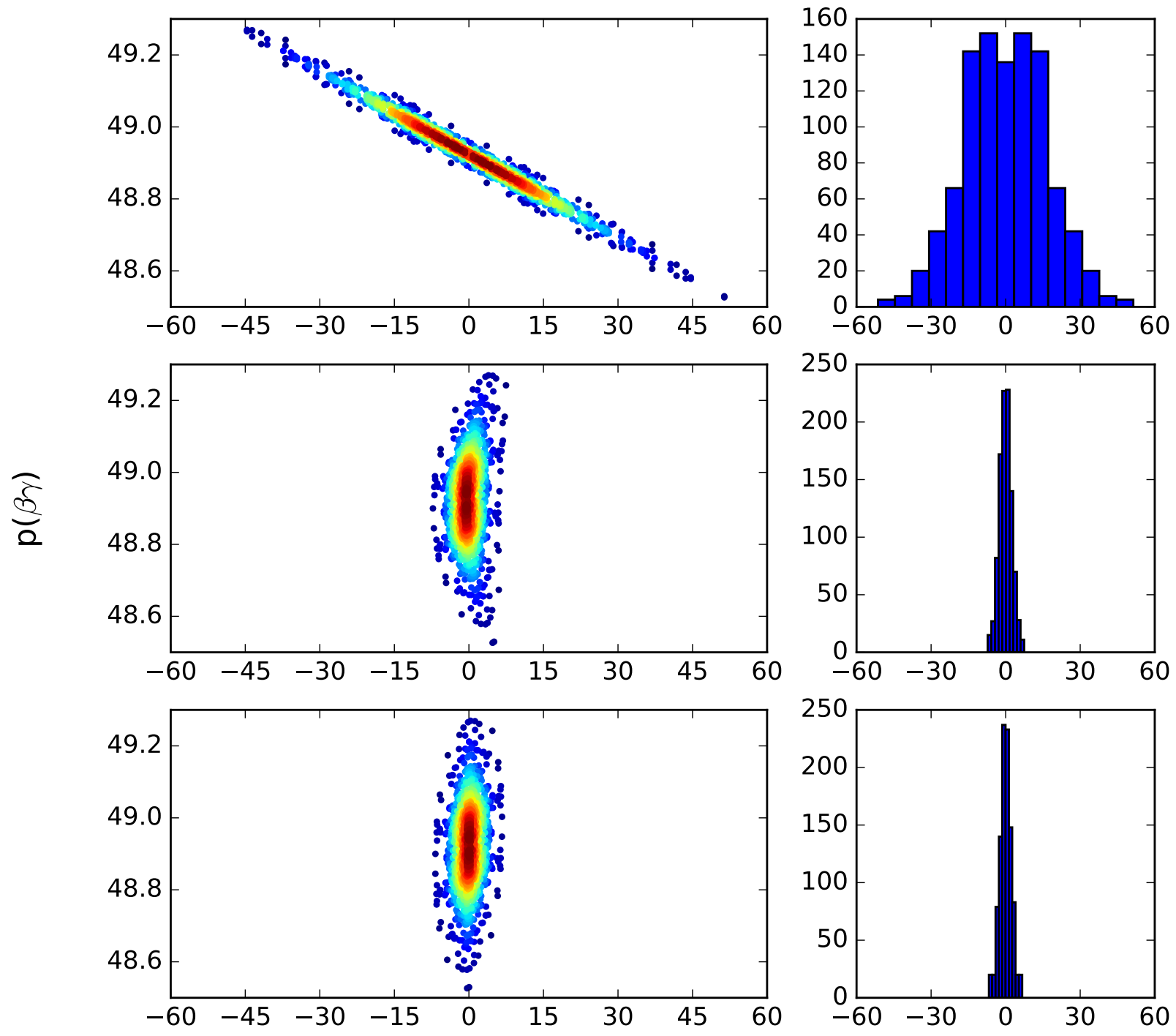

Time [ps]

FIG. 28: Longitudinal phase space (left) and time histogram (right) before compressor (top), after compressor without sextupole correction (center), and after compressor with sextupole correction (bottom) for the $4 \pi$ compressor.

The $3 \pi$ compressor was designed to be used for a positively chirped bunch while the $4 \pi$ compressor is for a negatively chirped beam. The transverse beam sizes and the horizontal dispersion function plotted along the beam elements are shown in Fig. 29 and Fig. 30. 


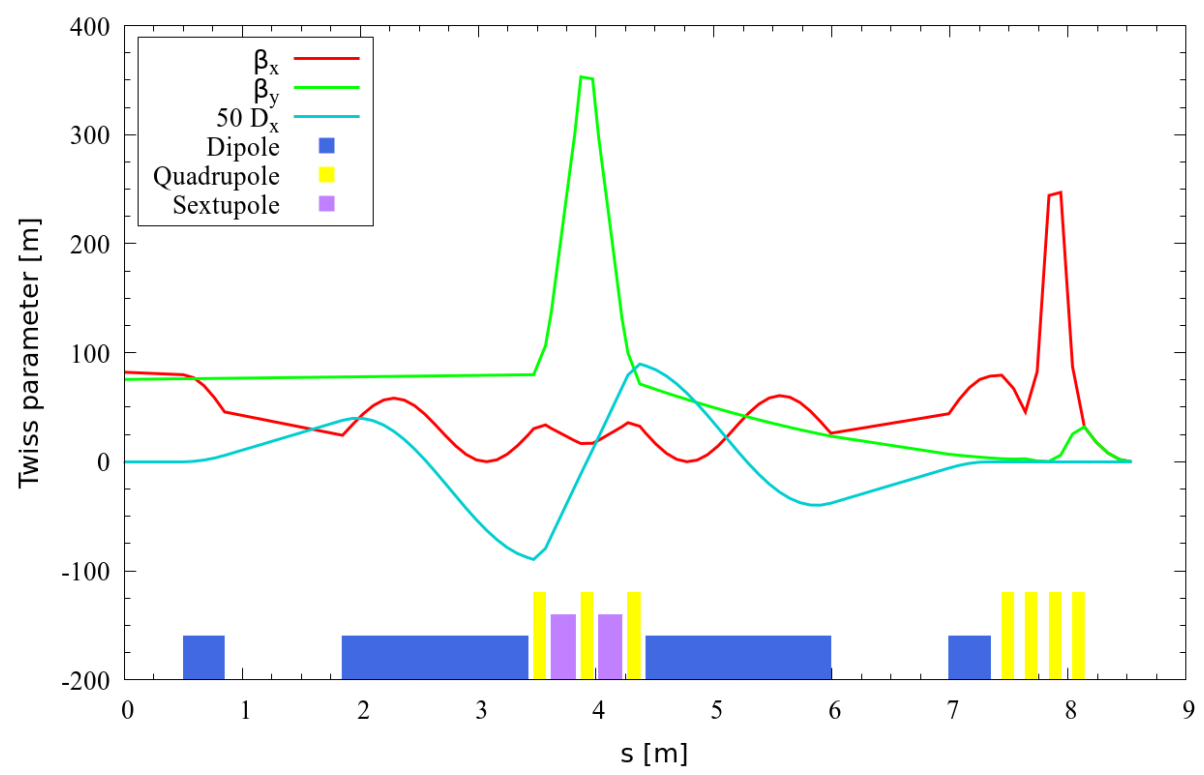

FIG. 29: $4 \pi$ beta function and dispersion.

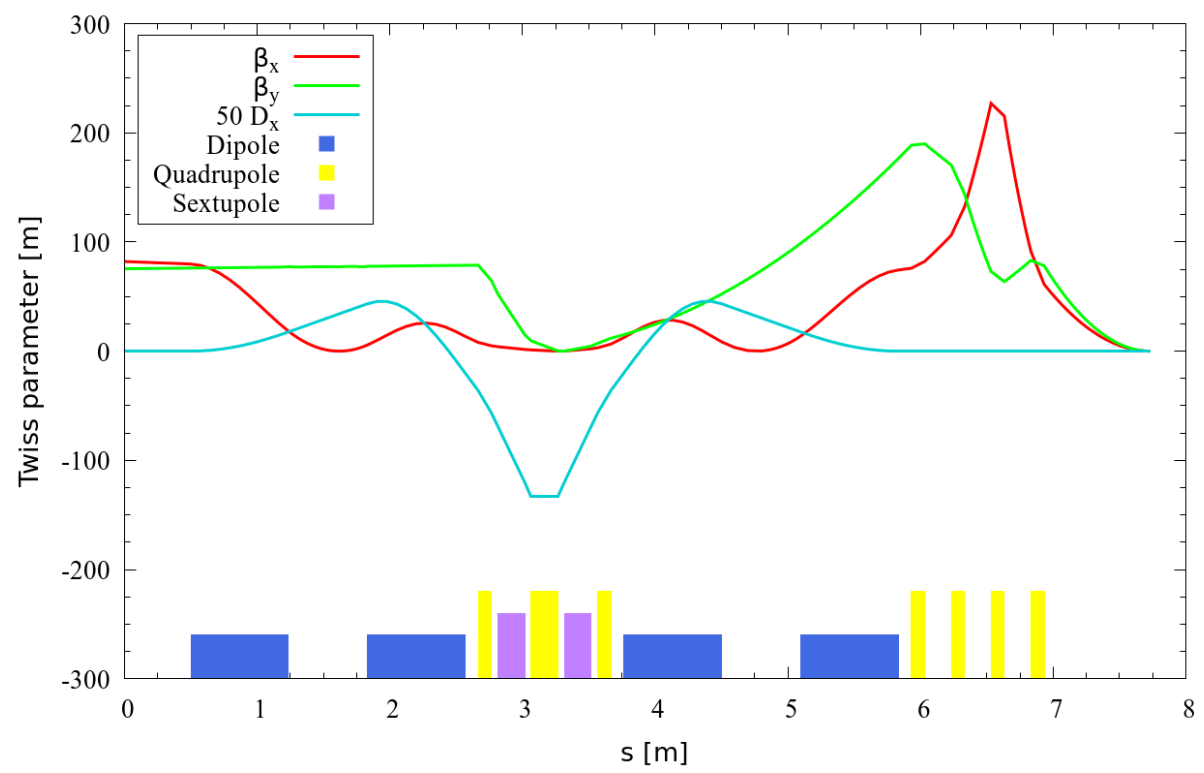

FIG. 30: $3 \pi$ beta function and dispersion.

\subsection{TUNABILITY}

A fixed momentum compaction factor results in inability to orthogonally tune the ICS on the length of the incoming bunch. For slight deviations of the length it is important to have a bunch compressor with a variable $M_{56}$. In the $4 \pi$ design, this is acheived by changing 
the bending angle of the two middle dipoles and re-optimize the compressor to achieve the required beam sizes.

TABLE 11: $M_{56}$ tunability of the $4 \pi$ compressor.

\begin{tabular}{lc}
\hline \hline Bending angle & $M_{56}$ \\
\hline 179.0 & 2.01245 \\
179.2 & 1.99265 \\
179.4 & 1.97265 \\
179.6 & 1.95243 \\
179.8 & 1.93200 \\
$\mathbf{1 8 0 . 0}$ & 1.91136 \\
180.2 & 1.89051 \\
180.4 & 1.86945 \\
180.6 & 1.84818 \\
180.8 & 1.82671 \\
181.0 & 1.80502 \\
\hline \hline
\end{tabular}

For shorter variations of the middle dipole angles the $M_{56}$ is almost linear. The tunability of the system is mainly constrained by the length between the two center dipoles and the size of the beam pipe. The length between the two center dipoles in the $4 \pi$ design is 1 meter. With a bending offset of $1^{\circ}$ in the second dipole, the offset from the center of the beam pipe at the beginning of the third dipole is then given by $L=\rho \theta=0.0174533 \mathrm{~m}$.

For $3 \pi$ design, the two center dipoles bends the same direction. So the two dipoles taken for the tunability test was the first and the thrid dipoles. Tunability of the $3 \pi$ design is considerably limited compared to the $4 \pi$ design. 
TABLE $12: M_{56}$ tunability of the $3 \pi$ compressor.

\begin{tabular}{lcc}
\hline \hline Bending angle 1st dipole & Bending angle 3rd dipole & $M_{56}$ \\
\hline 104.0 & 179.0 & -1.49793 \\
104.2 & 179.2 & -1.51906 \\
104.4 & 179.4 & -1.54034 \\
104.6 & 179.6 & -1.56178 \\
104.8 & 179.8 & -1.58338 \\
$\mathbf{1 0 5 . 0}$ & $\mathbf{1 8 0 . 0}$ & -1.60513 \\
105.2 & 180.2 & -1.62703 \\
105.4 & 180.4 & -1.64908 \\
105.6 & 180.6 & -1.67128 \\
105.8 & 180.8 & -1.69364 \\
106.0 & 181.0 & -1.71614 \\
\hline \hline
\end{tabular}




\section{CHAPTER 5}

\section{DEBUNCH/REBUNCH}

\subsection{BACKGROUND}

The first method that was considered for the final ion bunch formation for the JLEIC ion collider ring is the debunch/rebunch method. Unlike the other method mentioned in this thesis, debunch/rebunch has two advantages: the final harmonic can be arbitrary (even prime), and only two RF system frequencies are required. After injection and acceleration of the initial number of bunches, the RF that keeps the beam bunched is adiabatically ramped down to zero. At this point each bunch longitudinally drifts with no RF control, with higher momentum particles going forward and lower momentum particles trailing behind. Over time this difference becomes larger, and the whole circumference of the collider ring becomes filled with particles. Longitudinal emittance is conserved if the process is adiabatic. A larger distribution of the particles in time necessarily means a smaller distribution in momentum spread or energy spread compared to the initial bunch. With the beam at this point a fully coasting beam, the final RF is ramped up to capture the beam into the final number of bunches.

In JLEIC this means injecting 26 bunches at harmonic 28, accelerating them to higher energy, then debunching and rebunching to the final number of bunches of 3328 at harmonic 3584. Although this method is commonly used, and requires fewer RF frequencies compared to adiabatic bunch splitting, it has significant drawbacks. While coasting, the beam has very small momentum spread compared to the initial bunch, and it becomes more susceptible to longitudinal instabilities, known as microwave instabilities, that can quickly cause the beam's longitudinal emittance to grow. Also at this point the beam is drifting in space with no RF control so no energy losses due to impedances (such as resistive wall) can be recovered.

The Fermilab Tevatron proton-antiproton collider included a debuncher ring [28], [29], where the momentum spread of the antiproton bunches was reduced before stacking and use in the collider. Bunches entered the debuncher ring retaining a $53 \mathrm{MHz}$ structure from earlier RF. They were then intentionally mismatched to the debuncher RF bucket at injection into the debuncher. In one quarter of a synchrotron period, the injected bunch rotated to 
increase the bunch length and decrease the momentum spread. Finally, the RF voltage was adiabatically ramped down to create a nearly coasting beam with much smaller momentum spread.

The ALPHA project [30] at Indiana University Center for Exploration of Energy and Matter [31] was designed to test radiation effects on electronic chips and was also to be used as a compact high brightness X-ray source. For this purpose the electron bunches injected were debunched in one revolution to produce a long coasting beam [32].

JLEIC design requirements include abort gaps to accommodate an abort kicker rise time. A pure coasting beam leaves no empty space in the ring for an abort gap, so complete debunching is not appropriate for JLEIC. Introducing barrier buckets to preserve abort gaps addresses this requirement and may make debunch/rebunch a viable option.

Using the parameters from Table 1 and Table 3 the $95 \%$ longitudinal emittance of each bunch at collision can be calculated from the Eq. 42 to be $0.0183 \mathrm{eV}$-s. Assuming a completely adiabatic bunch formation process, the emittance for one bunch just after injection (at $\mathrm{h}=28$ ) can be calculated to be $2.345 \mathrm{eV}$-s. For 26 bunches the total longitudinal emittance is then $60.98 \mathrm{eV}$-s. For the case of a complete debunching scheme without a barrier bucket, the beam is spread to the entire circumference $\left(T_{\text {rev }}=7.185 \mu \mathrm{s}\right)$ which gives a beam energy spread $\sigma_{E}=1.5 \mathrm{MeV}$. For the scheme with barrier buckets this gives an energy spread $\sigma_{E}=1.62 \mathrm{MeV}$. At $100 \mathrm{GeV}$ this gives an energy spread of $d E / E=1.5 \times 10^{-5} \mathrm{MeV}$ and $d E / E=1.62 \times 10^{-5} \mathrm{MeV}$ respectively.

This essentially brings up the question of microwave instability that may occur from the broad band impedance. The Keil-Schnell criterion was used to obtain an impedance threshold to compare with the current longitudinal impedance estimations for the JLEIC ion collider ring.

The debunch/rebunch method requires considerably fewer RF systems compared to bunch splitting discussed in the following chapters. However, the debunched beam has small momentum spread that may suffer from microwave instability that would result in an emittance growth larger than the JLEIC tolerance. The theory behind barrier bucket dynamics and an impedance threshold for coasting beams are discussed in the following sections.

\subsection{THEORY}

\subsubsection{BARRIER BUCKET DYNAMICS}

A barrier bucket is a single-period RF waveform (typically a sinusoidal or rectangular 
wave) that acts as a barrier for particles that are otherwise coasting in a synchrotron. Particles with momentum offset from the synchronous particles will continue to slip until they encounter the barrier waveform. At that point they encounter a restoring force that turns them in the opposite direction, resulting in a change to the slip direction. For a particle trapped between two barrier pulses, this results in a synchrotron oscillation, though the period of this synchrotron oscillation is quite large. The barrier bucket timing can be changed while particles are trapped, manipulating the length and corresponding momentum spread of the beam. Barrier buckets are considered here for JLEIC to see if coasting beam instabilities can be avoided, and necessary abort gaps can be maintained, while still solving the bunch formation problem using the debunch/rebunch method.

Starting from the initial bunch configuration, barrier buckets are created within the two $h=28$ abort gaps. With the bunches now constrained in two groups of 13 bunches, the beam is adiabatically debunched, then rebunched to the final number of bunches while maintaining the abort gaps. This is schematically shown in Fig. 31. The longitudinal phase space distribution of a beam trapped between two barrier buckets is shown in Fig. 32 .

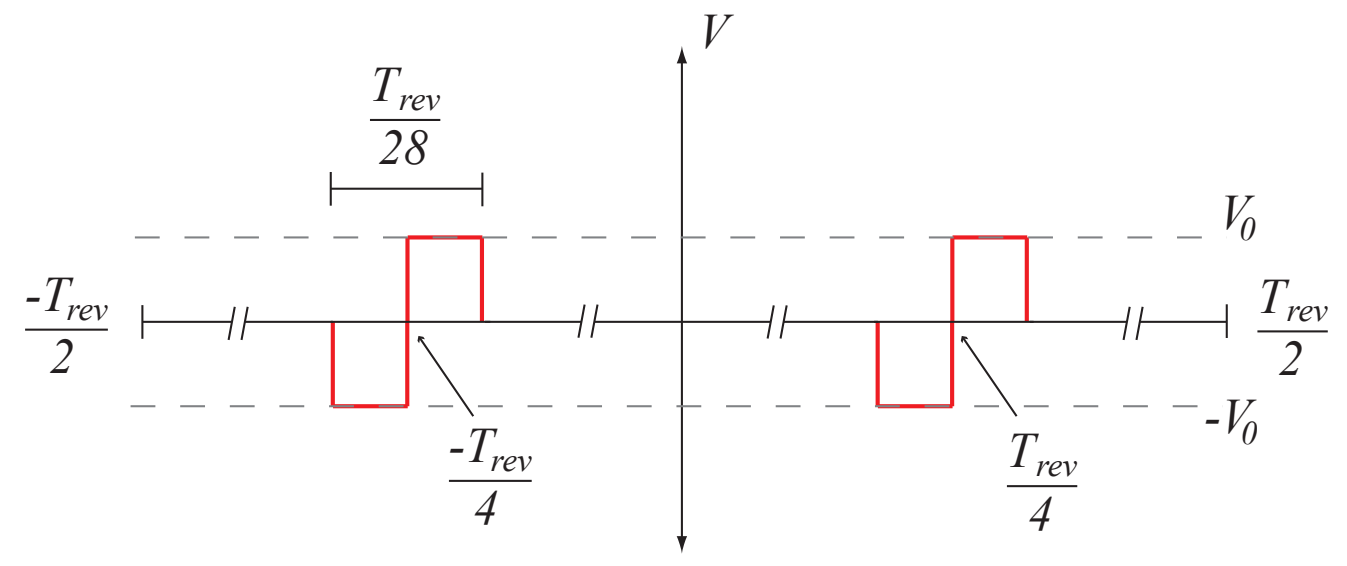

FIG. 31: Barrier bucket form for JLEIC debunch/rebunch bunch formation. Barrier buckets are created in the locations of the two $h=28$ abort gaps, dividing the beam in to two equal sections each containing 13 bunches at $h=28$. 


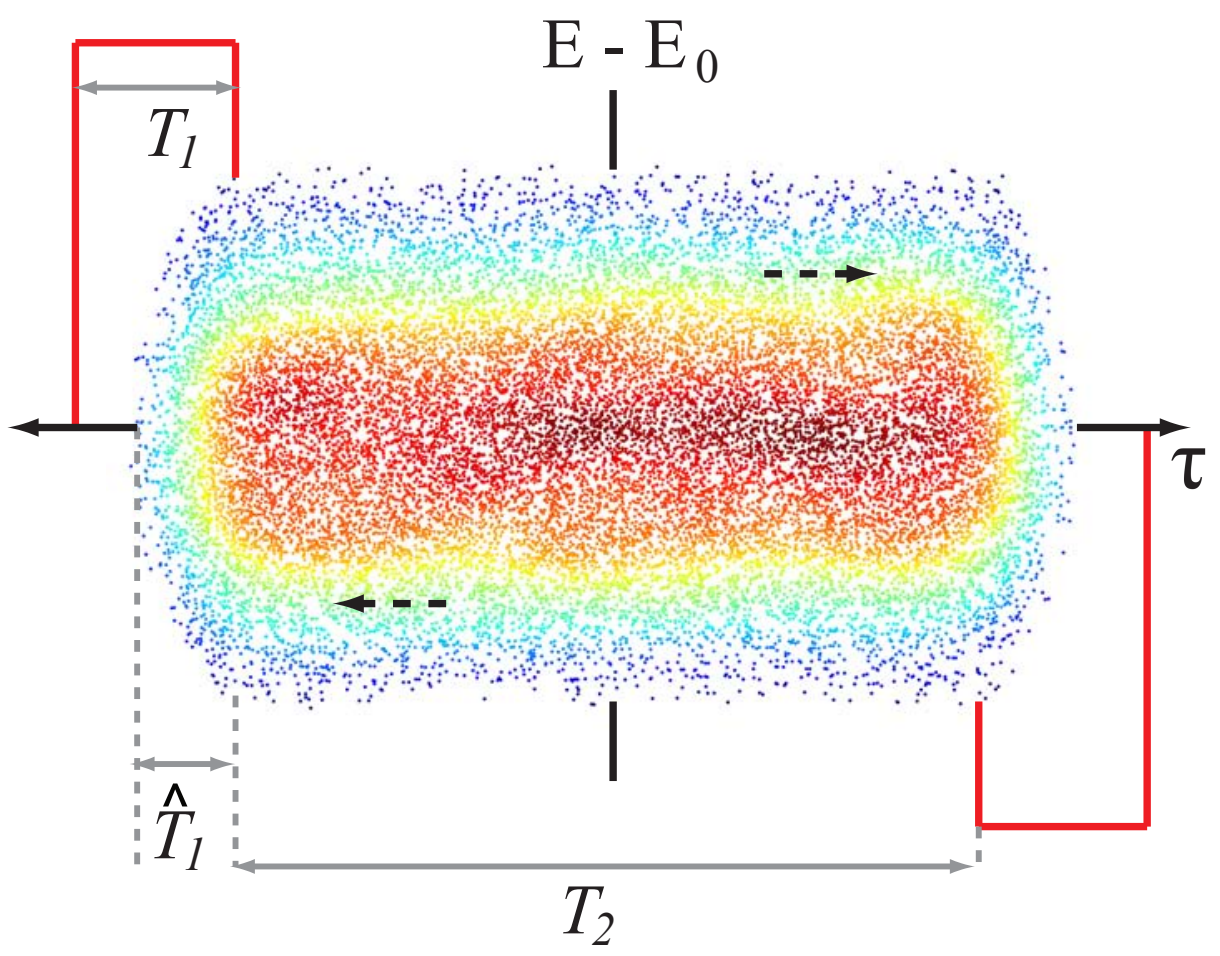

FIG. 32: A phase space distribution of a bunch insider a barrier bucket. $T_{1}$ is half the period of the barrier bucket, $T_{2}$ is the length of time between barrier buckets, and $\hat{T}_{1}$ is the extent to which a given bunch distribution penetrates the barrier bucket potential.

Re-writing the Hamiltonian for the longitudinal motion of a particle with a voltage function that represents a barrier bucket [33]

$$
H(\tau, \Delta E)=-\frac{1}{2} \frac{\eta}{\beta^{2} E_{0}}(\Delta E)^{2}-\frac{e}{T_{\mathrm{rev}}} \int_{0}^{\tau} V(s) d s,
$$

where $\tau$ is the time difference between the test particle and the design particle passing the same point of the beam pipe in one revolution. It replaces the phase coordinate used in the Hamiltonian defined in section 3.6. The voltage $V(s)$ here is defined by the shape and duration of the barrier pulse. The bucket height is then derived from the Hamiltonian at the separatrix

$$
\begin{aligned}
H_{\text {bucket }} & =-\frac{1}{2} \frac{\eta}{\beta^{2} E_{0}}\left(\Delta E_{\text {bucket }}\right)^{2}=\frac{e}{T_{\text {rev }}} \int_{0}^{\tau} V(s) d s \\
\Rightarrow \Delta E_{\text {bucket }} & =\left[\frac{2 \beta^{2} E_{0}}{T_{\text {rev }}}\left|\frac{1}{\eta} \int_{\frac{T_{2}}{2}}^{\frac{T_{2}}{2}+T_{1}} \mathrm{eV}(s) d s\right|\right]^{\frac{1}{2}} .
\end{aligned}
$$


The particles that are inside the separatrix move in phase with no change in their energy deviation until they arrive at the barrier pulse. At this point the particle's momentum offset will change until it reverses in sign. During this transformation the particles penetrate the barrier pulse with the penetration depth $\left(\hat{T}_{1}\right)$ which relates to the maximum energy deviation of the particle. Eq. 77 can be changed to find a relation between the maximum energy deviation and $\hat{T}_{1}$

$$
\Delta \hat{E}=\left[\frac{2 \beta^{2} E_{0}}{T_{\mathrm{rev}}}\left|\frac{1}{\eta} \int_{\frac{T_{2}}{2}}^{\frac{T_{2}}{2}+\hat{T}_{1}} \mathrm{eV}(s) d s\right|\right]^{\frac{1}{2}}
$$

between the two barrier pulse $\left(-T_{2} / 2 \leq \tau \leq T_{2} / 2\right)$ this energy deviation remains constant. However, inside the bucket the energy deviation changes which can be represented by

$$
\Delta E(\tau)=\left[\frac{2 \beta^{2} E_{0}}{|\eta| T_{\text {rev }}} \int_{\frac{T_{2}}{2}+\tau}^{\frac{T_{2}}{2}+\hat{T}_{1}}\right] .
$$

Applying this to the equation of motion $d \tau / d t=-\eta\left(\Delta E / \beta^{2} E_{0}\right)$ and integrating both sides will give the synchrotron period

$$
T_{\mathrm{s}}=\frac{\beta^{2} E_{0}}{|\eta|}\left(\frac{2 T_{2}}{\Delta \hat{E}}+4 \int_{0}^{\hat{T}_{1}} \frac{d \tau}{\Delta E(\tau)}\right)
$$

which contains two parts, with the first part representing the motion between the two barrier pulses and the second part representing the motion inside the barrier pulse. For a rectangular barrier pulse the voltage can be defined as

$$
V(\tau)= \begin{cases}-V_{0} & -\left(\frac{1}{2} T_{2}+T_{1}\right) \leq \tau \leq-\frac{1}{2} T_{2} \\ 0 & -\frac{1}{2} T_{2} \leq \tau \leq \frac{1}{2} T_{2} \\ V_{0} & \frac{1}{2} T_{2} \leq \tau \leq-\frac{1}{2} T_{2}+T_{1}\end{cases}
$$

For this voltage profile, the barrier penetration depth $\hat{T}_{1}$ for a particle with an energy deviation $\Delta \hat{E}$ is given by

$$
\hat{T}_{1}=\frac{1}{2} \frac{|\eta| T_{\text {rev }}}{\beta^{2} E_{0} e V_{0}}(\Delta \hat{E})^{2}
$$

The barrier bucket height is also given by

$$
\Delta E_{\text {bucket }}=\left[2 \frac{\beta^{2} E_{0}}{|\eta| T_{\mathrm{rev}}} e V_{0} T_{1}\right]^{\frac{1}{2}}
$$

and the synchrotron period of oscillations in the barrier bucket is given by

$$
T_{\mathrm{s}}=2 \frac{\beta^{2} E_{0}}{|\eta|} \frac{T_{2}}{\Delta \hat{E}}+4 \frac{T_{\mathrm{rev}}}{e V_{0}} \Delta \hat{E} .
$$


For JLEIC, $T_{2}$ would be the length equal to 13 buckets at $h=28$, and $T_{1}$ is equal to the length of half a bucket. The magnitudes are shown in Table 13.

TABLE 13: JLEIC Barrier Bucket Parameters.

\begin{tabular}{llcc}
\hline \hline Length & units & $T_{1}$ & $T_{2}$ \\
\hline time & $\mu \mathrm{s}$ & 0.128 & 3.336 \\
Buckets $(h=28)$ & - & 0.5 & 13 \\
Distance & $\mathrm{m}$ & 79.64 & 1035.36 \\
\hline \hline
\end{tabular}

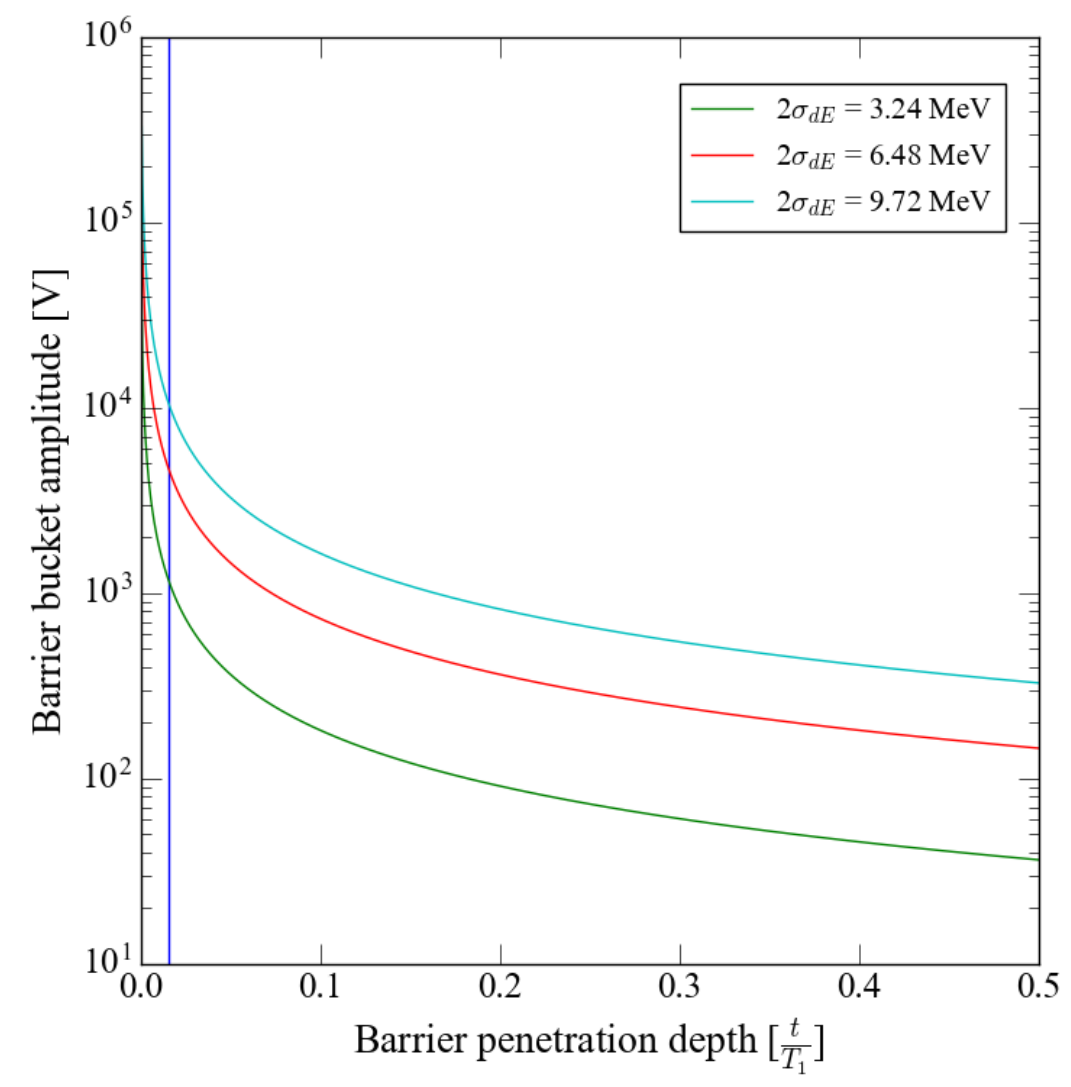

FIG. 33: Barrier bucket voltage amplitude vs the penetration depth (normalized to T1). For a complete adiabatic debunch the $95 \%$ energy spread is $2 \sigma_{d E}=3.48 \mathrm{MeV}$. Blue line is at the length of one RF bucket of $h=3584$.

Leaving a larger penetration depth would result in a longer coasting beam which in turn 
produces additional filled buckets when the RF system at $h=3584$ ramps up to capture the beam. Fig. 33 shows the required barrier bucket voltage $v s$ the barrier penetration depth. A line at the depth equal to one bucket length at $h=3584$ is indicated as the tolerance. At this level, a perfectly adiabatic debunched beam requires a barrier bucket voltage of $1 \mathrm{kV}$.

The barrier buckets in the Fermilab recycler ring operated at $2 \mathrm{kV}$ [34], while the barrier bucket system currently being developed for the SIS 100 synchrotron at GSI [35] is set to operate at $1 \mathrm{kV}$. Moderately higher voltage amplitude barrier buckets have been successfully tested. A $40 \mathrm{kV}$ ferrite loaded RF cavity developed by BNL and a magnetic alloy loaded cavity developed by KEK have been successfully tested at AGS [36]. Thus, the barrier bucket voltages required by JLEIC are within the current existing limits.

The main concern for the barrier bucket beam is having small Landau damping; this can lead to higher sensitivity to instabilities. The tune spread vs. energy spread of the bunch within barrier buckets for JLEIC parameters is shown in Fig. 34 for several barrier voltages.

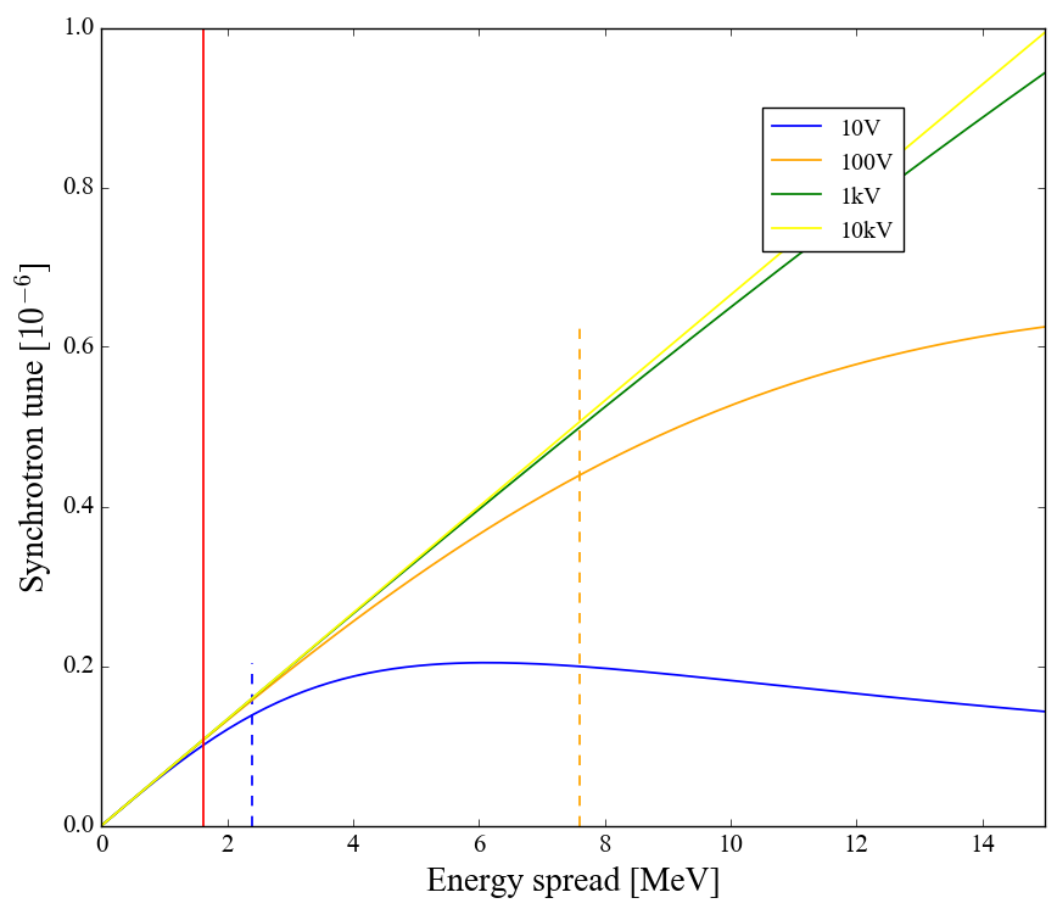

FIG. 34: Synchrotron tune plotted against the energy offset of the bunch for different barrier bucket voltages. The dashed lines shows the bucket acceptance for each voltage. $1 \mathrm{kV}$ and $10 \mathrm{kV}$ bucket acceptance lines out of range in the plot. The vertical red line shows the $95 \%$ energy offset for the JLEIC barrier bucket beam.

With an rms energy offset of $1.62 \mathrm{MeV}$, the $95 \%$ energy offset for JLEIC parameters is $3.24 \mathrm{MeV}$. This corresponds to a tune spread in the range of $10^{-7}$. Having a beam 
with a low tune spread introduces the question of longitudinal stability because the Landau damping [37] may not be enough to overcome the instability growth.

\subsubsection{KEIL-SCHNELL STABILITY CRITERION}

In this section the theory behind the Keil-Schnell stability criterion for coasting beams is developed. Let $\tau$ be the time difference between a test particle and the design particle crossing the same point of the machine in one revolution. Then the equation of motion can be written as

$$
\ddot{\tau}=\frac{\eta}{p_{0} / e}[E+v \times B] .
$$

A distribution function with a small perturbation that has a oscillation frequency of $\omega_{\text {nc }}$ can be written as

$$
\Psi(\tau, \dot{\tau}, t)=g(\dot{\tau})+g_{n}(\dot{\tau}) e^{i\left(n \omega_{0} \tau+\omega_{\mathrm{nc}} t\right)}
$$

The A.C part of the perturbation is

$$
S_{n}(t, \theta)=\frac{2 \pi I}{\omega_{0}} \int_{\dot{\tau}} g_{n}(\dot{\tau}) d \dot{\tau} e^{i\left[\left(n \omega_{0}+\omega_{\mathrm{nc}}\right) t-n \theta\right.}
$$

The impedance creates an electromagnetic field on the beam axis which is

$$
[E+v \times B]=-\frac{I}{\beta_{0} c} Z\left(n \omega_{0}+\omega_{\mathrm{nc}}\right) \int_{\dot{\tau}} g_{n}(\dot{\tau}) d \dot{\tau} e^{i\left[\left(n \omega_{0}+\omega_{\mathrm{nc}}\right) t-n \theta\right.} .
$$

Using Liouville's theorem it can be deduced that the distribution function over time should remain constant, which leads to $d \Psi / d t=0$ expanded,

$$
\frac{\partial \Psi}{\partial t}+\frac{\partial \Psi}{\partial \tau} \dot{\tau}+\frac{\partial \Psi}{\partial \dot{\tau}} \ddot{\tau}=0
$$

known as the Vlasov's equation. Substituting for the distribution function and neglecting the higher order terms it can be reduced to

$$
g_{n}(\dot{\tau})=i \ddot{\tau} e^{i\left(n \omega_{0} \tau+\omega_{\mathrm{nc}} t\right)} \frac{\partial g_{0} / \partial \dot{\tau}}{n \omega_{0} \dot{\tau}+\omega_{\mathrm{nc}}} .
$$

Substituting for $\ddot{\tau}$, the dispersion relation for any type of particles can be written as [38]

$$
1=\frac{-\eta(q / A) I}{\left(\left(m_{0} c^{2} / e\right) \gamma \beta^{2}\right.} i \frac{Z_{z}\left(n \omega_{0}+\omega_{\mathrm{nc}}\right)}{n \omega_{0}} \int_{\dot{\tau}} \frac{\left(\partial g_{0} / \partial \dot{\tau}\right)}{\left(\omega_{\mathrm{nc}} / n \omega_{0}\right)+\dot{\tau}} d \dot{\tau}
$$

where $\eta$ is the slip factor, $q / A$ is the charge per atomic weight, $e$ is the fundamental charge, $I$ is the beam current, $m_{0} c^{2}$ is the rest mass energy, $\gamma$ and $\beta$ are relativistic parameters, $\omega_{0}$ is the revolution angular frequency, $Z_{z}$ is the longitudinal impedance, $\omega_{n c}$ is the oscillating 
frequency of the perturbation, and $\tau$ is the time delay between the design and the test particle.

For a coasting beam with a parabolic distribution for the momentum spread,

$$
g_{0}(\dot{\tau})=\frac{3 \omega_{0}}{8 \pi \dot{\tau}_{L}}\left(1-\frac{\dot{\tau}^{2}}{\dot{\tau}_{L}^{2}}\right)
$$

With $|\dot{\tau}|<\dot{\tau}_{L}$ and $\int_{\dot{\tau}} g_{0}(\dot{\tau}) d \dot{\tau}=\omega_{0} / 2 \pi$,

$$
\frac{\partial g_{0}}{\partial \dot{\tau}}=-\frac{3 \omega_{0}}{4 \pi \dot{\tau}_{L}^{3}} \dot{\tau}
$$

Then the integral part of the dispersion relation can be found for this distribution as

$$
\int_{-\infty}^{+\infty} \frac{\left(\partial g_{0} / \partial \dot{\tau}\right)}{\left(\omega_{\mathrm{nc}} / n \omega_{0}\right)+\dot{\tau}} d \dot{\tau}=-\frac{3 \omega_{0}}{4 \pi \dot{\tau}_{L}^{3}} \int_{-\tau_{L}}^{\tau_{L}} \frac{\dot{\tau}}{\dot{\tau}+\left(\omega_{\mathrm{nc}} / n \omega_{0}\right)} d \dot{\tau}
$$

Using the substitution $x=\dot{\tau} / \tau_{L}$ and $d \dot{\tau}=\tau_{L} d x$

$$
\int_{-\infty}^{+\infty} \frac{\left(\partial g_{0} / \partial \dot{\tau}\right)}{\left(\omega_{\mathrm{nc}} / n \omega_{0}\right)+\dot{\tau}} d \dot{\tau}=-\frac{3 \omega_{0}}{4 \pi \dot{\tau}_{L}^{2}} \int_{-1}^{+1} \frac{x}{x+\left(\omega_{\mathrm{nc}} / n \omega_{0}\right) \dot{\tau}_{L}} d \dot{\tau}=-\frac{3 \omega_{0}}{4 \pi \dot{\tau}_{L}^{2}} J_{z}
$$

With $\dot{\tau}=\eta_{c} \delta$ and $\dot{\tau}_{L}=\eta_{c} \delta_{L}=\left(\eta_{c} / \sqrt{2}\right) \delta_{F W H M}$ for parabolic distribution, the dispersion relation becomes

$$
J_{z}^{-1}=i\left(\frac{Z_{z}\left(n \omega_{0}\right)}{n}\right)\left(\frac{3 I_{0}}{2 \pi \eta_{c}\left(m_{0} c^{2} / q\right) \beta^{2} \gamma \delta_{F W H M}^{2}}\right)
$$

by setting

$$
\Lambda_{z}=\frac{3 I_{0}}{2 \pi \eta_{c}\left(\frac{m_{0} c^{2}}{q}\right) \beta^{2} \gamma \delta_{F W H M}^{2}} .
$$

Then the real and imaginary parts of $J_{z}^{-1}$ can be written as

$$
\begin{array}{r}
\operatorname{Im}\left(J_{z}^{-1}\right)=\Lambda_{z} \operatorname{Re}\left(\frac{Z_{z}\left(n \omega_{0}\right)}{n}\right) \\
\operatorname{Re}\left(J_{z}^{-1}\right)=-\Lambda_{z} \operatorname{Im}\left(\frac{Z_{z}\left(n \omega_{0}\right)}{n}\right) .
\end{array}
$$

A stability diagram can be then achieved by plotting the Imaginary and the real parts of $J_{z}^{-1}$ 


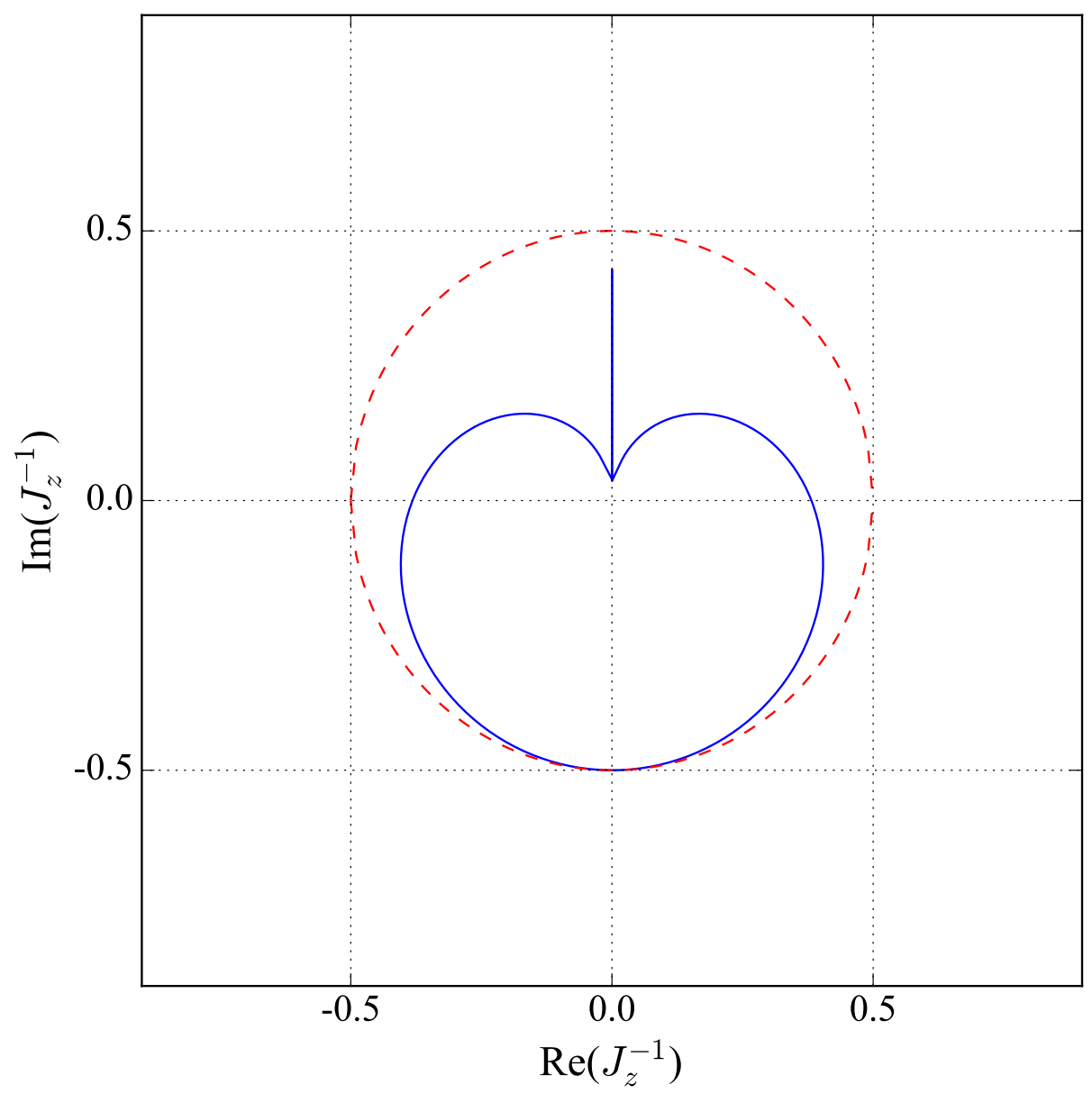

FIG. 35: Stability diagram.

The stable region is within the region of the circle. For a coasting beam with rounded edges this corresponds to $\Lambda_{z}|Z / n| \leq 0.5$ and results in the stability criterion for broad band impedance model (derived in a paper published in 1969 by E. Keil and W. Schnell [39]),

$$
\left|\frac{Z_{z}\left(n \omega_{0}\right)}{n}\right| \leq \frac{\left(m_{0} c^{2} / q\right) \eta \beta^{2} \gamma}{I_{0}} \delta^{2} .
$$

A good approximation for the bunched beams can be achieved by replacing the average current $\left(I_{0}\right)$ by the peak current $(\hat{I})$ in the Keil-Schnell criterion

$$
\begin{gathered}
I_{0} \Rightarrow \hat{I}=\frac{e c N_{0}}{\sqrt{2 \pi} \sigma_{z}} \\
\left|\frac{Z_{z}\left(n \omega_{0}\right)}{n}\right| \leq \sqrt{\frac{\pi}{2}} \frac{Z_{0}}{r_{p}} \frac{|\eta| \gamma}{\beta} \frac{\sigma_{z} \sigma_{\delta}^{2}}{N_{b}}
\end{gathered}
$$

which is known as the Keil-Schnell-Boussard criterion [39] [40]. 


\subsection{METHOD}

Due to the low emittance growth allowed for JLEIC, iso-adiabatic ramps are considered for all the voltage ramps for the Debunch/Rebunch method. After accelerating the 26 bunches at $h=28$, the RF voltage $\left(V_{\mathrm{i}, \mathrm{deb}}\right)$ is ramped down to $V_{\mathrm{f}, \mathrm{deb}}$ before stepping down to zero, allowing the beam to drift long enough to get a fully coasting beam. Following, the RF voltage $V_{\mathrm{i}, \mathrm{reb}}$ is turned on and ramped up to the final voltage $V_{\mathrm{f} \text {,reb. Using iso-adiabatic }}$ curves increases the ramping time while reducing the emittance growth. The RF voltage change for the debunch/rebunch process is shown in Fig. 36

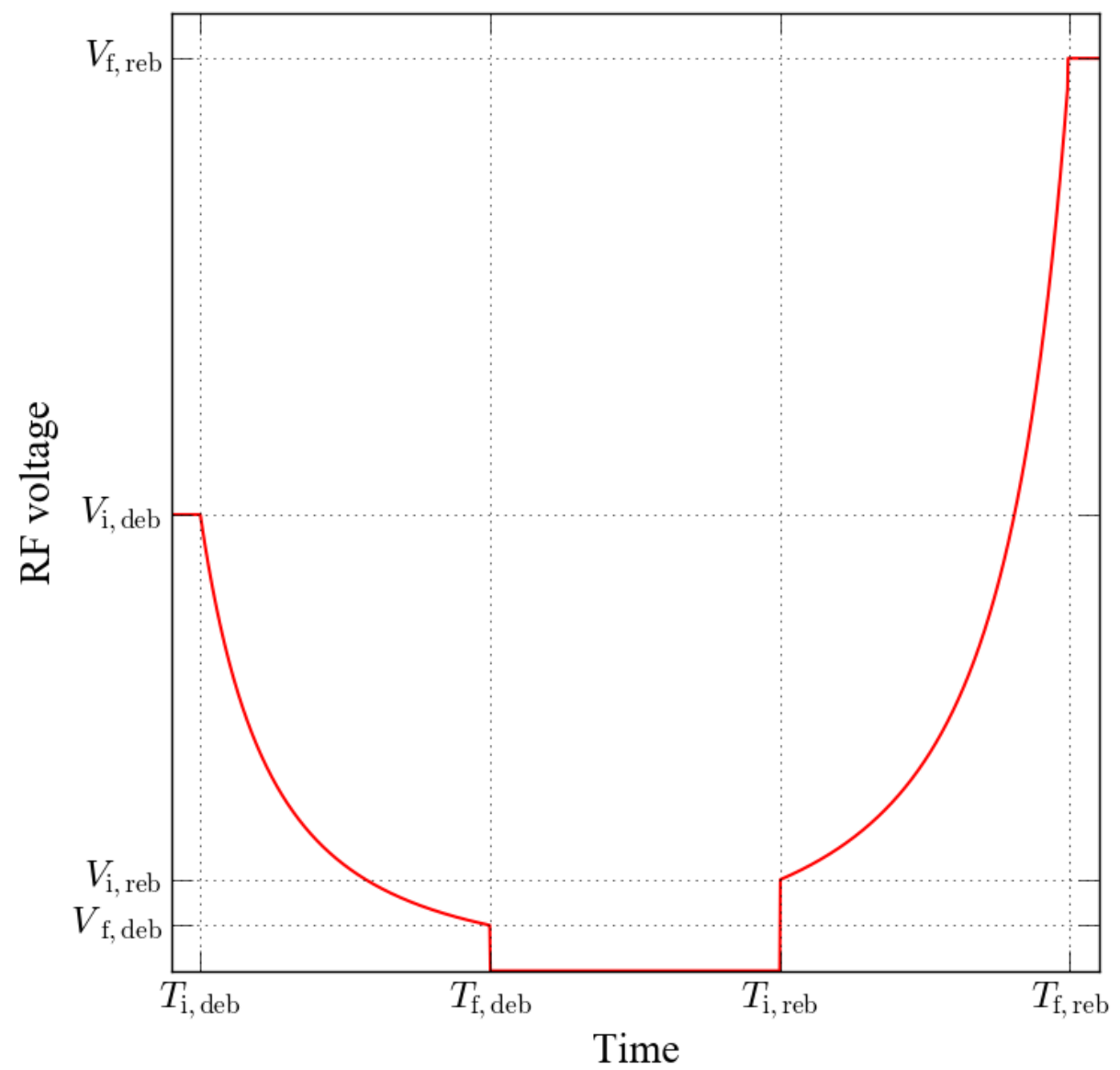

FIG. 36: RF voltage ramps for a debunch rebunch method.

An approximation for the time [41] needed for debunching is given by

$$
T_{\text {debunch }}=\frac{\pi-\Delta \phi}{h \omega_{R}|\eta| \frac{\Delta p}{p}} .
$$

For JLEIC at $h=28$ this is about 0.15 seconds. However, this time is only for the head of the bunch to reach the tail of the adjacent bunch. In reality more time is needed for the 
debunched beam to be transformed to a fully coasting beam that is uniform on the time coordinate. For the RF voltage ramps, a good approximation [13] is given by

$$
t_{\mathrm{ramp}}=\frac{1}{\omega_{s}\left(V_{d}\right) \epsilon}
$$

where $V_{d}$ is the lowest voltage the ramp will go to before jumping down to zero. For the case of rebunching this will be the starting RF voltage amplitude. $\epsilon$ is the adiabatic parameter, usually preferred to be $<0.1$. As seen in Fig. 8 in [41] the emittance growth is dependent on the ratio of bunch emittance to bucket area $\left(\epsilon_{r}\right)$. In this case the bucket area is taken from the smallest non-zero RF voltage of the ramping process. For the debunch ramp shown in Fig. 36 this would be the bucket area at $V=V_{\mathrm{f}, \mathrm{deb}}$. For the JLEIC bunch size, an emittance ratio of one is obtained when the final voltage $V_{d}$ is in the range $100 \mathrm{~V}$.

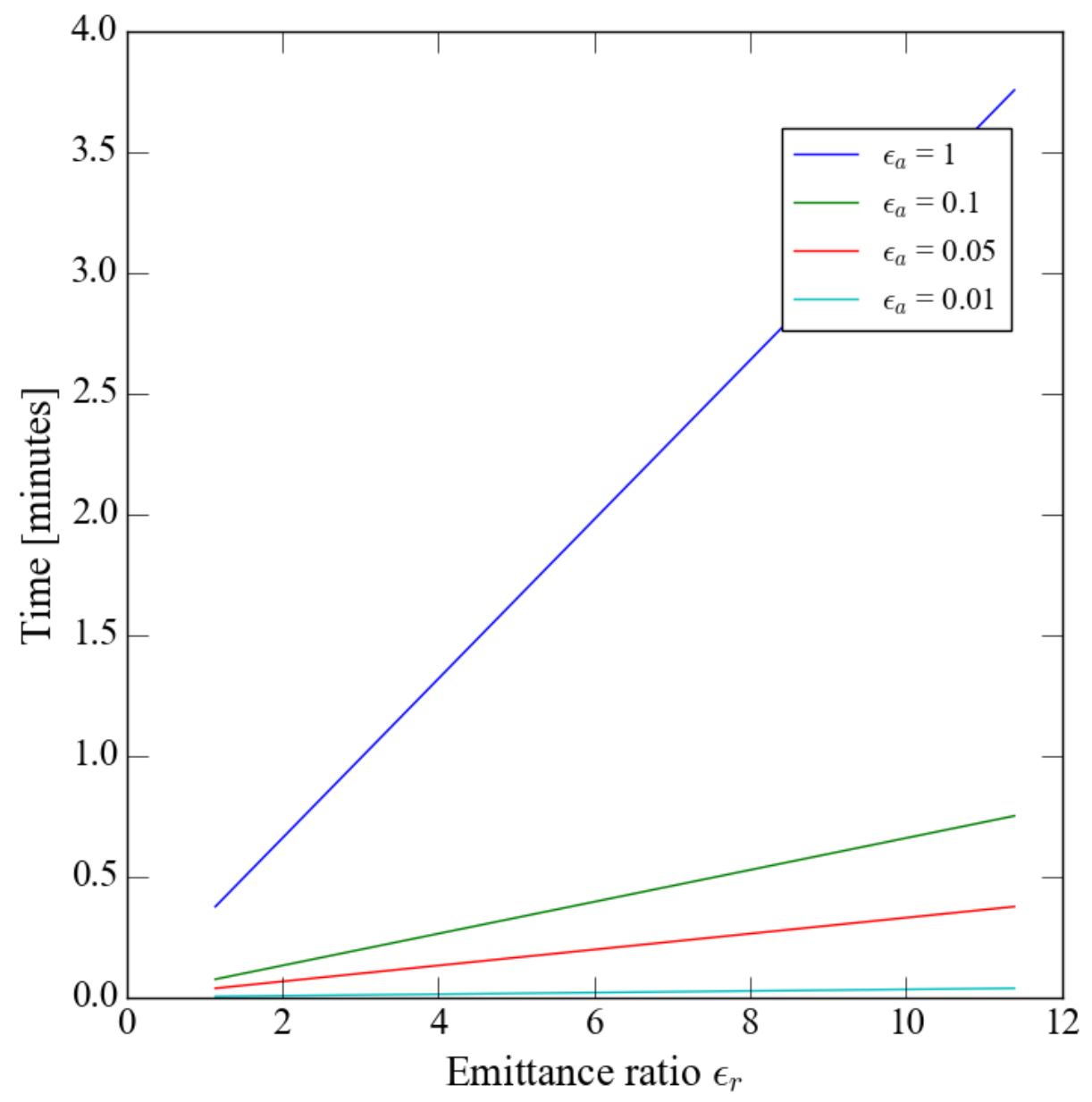

FIG. 37: Required time for an iso adiabatic voltage ramp plotted against the final RF amplitude for different adiabaticity parameters. 
After injecting 26 bunches from the ion booster, they are kept in $h=28$ harmonic RF. The barrier pulses are then created in the places of empty buckets as seen in Fig. 38

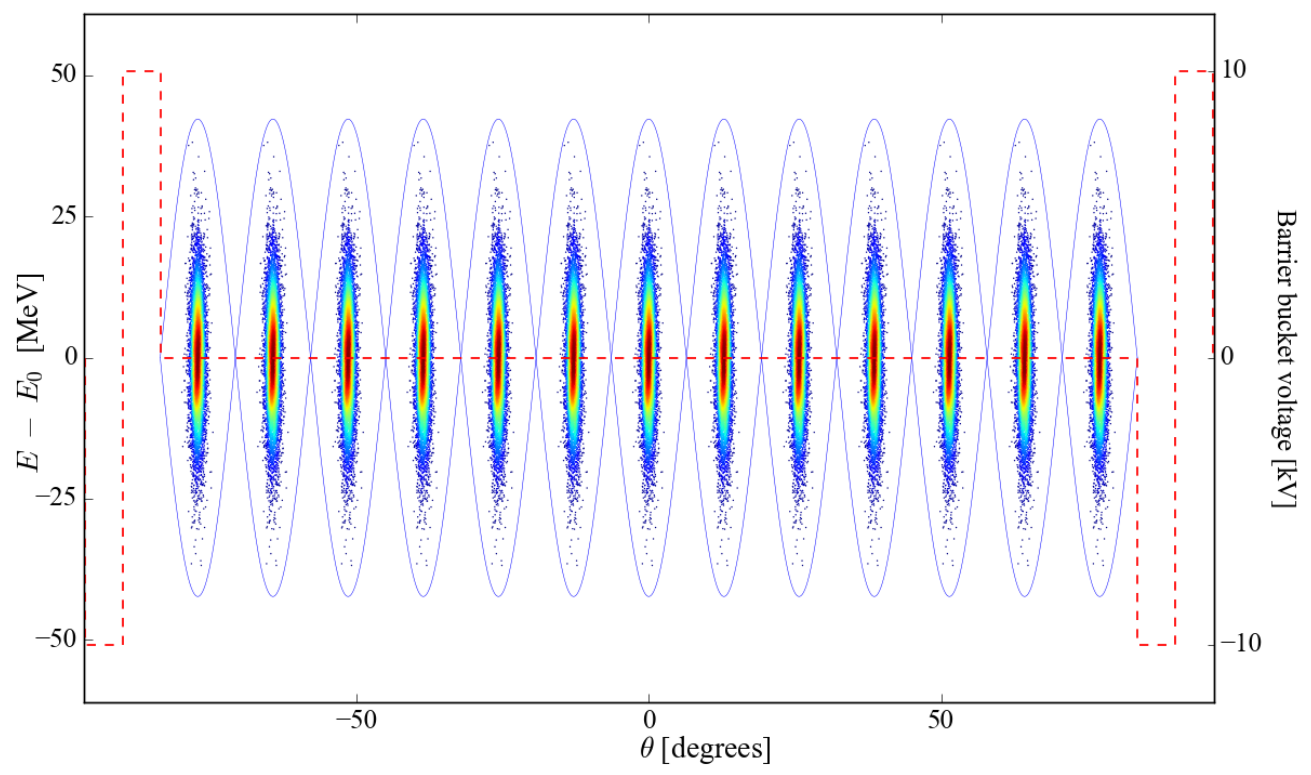

FIG. 38: 13 bunches inside two barrier buckets.

The JLEIC ion collider ring has two such formations with two barrier waveforms separating them in to two groups. Debunching the beam with iso-adiabatic ramps results in a coasting beam between each barrier pulse.

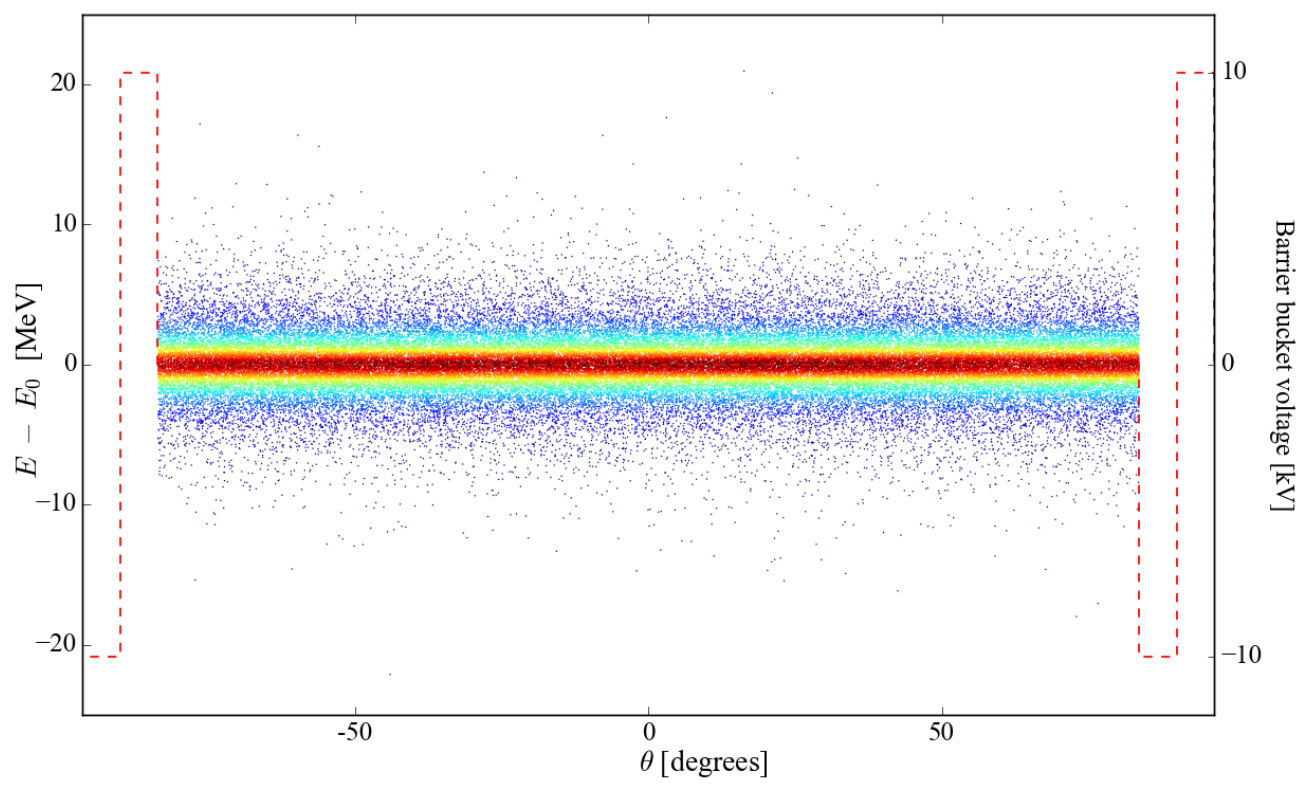

FIG. 39: Adibatically debunched beam from 13 bi Gaussian bunches. 
In a perfectly adiabatic debunching process (given a long enough time) the debunched beam would preserve the longitudinal emittance. The resulting beam of such a process has an energy spread of $d E / E=1.62 \times 10^{-5}$. But as seen in Fig. 39 the resulting debunched beam shows significant emittance growth. This is with an adiabaticity parameter value 0.1. For debunching the beam with different values for the parameter, Fig. 40 shows the emittance growth.

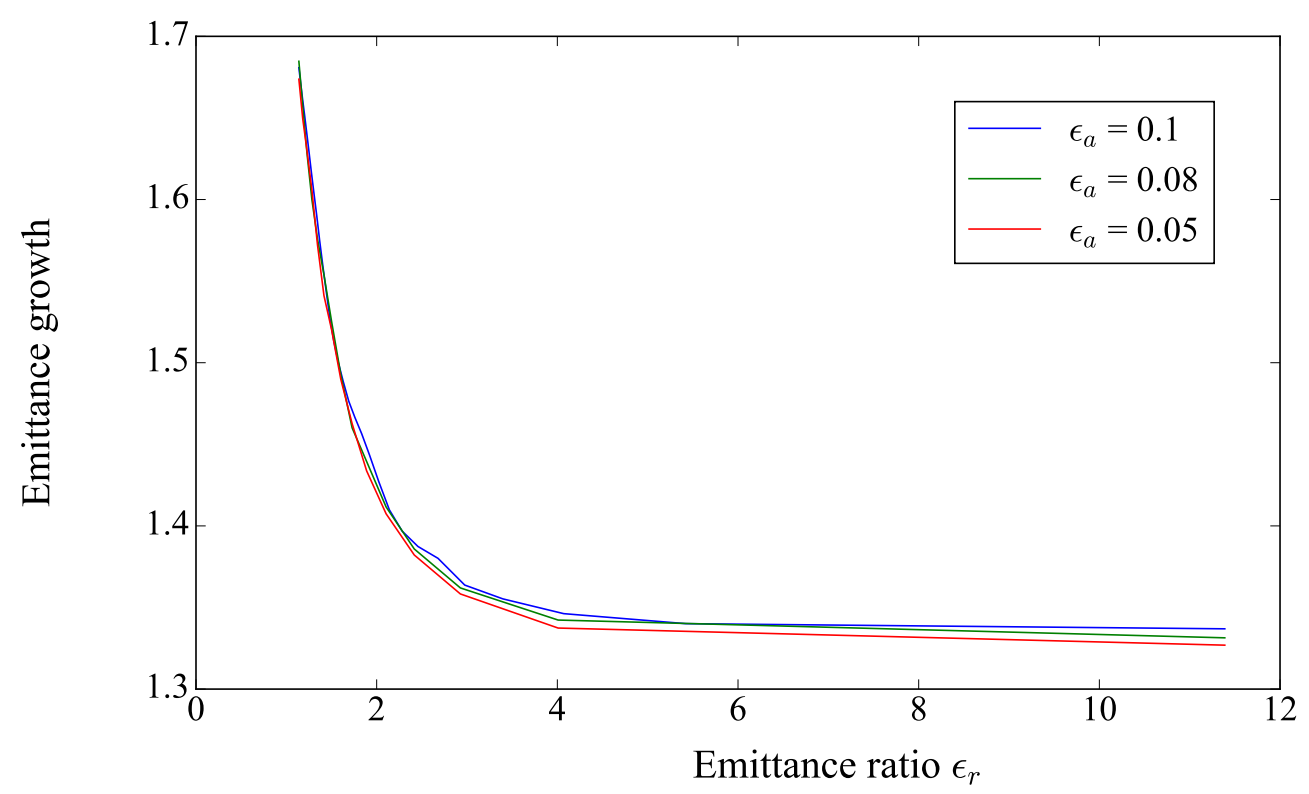

FIG. 40: Emittance growth (final emittance to initial emittance ratio) for iso-adiabatic debunching.

This indicates that lowering the adiabaticity parameter will help to keep the emittance growth lower but it will increase the ramping time to several minutes.

\subsection{BROADBAND IMPEDANCE THRESHOLDS}

A threshold for the longitudinal impedance for the beam in a barrier bucket can be found using the Keil-Schnell Criterion (Eq. 99)

$$
\left|\frac{Z}{n}\right| \simeq 0.99 \Omega .
$$

The threshold for microwave instability for the Fermilab recycler barrier bucket beams were calculated using the Keil-Schnell-Boussard criterion (Eq. 101). For JLEIC the bunches can be treated as coasting beams rather than bunched beams due to being considerably longer 
(almost half the circumference of the ring), so the threshold was calculated using Eq. 99

$$
\left|\frac{Z}{n}\right| \simeq 1.16 \Omega
$$

These estimates are calculated using the assumption that the debunching is purely adiabatic, but in reality an emittance growth can be observed during the debunching. The emittance growth for a coasting beam is directly proportional to the momentum spread of the beam. From the JLEIC 2018 spring collaboration meeting [42], the most recent estimates for the longitudinal impedance budget was $0.5 \Omega$. This is in the same order of magnitude as the thresholds found by the Keil-Schnell Criterion, with the emittance growth still being well over the allowed tolerance of $10 \%$ for adiabaticity parameter values ranging 0.1 to 0.05 .

\subsection{NARROW BAND IMPEDANCE}

There are also effects from the presence of narrow band impedance in the ring. The cavity used for the simulation was a five cell SRF cavity operating at $952.6 \mathrm{MHz}$. This is used to shorten the bunches after the rebunching process, resulting in every other bucket being filled except for the area within the abort gaps. Undamped HOM's were used in the simulation for $952.6 \mathrm{MHz}$ cavity preliminary design [43]. Fig. 41 shows the monopole impedance vs. frequency curve for this cavity.

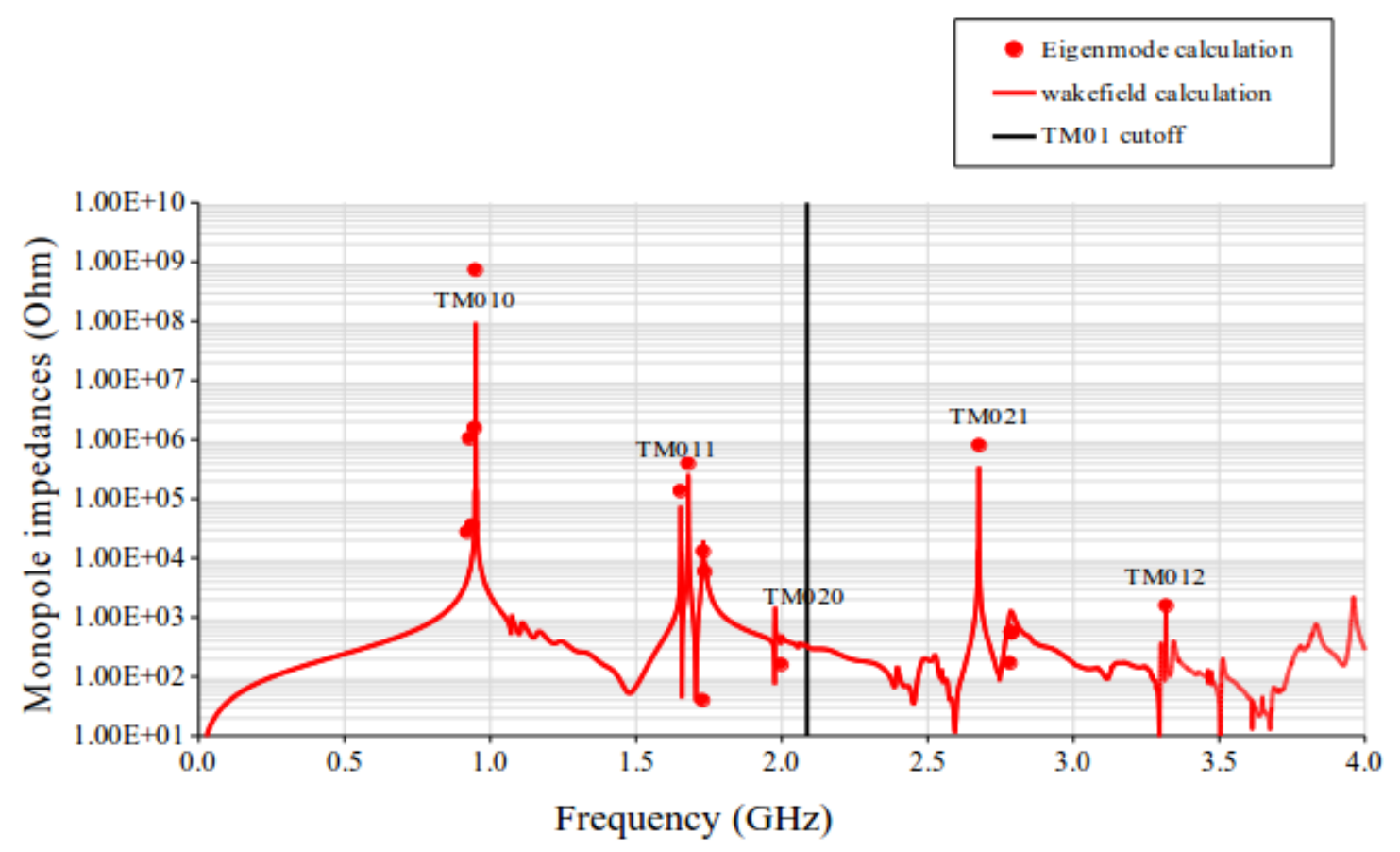

FIG. 41: Longitudinal impedance vs frequency of the 5-cell $952.6 \mathrm{MHz}$ cavity. 
All the modes that had a value above $R / Q=10$ were selected for the simulation since higher $R / Q$ has a higher effect on the instability of the beam.

TABLE 14: modes of the 5-cell cavity selected for the simulation.

\begin{tabular}{lcc}
\hline \hline Frequency $[\mathrm{MHz}]$ & Mode & $\mathrm{R} / \mathrm{Q}$ \\
\hline 1652.616 & TM011 & 16.26 \\
1679.291 & TM011 & 63.61 \\
1731.045 & TM011 & 37.71 \\
1735.417 & TM011 & 31.64 \\
2676.960 & TM021 & 16.53 \\
\hline \hline
\end{tabular}

A simulation of a coasting beam between two barrier pulses was done using the modes given in Table 14 produced the results in Fig. 42.

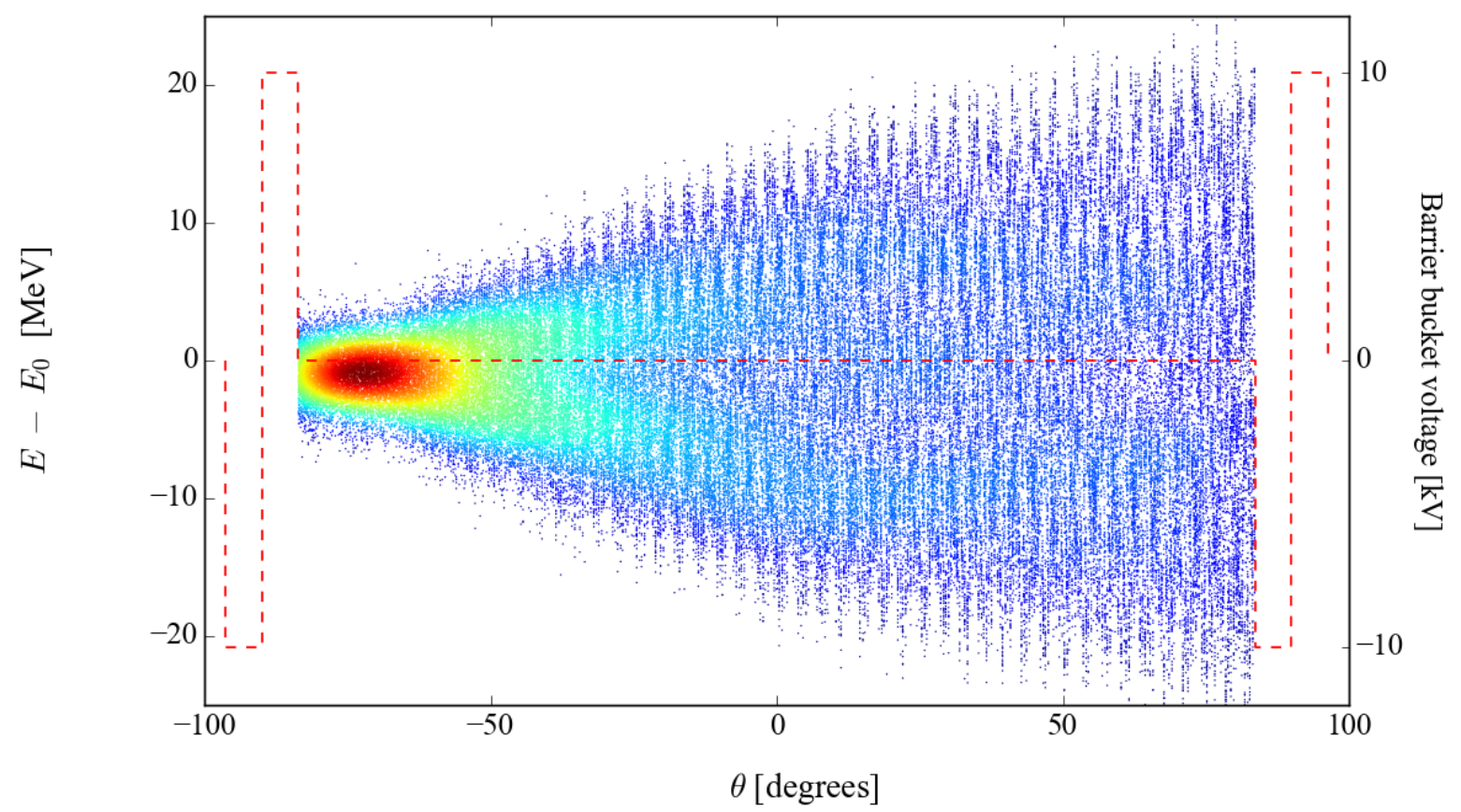

FIG. 42: Beam profile at $T=1 \mathrm{~ms}$, for coasting beam instability triggered by a single JLEIC 952.6 MHz SRF cavity narrow band impedance.

The beam energy spread grew considerably as soon as the simulation began. Within 10 
milliseconds the rms energy spread went up by a factor of 10. The rms energy spread over time is shown in Fig. 43 .

Coasting beam in JLEIC collider ring at $100 \mathrm{GeV}$
ERMS VS TIME

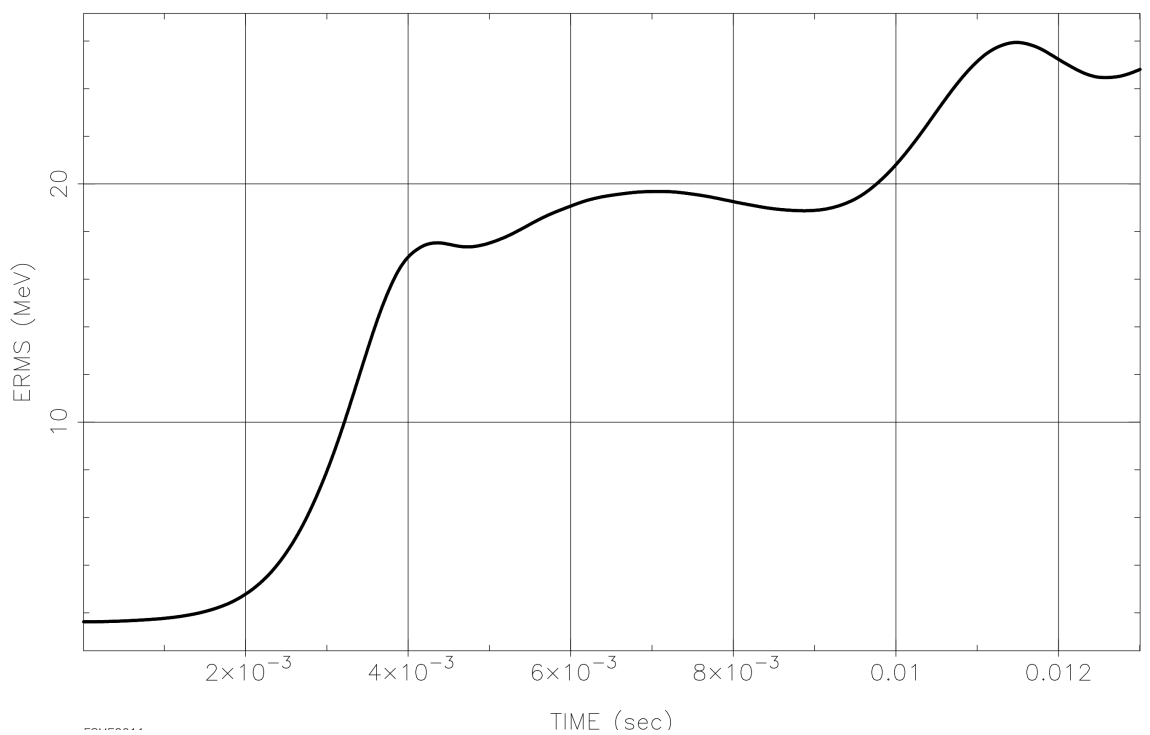

FIG. 43: Energy spread vs time, for coasting beam instability triggered by a single JLEIC 952.6 MHz SRF cavity narrow band impedance.

For the debunch/rebunch scheme to work there need to be at least two cavities in the JLEIC ion collider ring. The $952.6 \mathrm{MHz}$ cavity will be used at the final stage of the longitudinal dynamics. With this cavity having modes with high $R / Q$ the coasting beam becomes unstable within a very short period of time (less than a millisecond). The rate at which the beam was blowing up is so short that performing other longitudinal gymnastics and re-capturing them into a large number of buckets (with equal emittance values) would be impossible.

It is important to note that the simulation ran using the modes from a single 5-cell cavity. In the real collider ring there are more cavities with monopole modes having higher $R / Q$ that would in turn effect the beam. With these results it is clear that the debunching/rebunching method is not a feasible scheme to get the final number of proton bunches required for the electron ion collision at JLEIC. The beam instability growth is too large for the beam to be saved by any other RF gymnastics that we can do in a relatively short period of time. 


\section{CHAPTER 6}

\section{ADIABATIC BUNCH SPLITTING}

\subsection{BACKGROUND}

This chapter discusses another method under consideration for the JLEIC ion collider ring final bunch formation in the longitudinal dimension, namely bunch splitting. This method has been demonstrated and used for operation in CERN PS [44] for increasing the number of bunches in the ring. Unlike the debunch/rebunch method where the beam drifts in space with no control by the RF systems, bunches are kept controlled throughout the whole splitting process. Currently there are two techniques being used in the PS to split bunches for the required number of final bunches.

The current baseline bunch formation scheme for JLEIC hadron beams was described by J. Guo at the Fall 2016 JLEIC collaboration meeting [45]. This scheme accelerates the hadron beams in an $h=28 \mathrm{RF}$ system with two unfilled buckets for abort gaps. This bunch formation requires seven consecutive 1:2 harmonic bunch splits which would produce 128 equal emittance bunches, resulting in a final configuration of $h=28 \times 2^{7}=3584$, of which $26 \times 2^{7}=3328$ buckets contains the beam and $2 \times 2^{7}=256$ are empty. Assuming that there is no beam loss in the adiabatic splitting process, and final bunches will have $10^{10}$ protons/bunch per Fig. 6 , each of the $h=28$ bunches will have a bunch intensity of $1.28 \times 10^{12}$ protons.

\subsection{BUNCH MERGING AND BUNCH SPLITTING}

Bunch merging is the process where two bunches are combined into one so that the final bunch has a higher intensity, which in turn produces a high peak current as required by the collider to increase the luminosity. The method was initially proposed in 1983 [46] and was applied to the generation of the anti-proton production beam in the CERN PS [47]. This was also used to create the bunch at the output of AGS and for the nominal filling scheme to concentrate all of the ions of the booster pulse into a single bunch [48]. This process requires two RF systems with harmonics related by $h_{1}=\frac{1}{2} h_{2}$, where $h_{1}$ is the harmonic number of the first RF cavity and $h_{2}$ is the harmonic number of the second cavity. The RF voltage of 
the initial harmonic system is ramped down while the second harmonic system is ramped up to it's final voltage.

Lioville's theorem is applicable to this process since bunch splitting is the reversal of the bunch merging method. By reversing the RF ramps, a single bunch can be split in two bunches. The bunch splitting method was proposed for the PS complex after the modification for the LHC during the 97-98 winter shutdown []. The splitting process was demonstrated at the PS in March 1998 and has been used since.

A disadvantage of bunch splitting over the debunch/rebunch scheme is the dependence of the initial number of bunches on the final number. The initial or the final number of bunches needs to adjusted so that they can be achieved through either one or both of the available splitting methods. Two splitting methods have been developed so far and are being used in the PS complex. The first of these methods is the one to two (1:2) splitting process, which produces two bunches from one. If done adiabatically this produces two bunches with half the emittance of the initial bunch, and this method requires two RF systems. The one to three $(1: 3)$ splitting process, as the name suggests, produce three bunches from the initial bunch as seen in Fig. 44. This requires three RF systems with more precise control over the voltage ratios and the relative phases between harmonics. This method is useful when the initial number of bunches is related to the final number by a factor of three. However, due to delicate control requirements that have to be repeated several times for the JLEIC bunch formation this process was not considered. 


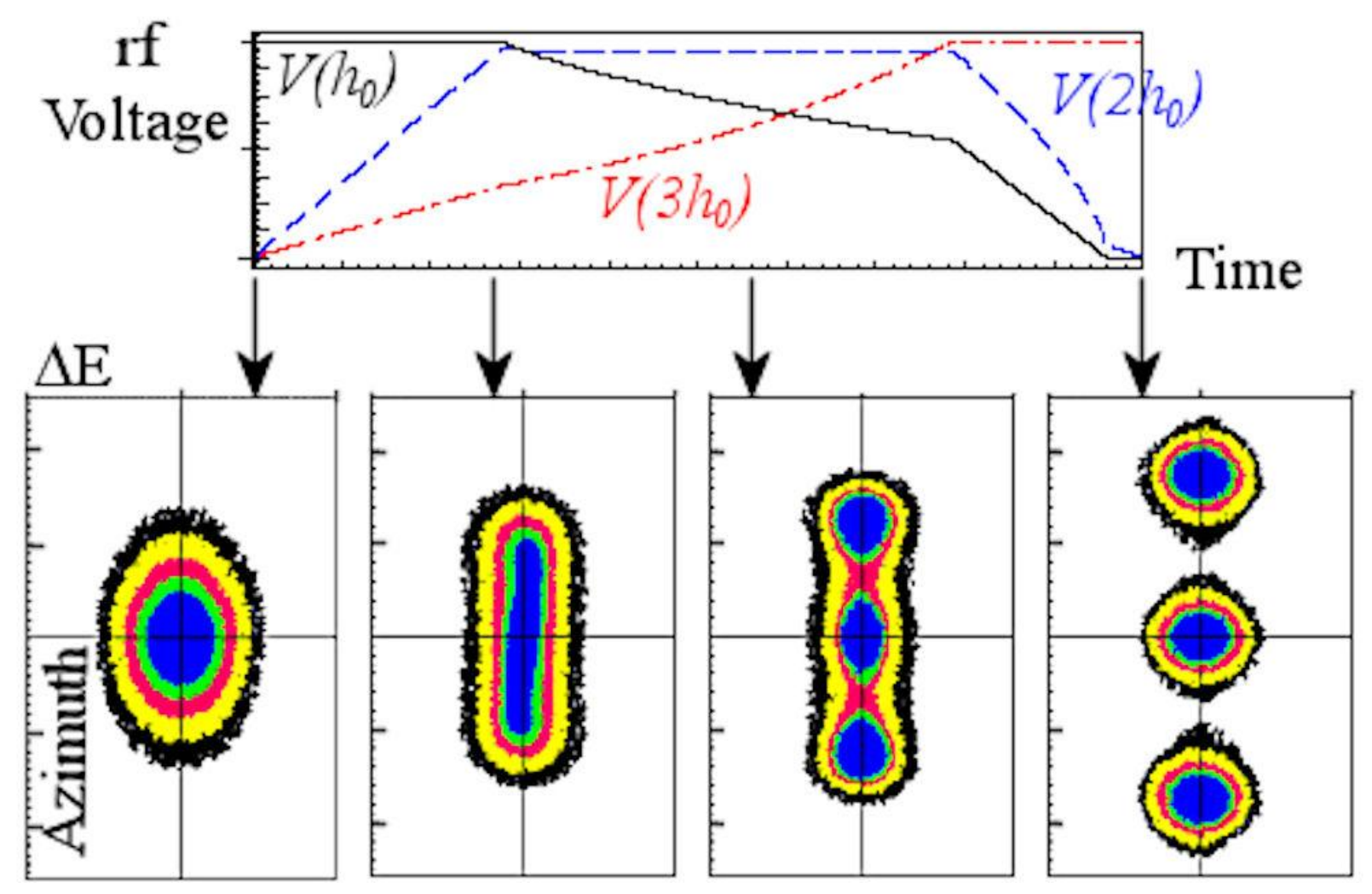

FIG. 44: 1:3 bunch splitting process in CERN PS and the corresponding RF voltage curves.

\subsection{SINGLE 1:2 SPLIT SCALING}

The RF requirements for the one to two splitting is such that the two harmonics are related by $h_{2}=2 h_{1}$ where $h_{1}$ and $h_{2}$ are the harmonic numbers of the first and the second cavities respectively. After the initial split, each consecutive split requires addition of one RF structure while preserving the relation between the initial and final harmonics and voltages. Since the area inside the RF bucket goes down with the RF frequency at a constant ratio of $V / h$, the voltages were kept at $V_{2}: V_{1}=2: 1$ for the initial iteration of a single bunch split. The two voltage ramps are linear in time with both having equal ramping times as shown in Fig. 45 


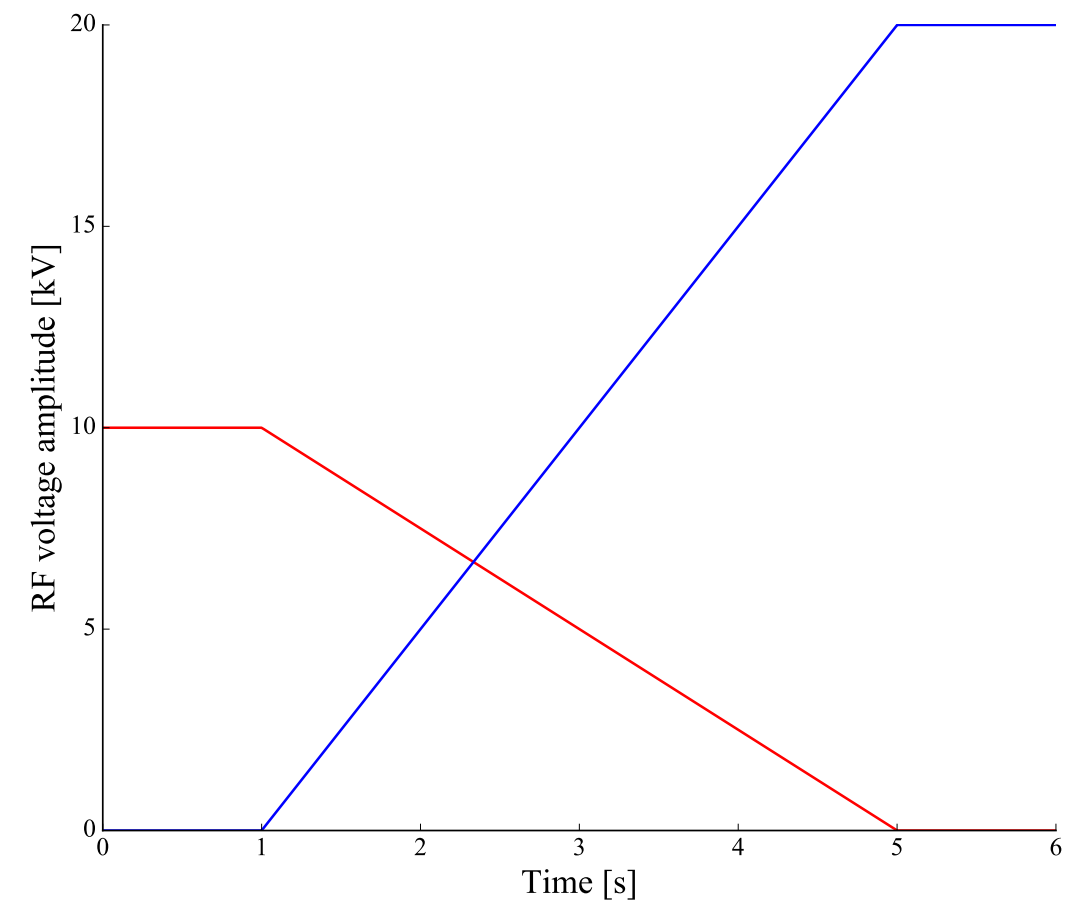

FIG. 45: RF amplitude curves for the two RF systems.

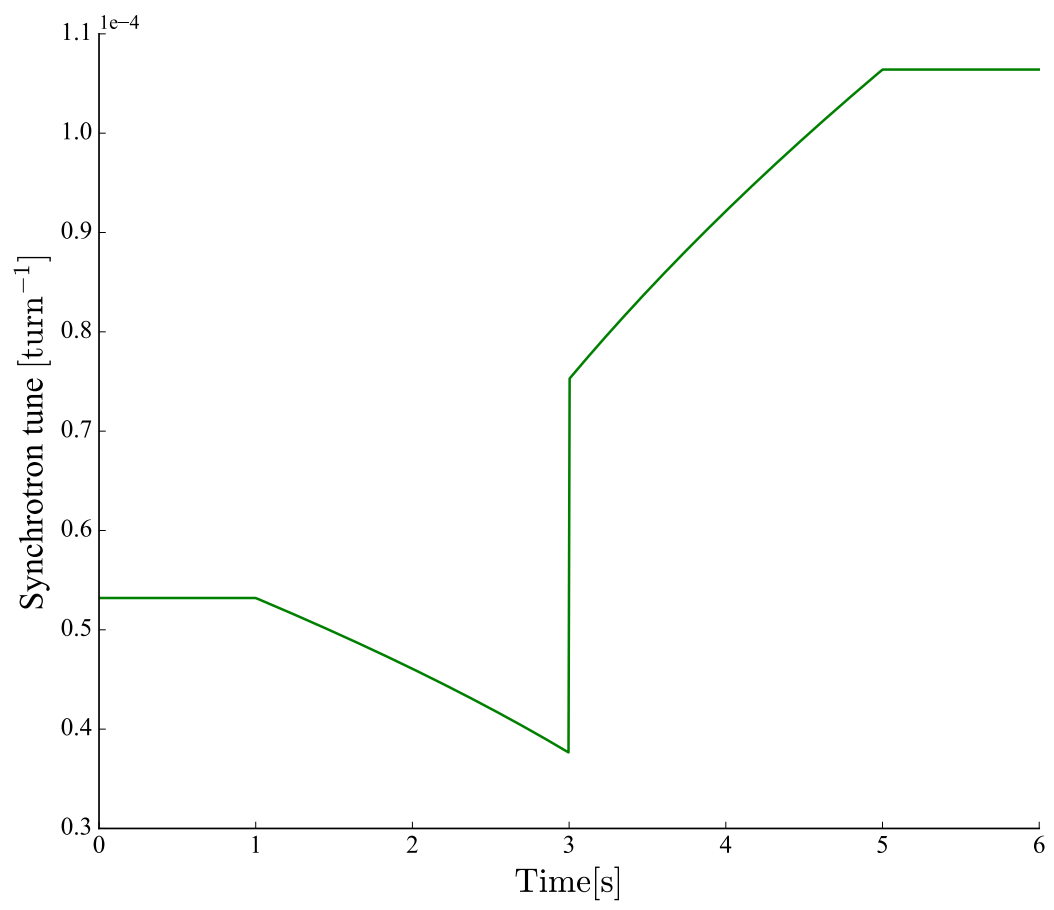

FIG. 46: synchrotron tune of the splitting process.

The time measured for the whole process is calculated in terms of synchrotron periods. 
Since there are two RF systems active during the splitting, the one with the largest synchrotron period is considered. Using this scaling makes the process repeatable for different harmonics without having to optimize for the time in seconds for each individual split. In the CERN PS it was shown that for a good split with minimum emittance blowout, the split time $T_{\text {split }}$ should be more than 5 synchrotron periods $(\mathrm{R})$. It was also mentioned that results are best when two bunches are just formed from the splitting, when the initial bunch would occupy an area in longitudinal phase space equal to $1 / 3 \mathrm{rd}$ of the bucket acceptance.

Two simulations of a single split were done using ESME with the parameters shown in Table 15

TABLE 15: JLEIC collider parameters.

\begin{tabular}{lcc} 
Parameter & Value(range) & Units \\
\hline Radius for the central orbit & 342.8 & $\mathrm{~m}$ \\
Total energy & 100 & $\mathrm{GeV}$ \\
$\gamma_{t}$ & 12.45 & - \\
$\mathrm{h}_{i}$ & 28 & - \\
$\mathrm{h}_{f}$ & 56 & - \\
$V_{R F}\left(\mathrm{~h}_{i}=28\right)$ & 10 & $\mathrm{kV}$ \\
$V_{R F}\left(\mathrm{~h}_{f}=56\right)$ & 20 & $\mathrm{kV}$ \\
Synchrotron period $\left(\mathrm{h}_{i}=28\right)$ & 178 & $\mathrm{~ms}$ \\
Synchrotron period $\left(\mathrm{h}_{i}=56\right)$ & 89 & $\mathrm{~ms}$ \\
\hline
\end{tabular}

Because RF systems double in amplitude and the harmonic number with each split, the synchrotron period, which is inversely proportional to $\sqrt{h V}$, decreases by a factor of 2 for each split. The synchrotron period propagation through the RF systems used is shown in Fig. 47 . 


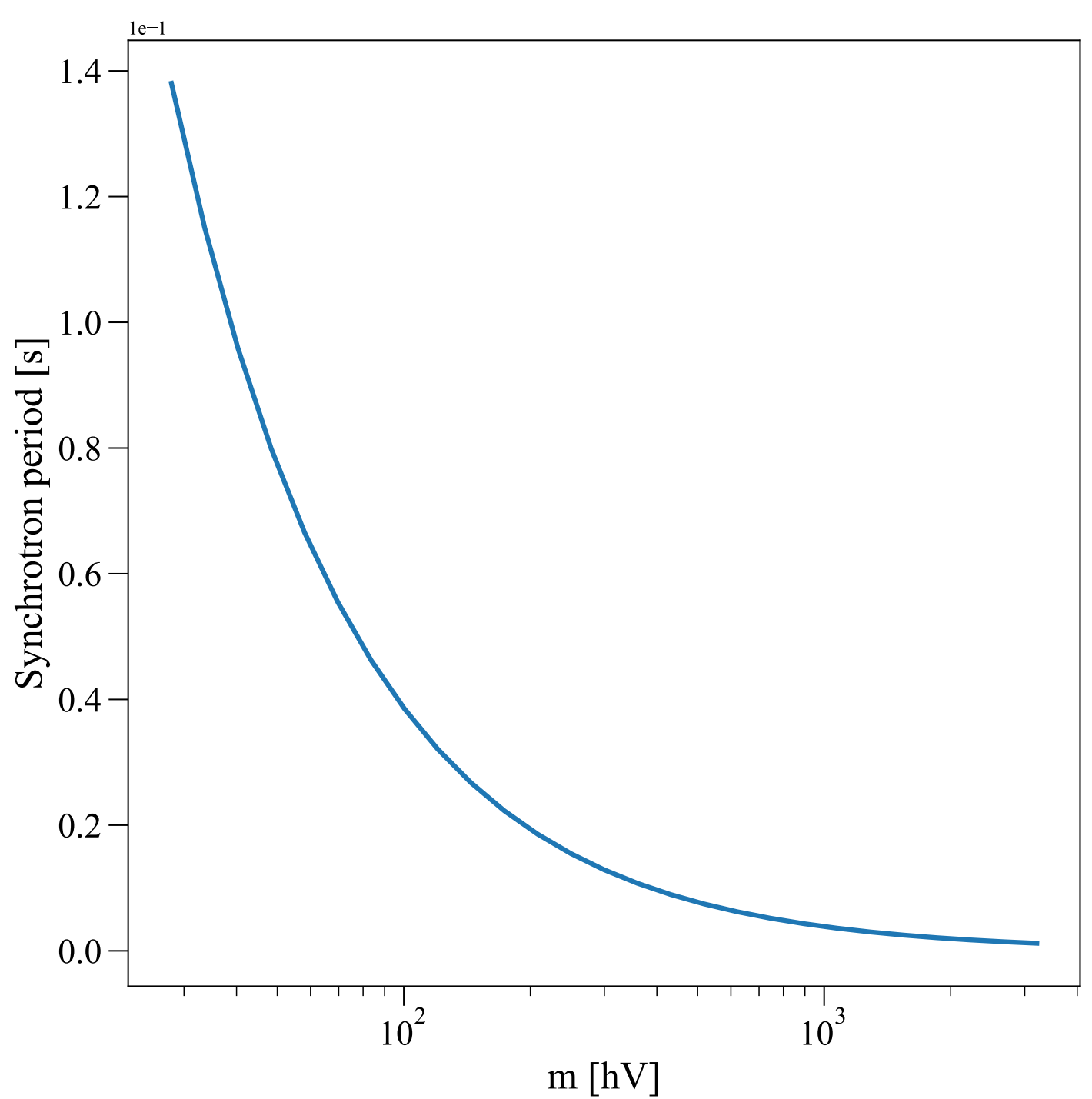

FIG. 47: Synchrotron period plotted against $m$ in log scale where $m=\frac{h V}{h_{0} V_{0}}$ with $h_{0}=28$ and $V_{0}=10 \mathrm{kV}$ for JLEIC parameters.

The bunch limiting contour, which ESME takes as the input for the emittance, is defined as the area in phase space where all the particles are inside. ESME takes this parameter as the emittance input. For a bunch at $h=28$ which has an rms emittance of $0.48 \mathrm{eV}$-s the total emittance is about $5.76 \mathrm{eV}$-s. The ESME simulation was run for 1.38 seconds which is 10 synchrotron periods for the $T_{\text {split }}$, where $T_{\text {split }}=T_{2}-T_{1}$ is taken as the time it takes to ramp the $R F$ voltages in both systems (Fig. 45). 


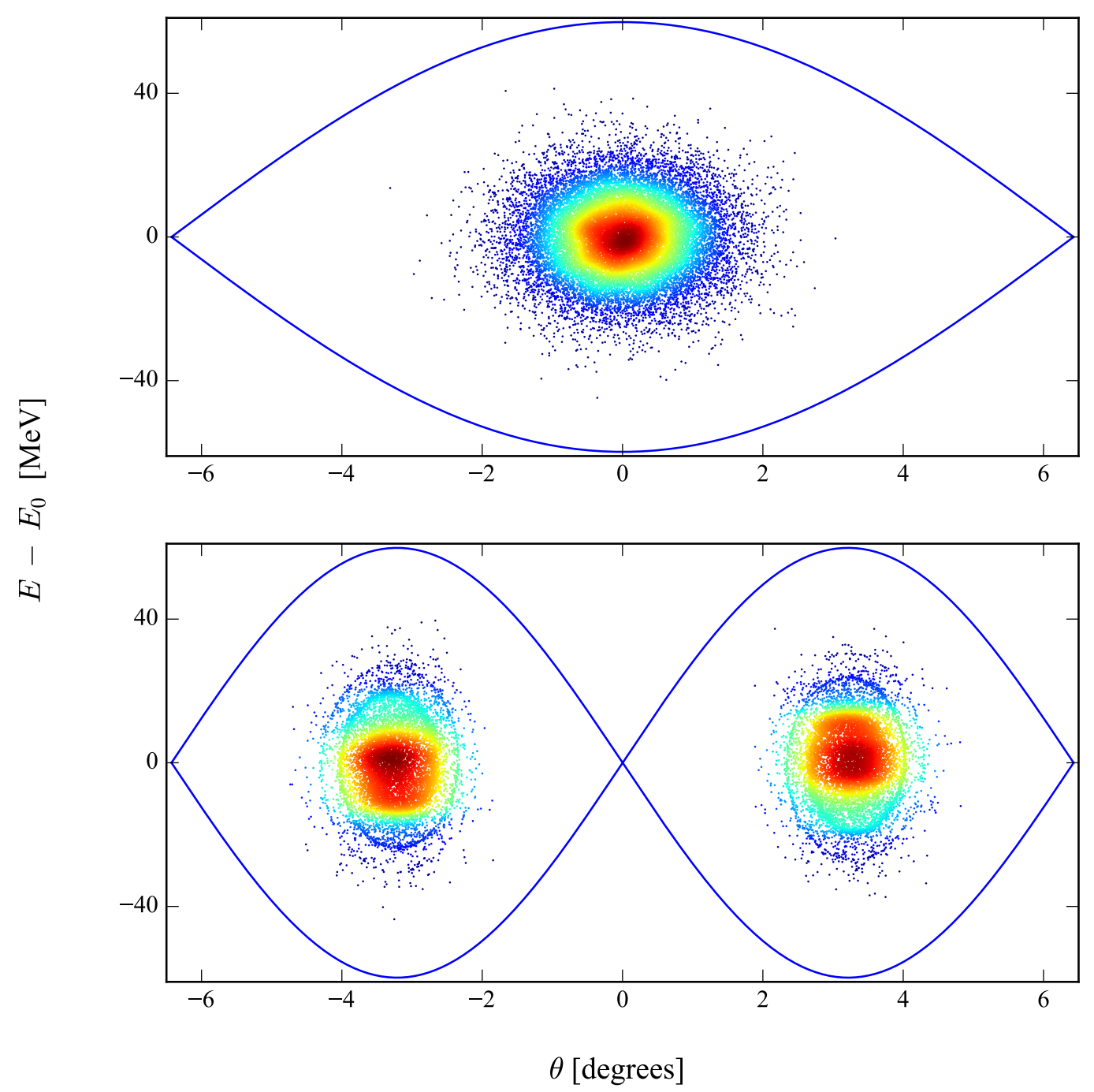

FIG. 48: Bunch splitting for $T_{\text {split }}=10 T_{\mathrm{s}}$.

The splitting process produced two bunches with equally split emittances but showed a collective emittance growth of $11 \%$. Upon closer inspection it was observed that the resulting bunches had a decoherence formed as seen in Fig. 49. This decoherence creates asymmetric bunches that in turn creates more asymmetric bunches as the splitting process continues. The decoherence can be minimized by letting the bunch settle in the bucket before starting the next split. But the time it requires for the decoherence to minimize to an acceptable amount is still large compared to the split time. 


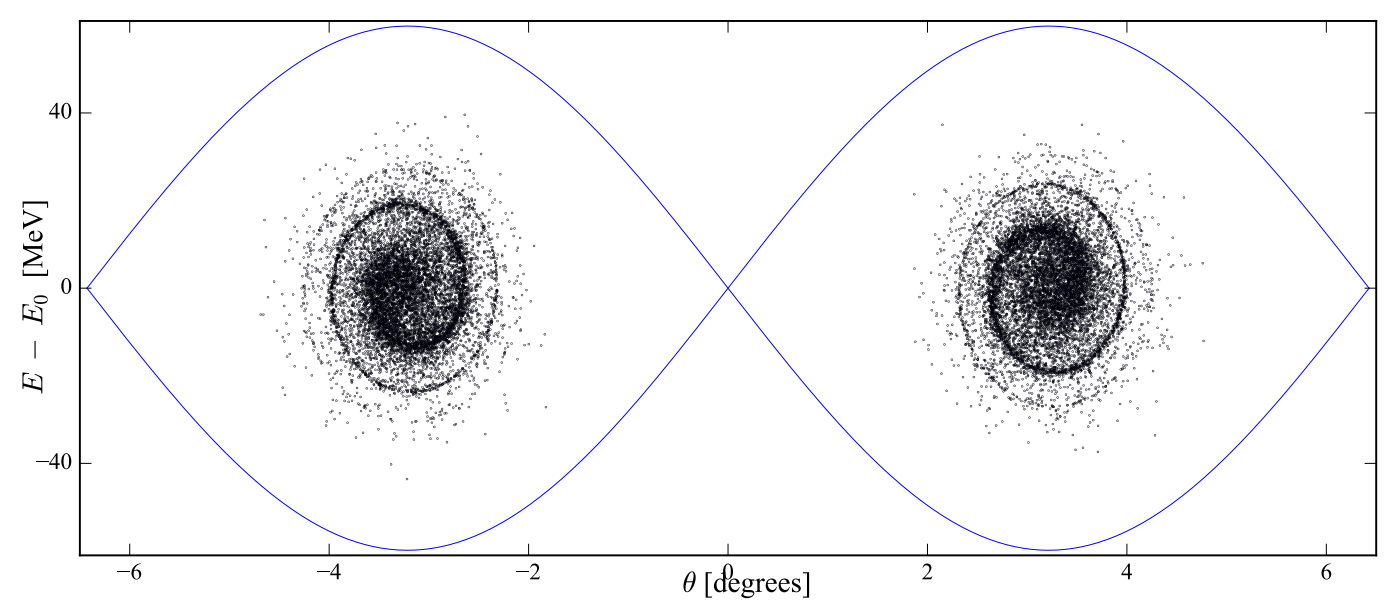

FIG. 49: Bunch splitting for $T_{\text {split }}=10 T_{\mathrm{s}}$ showing the decoherence of the bunches.

The current formation strategy describes injecting 26 bunches at $h=28$ into the ion collider ring which is then used to split evenly seven consecutive times. To continue with the rest of the splits, the splitting time was kept to a constant $10 T_{\mathrm{s}}$ for each individual splitting process, where the synchrotron period $\left(T_{\mathrm{s}}\right)$ is the lowest out of the two RF systems used in the split.

TABLE 16: Harmonic number and the corresponding amplitude for the whole RF systems used for the complete splitting process.

\begin{tabular}{lc} 
Harmonic & RF amplitude $[\mathrm{kV}]$ \\
\hline 28 & 10 \\
56 & 20 \\
112 & 40 \\
224 & 80 \\
448 & 160 \\
896 & 320 \\
1792 & 640 \\
3584 & 1280 \\
\hline
\end{tabular}

Fig. 50 shows the RF amplitude variation through time for the eight RF systems used for the complete process of splitting one bunch at $h=28$ to 128 smaller bunches at $h=3584$. 


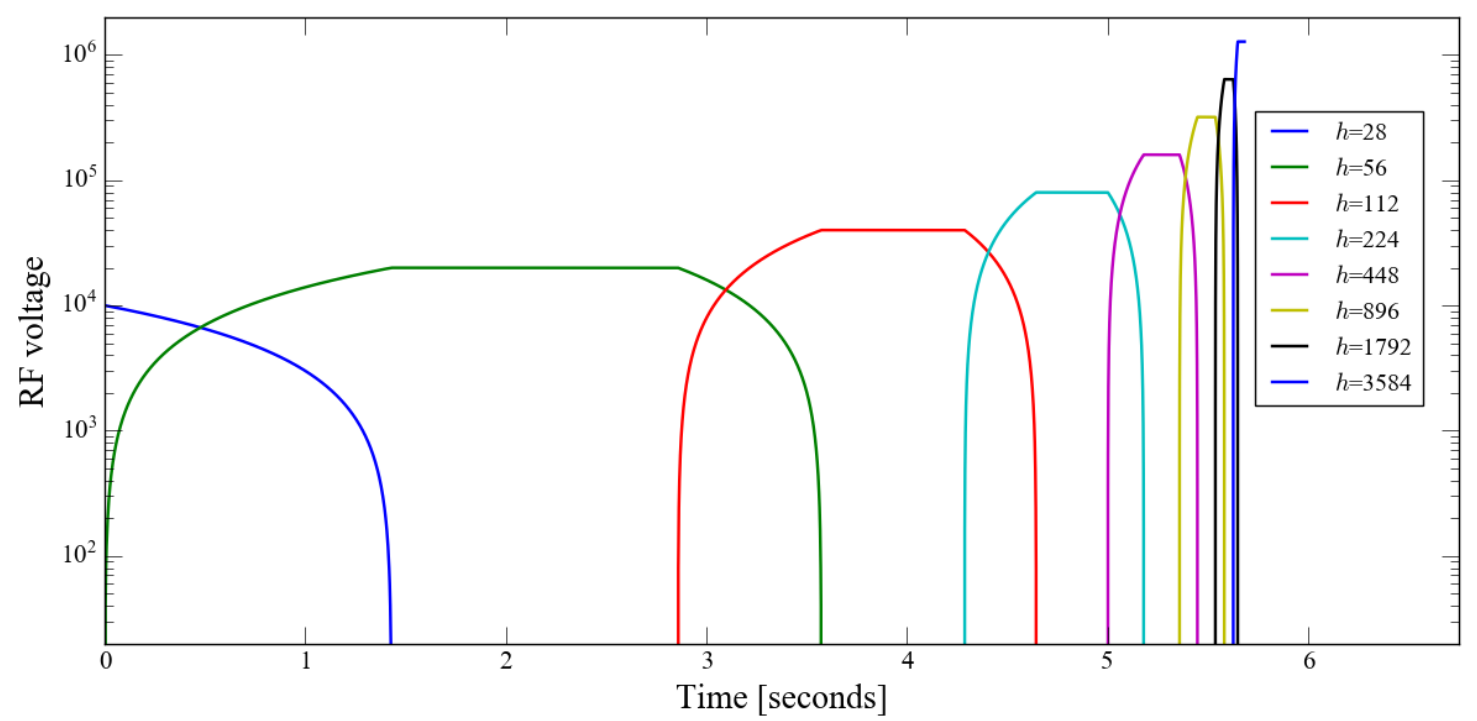

FIG. 50: RF voltages (on a log scale) for the different harmonics used in the complete splitting process as a function of time.

The resulting formation of the 128 bunches after the 7 consecutive splits is shown in Fig. 51

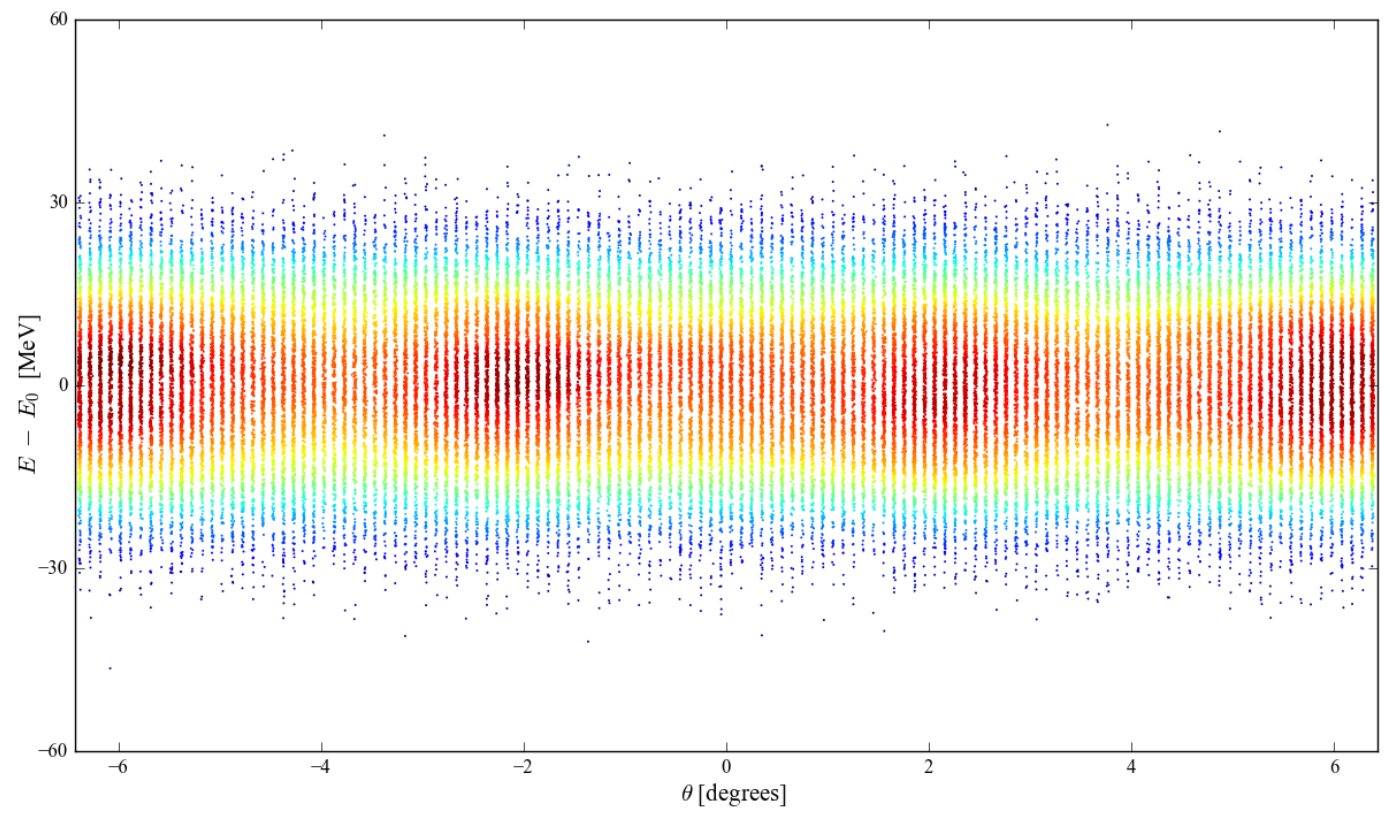

FIG. 51: Final Bunch formation with $T_{\text {split }}=10 T_{\mathrm{s}}$ for each split.

This final bunch formation shows an uneven emittance spread which is a result from the decoherence that occurs from the splitting process being not adiabatic enough with a split 
time of 10 synchrotron periods. This result shows that a better understanding is needed about the split time to find an optimum value with acceptable emittance growth.

\subsection{PARAMETER SCAN}

Selecting a split time $T_{\mathrm{s}}$ based on the adiabaticity parameter alone does not give a precise value for $T_{\mathrm{s}}$. The parameter only acts as a constraint according to the given tolerance for the emittance growth. So a parameter scan was done to gain better knowledge of the splitting time $T_{\text {split }}$, which must be long enough to make the process effectively adiabatic and preserve longitudinal emittance within desired tolerances. However, having unnecessarily longer split times are not practical either due to instabilities acting on the bunches. So a parameter scan was performed with the two obvious parameters in the splitting process, the split time (taken as the time duration of the voltage ramps, which at the moment are equal to each other) and the bunch to bucket ratio. For $h=28$ and $\mathrm{RF}$ voltage $V_{\mathrm{rf}, h}=28=10 \mathrm{kV}$ in [7], $T_{\mathrm{s}} \equiv T_{\mathrm{s}, h}=28=18796$ turns or $138 \mathrm{~ms}$.

The splitting RF voltage is given by assuming bucket areas scale as they split. This gives $V_{\mathrm{rf}, h=56}=2 V_{\mathrm{rf}, h=28}$, preserves bucket height, and gives $T_{\mathrm{s}, h=28}=2 T_{\mathrm{s}, h=56}$. With these parameters, ESME simulations were performed of 1:2 splits with split times $T_{\text {split }} / T_{\mathrm{s}}$ ranging from 10 to 100, and the initial total bunch emittances ranging from $2.5 \%$ to $50 \%$ of the RF bucket. This gives information to optimize split time for a given initial longitudinal emittance and emittance growth tolerance. Results are shown in Fig. 52 and Fig. 53. Noted are the different emittance growth factor scales, where the color bar scaling is the ratio of final emittance of both bunches to the emittance of the initial bunch. A value of 1.0 indicates a perfect split with no net emittance growth. The results of the parameter scan was divided into two categories with one having the smaller bunch to bucket ratio with split times less than $30 T_{\mathrm{s}}$ and the other having a larger bunch to bucket ratios and split times larger than $30 T_{\mathrm{s}}$. This gave a better resolution of the results. 


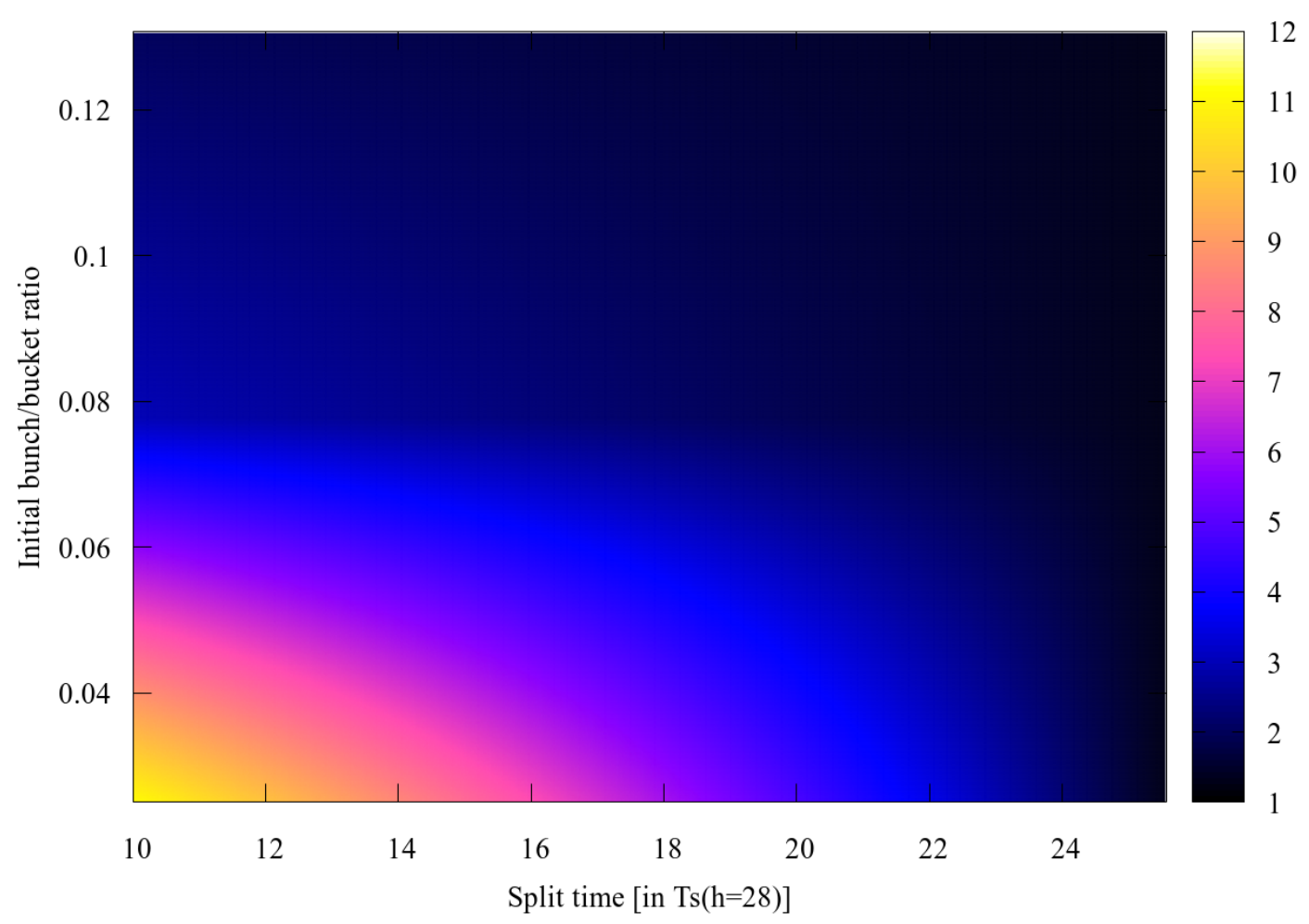

FIG. 52: JLEIC 1:2 split emittance growth, $T_{\text {split }} / T_{\mathrm{s}}<30$.

Fig. 52 shows that split times of 10 to $30 T_{\mathrm{s}}$ create considerable longitudinal emittance growth unless the initial bunch is over $10 \%$ of the bucket area. Naive expectations might consider this split time to be "adiabatic enough", but even split times of $30 T_{\mathrm{s}}$ cause more than $20 \%$ emittance growth per split. This is not a product of the lower harmonic choice $h=28$ for $T_{\mathrm{s}}$, as $30 T_{\mathrm{s}, h=28}=60 T_{\mathrm{s}, h=56}$ here. It seems that the emittance growth from having a lower bunch to bucket ratio could be compensated by the fact that the synchrotron period for the same bunch size goes up by using a higher RF voltage. In this way the splitting process can take the same amount of time but with a higher number of synchrotron periods. But as seen from the previous section the decoherence caused by the low bunch to bucket ratio causes uneven splits in the future as the process continues. 


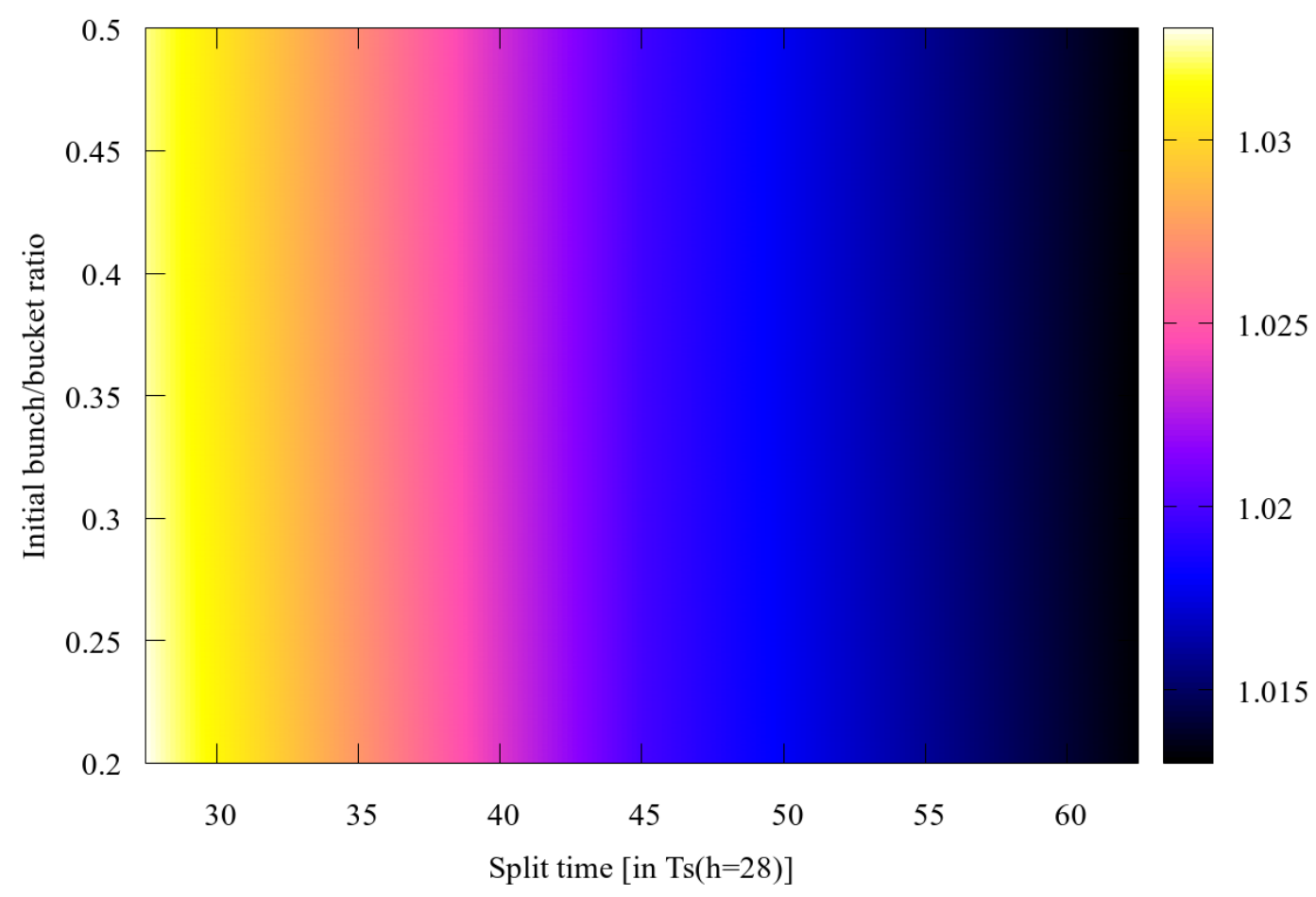

FIG. 53: JLEIC 1:2 split emittance growth, $T_{\text {split }} / T_{\mathrm{s}}>30$.

Fig. 53 shows that split times of $50 T_{\mathrm{s}}$ or more are needed to constrain emittance growth to below $1 \%$ per split. This result appears robust against a wide variety of initial bunch emittances, and will be used to preserve emittance growth for simulations of multiple bunch splits. It has significant implications for simulation time with realistic distributions. It is important to note that below $1 \%$ emittance growth the computational errors from tracking has significant effect on the emittance calculation resulting in an inaccuracy that is larger than the calculated emittance growth. From the parameter scan it was shown that it is possible to speed up the splitting process by increasing the RF amplitude which decreases the synchrotron period by $\sqrt{V_{R F}}$, but this results in lowering the bunch to bucket ratio since the bunch sizes are limited by design. 


\subsection{MULTIPLE 1:2 SPLITTING FOR JLEIC}

Using the results from the parameter scan a more suitable split time of 50 synchrotron periods was selected. It was also observed that as the split time goes up, the decoherence decreases giving a better overall splitting. The results from the initial split for 50 synchrotron periods is shown in Fig. 54 .

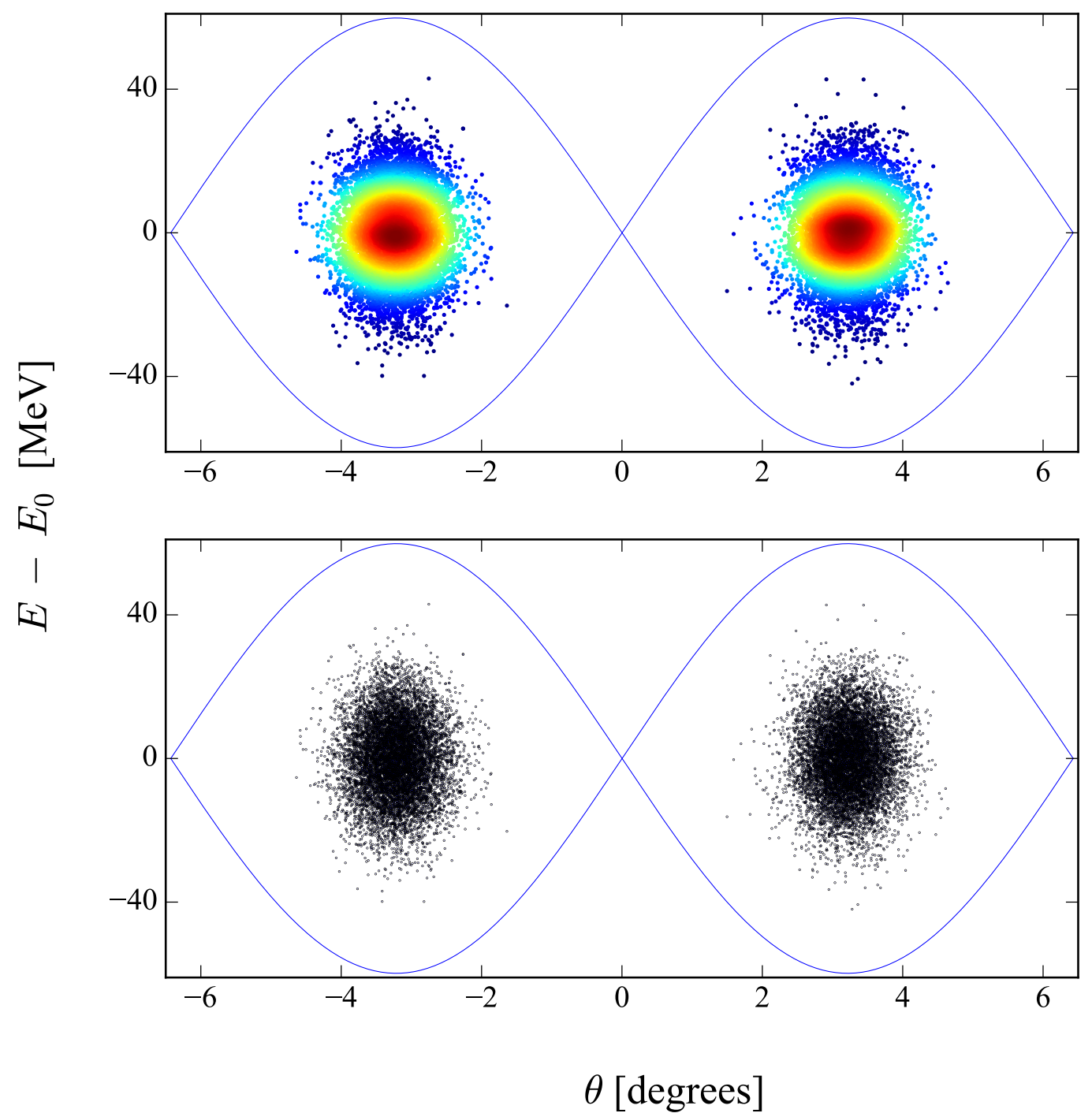

FIG. 54: Bunch formation with $T_{\text {split }}=10 T_{\mathrm{s}}$ for the initial split with the density heat map. 


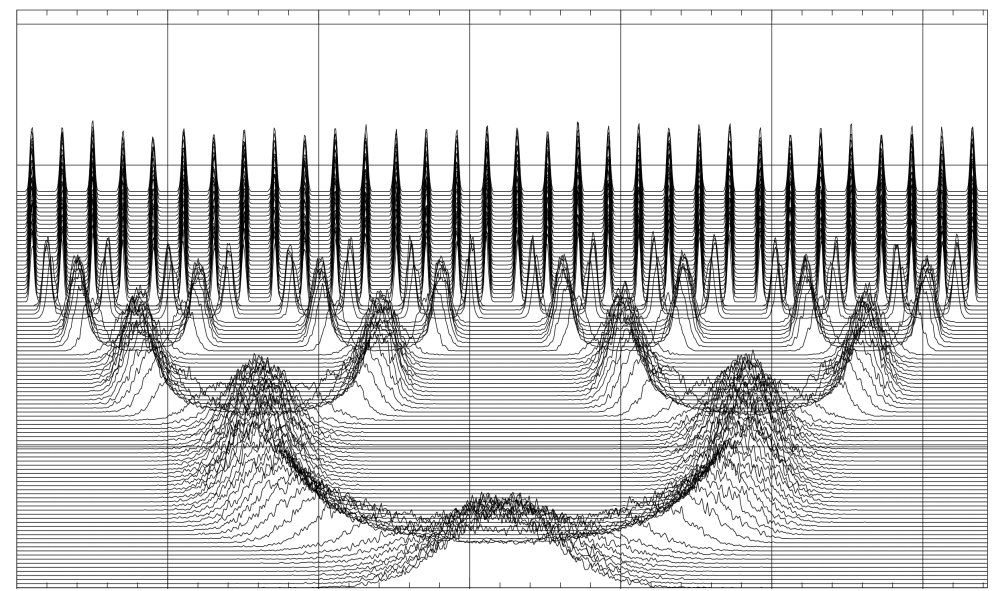

(c)

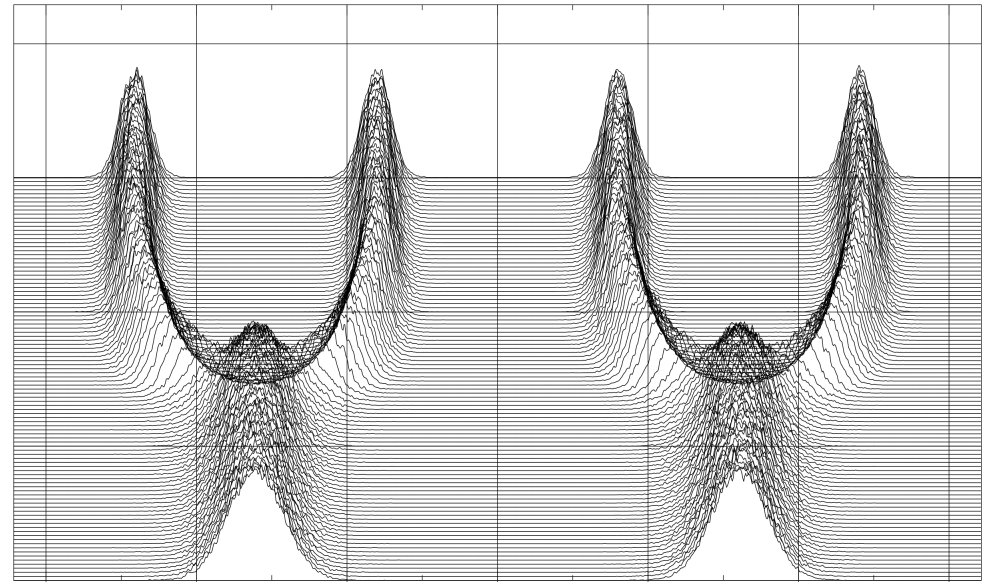

(b)

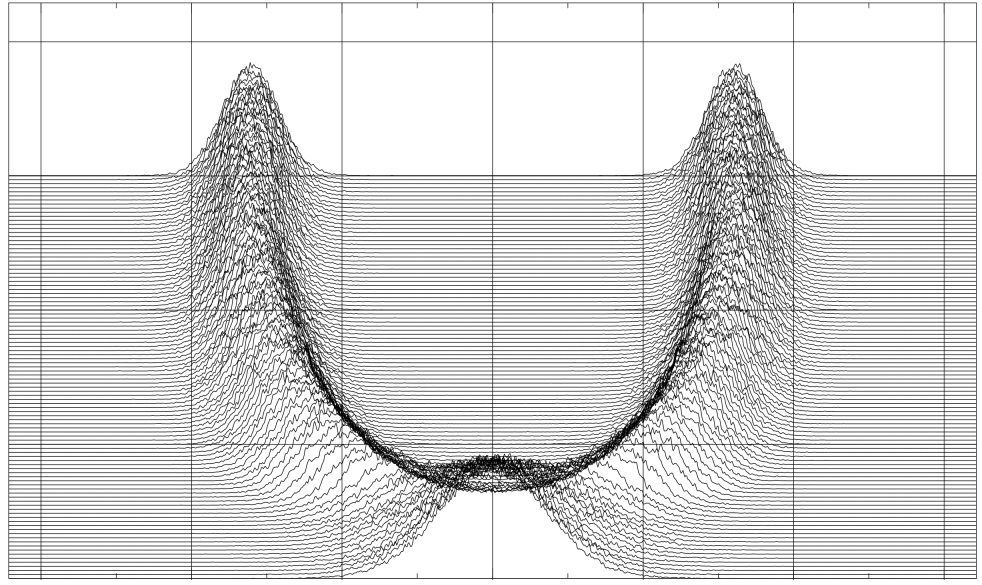

(a)

FIG. 55: Bunch current profile of the splitting process. Each line is plot with $\sim 1 \mathrm{~s}$ time difference. (a) shows the initial split and (b) shows the second split. (c) shows rest of the five remaining splits for one bunch out of the four in (b). 
The bunches after the split did not show the decoherence as much as seen when splitting with $T_{\text {split }}=10 \mathrm{~s}$. They were more symmetric and preserved the longitudinal emittance compared to the simulation with a split time of 10 synchrotron periods. The final bunch formation obtained by continuing this splitting process for 6 more splits ( 7 in total) is shown in Fig. 56.

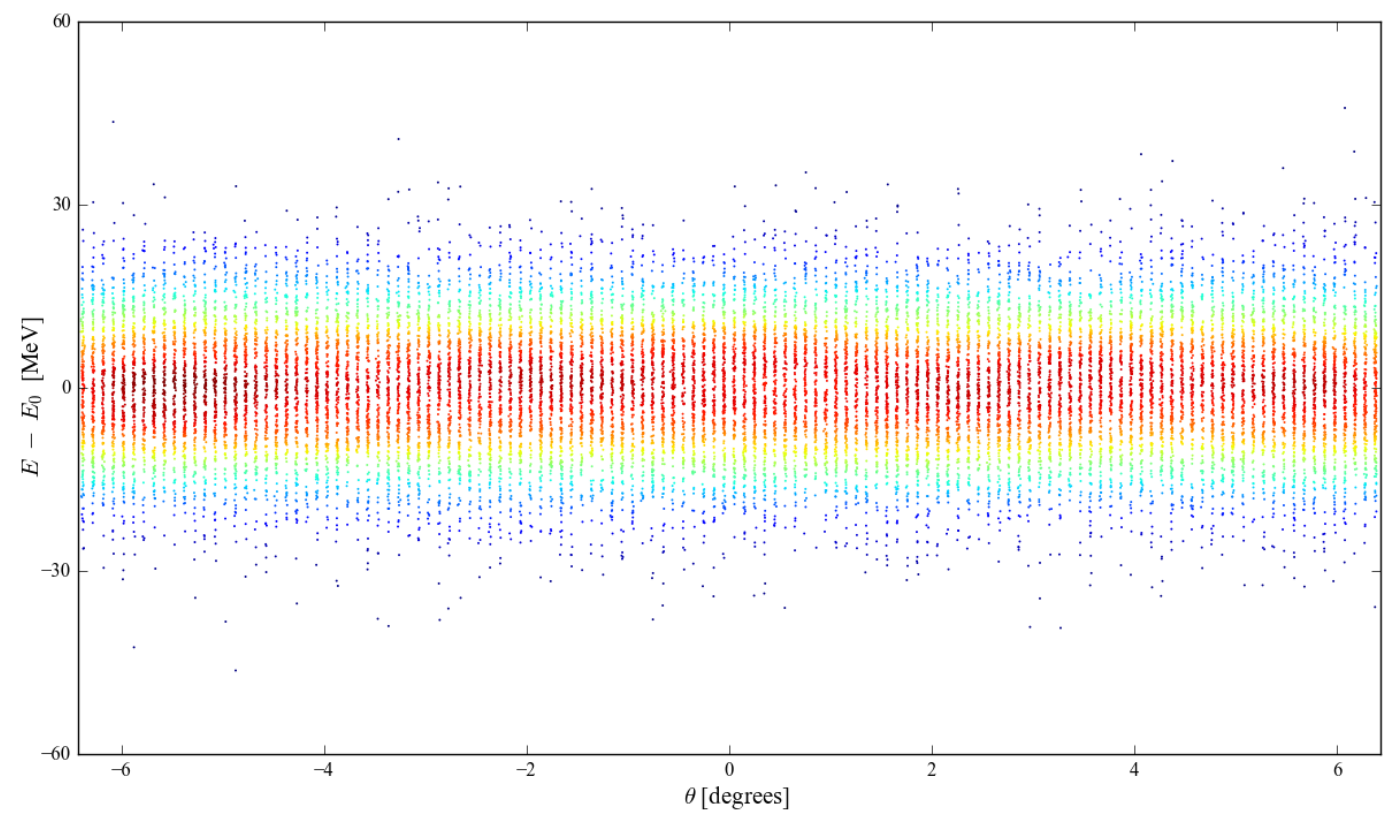

FIG. 56: Final Bunch formation with $T_{\text {split }}=50 T_{\mathrm{s}}$ for each split.

Because the number of synchrotron periods per split, the harmonic relation, and RF amplitudes ratios kept the same for all consecutive splits, it is safe to assume that the emittance growth is consistent throughout the entire splitting procedure. So using Eq. 106 the total emittance growth can be calculated.

$$
\epsilon_{g}^{T}=\left(1+\epsilon_{g}^{S}\right)^{n}-1
$$

where $n$ is the number of splits, $\epsilon_{g}^{T}$ is the total emittance growth and $\epsilon_{g}^{S}$ is the emittance growth for a single split. For an emittance growth of $1 \%$ per single split, the calculated total growth is $7.21 \%$. For the current simulation with an emittance growth of below $1 \%$ for each split the total emittance growth of the resulting bunch formation was observed in the range $3 \%$ which is significantly below the $10 \%$ emittance growth tolerance for JLEIC. ESME provides a good estimate for the emittance growth but for a more precise estimate of the emittance (under 1\% per split) growth for the whole process, a different simulation code with more precision is required. 


\section{CHAPTER 7}

\section{BUNCH SPLITTING OPTIMIZATION}

The bunch splitting process has more parameters than just the bunch to bucket ratio and the split time discussed in the previous chapter. Altering these parameters can result in hastening the splitting process. For example, increasing either the voltage or the harmonic number increases the synchrotron tune (Eq. 57), which means shorter periods for the synchrotron oscillations. This speeds up the splitting process since the split time is measured in synchrotron periods. But increasing these parameters may not always be a possibility. For example, for a given bunch size, increasing the RF amplitude will result in a low bunch to bucket ratio which blows up the emittance of the final bunch as shown in section section 6.4. This chapter investigates if the splitting process time can be minimized by adding more parameters to the variation, including but not limited to the voltage variations of the process. With each additional parameter, the space to scan for solutions increases such that for $n$ parameters the search space becomes $n$-dimensional. Performing linear scans of the parameter space at this point is computationally expensive and so other strategies must be used.

One family of optimization strategies that can handle $n$-dimensional search spaces are genetic algorithms (GAs), which are initialized with a population of models that undergo selection, recombination, and mutation across subsequent generations to locate optimal solutions. In this chapter, the bunch splitting parameter space is investigated with GA optimization using the code PISA [49] with modifications by Dr. Balsa Terzic [50] [51] to find solutions that may further expedite the splitting process. The theory of GAs is discussed, including the processes by which the fittest individuals are selected for propagation and populations are evolved through the sharing of genetic material and random mutation. Specific parameters are then introduced and results are shown for a GA optimization for JLEIC bunch splitting that aims to minimize both splitting time and emittance growth to avoid blowout.

\subsection{GENETIC ALGORITHMS}

When it comes to $n$-dimensional parameter searches, evolutionary computation plays a major role in finding the optimal solutions. A subset of this is genetic algorithms (GAs), 
which are based on the evolutionary concepts of natural selection and genetics. By combining random search with historical data they significantly improve performance within the search space by simulating the evolution of a biological population.

In such simulations, a population is a selection of points in the variable space, one iteration of which composes a generation. Individuals are composed of sets of independent variables, or genes, which vary in a granular manner among individuals. The algorithm searches for the optimal solution by iterating through generations of candidate solutions where the best candidates are selected based on their fitness, or their proximity to the optimal solution. These best candidates undergo propagation, wherein genes are randomly passed on to offspring from each parent through the process of recombination, and random mutation to make up the next generation. The selection and propagation of the selected individuals is as follows:

1. individuals in a population compete for resources and mates,

2. the individuals who are most successful in each competition will produce more offspring than those that did not,

3. an offspring produced by two good parents will be better than either parent.

This process (Fig. 57) results in the propagation of beneficial genes throughout the second generation and in each successive generation being more suited for the environment than the last. In this way, the GA simulates the evolution via natural selection of the objective function, which is the equation containing all of the considered parameters and for which the goal is, in this particular case, minimization of the split time and emittance growth. What follows in this section are in-depth descriptions, definitions, and processes of GAs. 


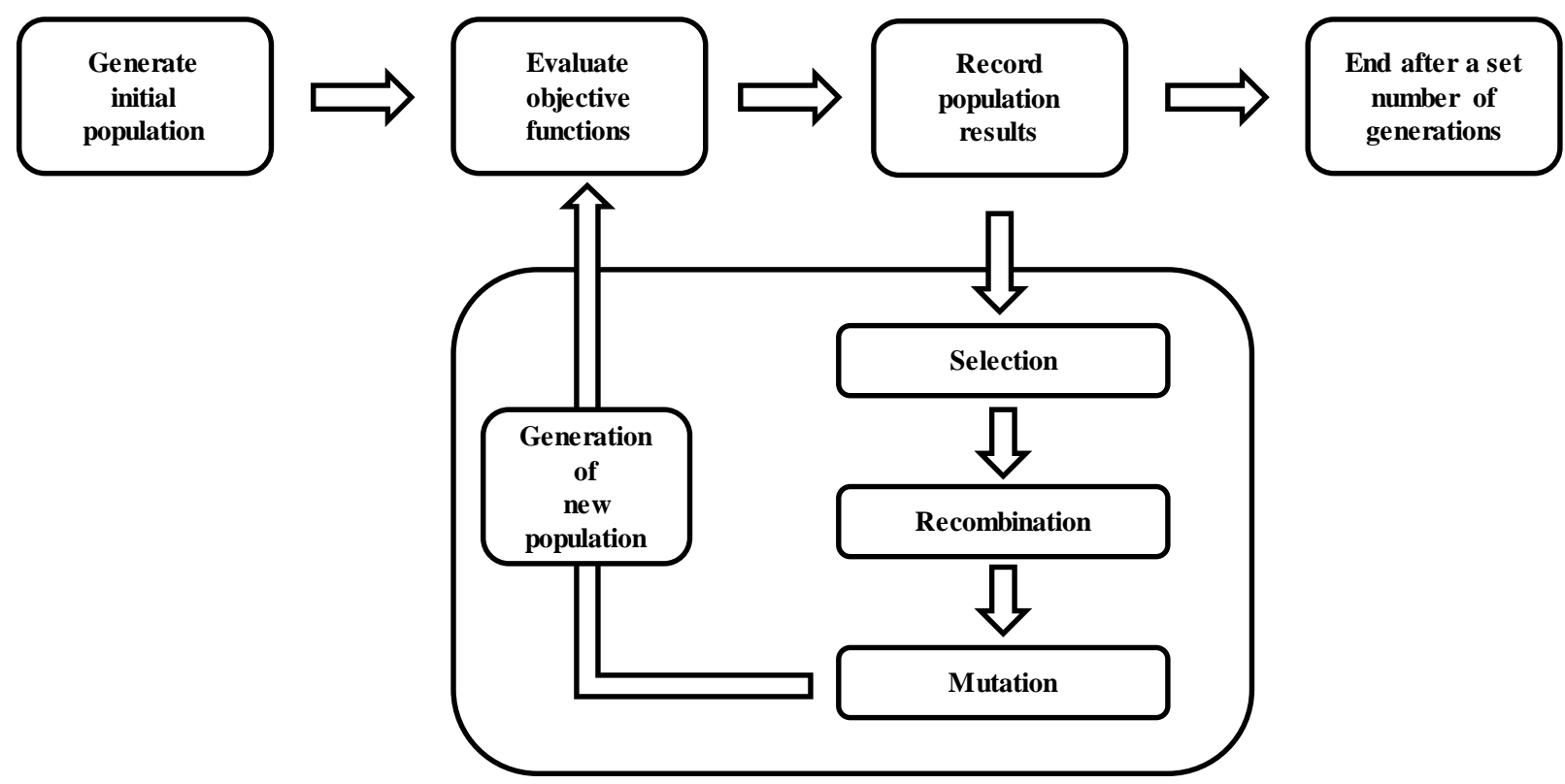

FIG. 57: Flow chart for the genetic algorithm.

\subsubsection{COMPETITION AND DOMINANCE}

Before the evolution of a population can be simulated, it must first be populated with individuals that randomly span the search space, in order to avoid the focusing of the selective process onto a local optimum. The population size should be set at a value large enough to allow for sufficient genetic variety and drift across the search space without converging too quickly, while also being small enough to successfully converge without the use of an unrealistic number of evaluations. Following the creation of a population, feasible individuals, or sets of values that meet all requirements of the bounded domain of the optimization, compete with one another for dominance. For a multi-objective minimization query, an individual with a more ideal value for the objective function than another individual is dominant, as explained further in Section subsection 7.1.4. In this instance, the dominant individual would be selected from the population for the mating pool, with other dominant individuals, in order to propagate the next generation. However, multi-dimensional problems may introduce conflicting objectives, where variables influence each other inversely and cause the presence of multiple equally optimal solutions.

\subsubsection{RECOMBINATION AND MUTATION}


In the mating pool, composed of the dominant individuals from a generation, parents undergo recombination of their variable sets, or genes, to produce offspring. Recombination is the process through which one of the parents' variable values is randomly chosen for each variable independently of the others, or where offspring values are a function of the values of that variable in both parents. In addition to selection of the independent variable values from parents, some variables also undergo random mutation, where they generate a new value not present in the parental value sets.

\subsubsection{EVOLUTION OF POPULATION, FITNESS}

An individual may enter the mating pool multiple times based on how many competitions it wins, a result of its fitness. Fitter individuals having been entered into the mating pool more times than less fit individuals and will have greater chances to reproduce, resulting in the propagation of their genes in greater abundance in the offspring generation. Over subsequent generations, the characteristics of the population migrate toward the optimal solution through this natural selection, and the overall fitness of the population improves.

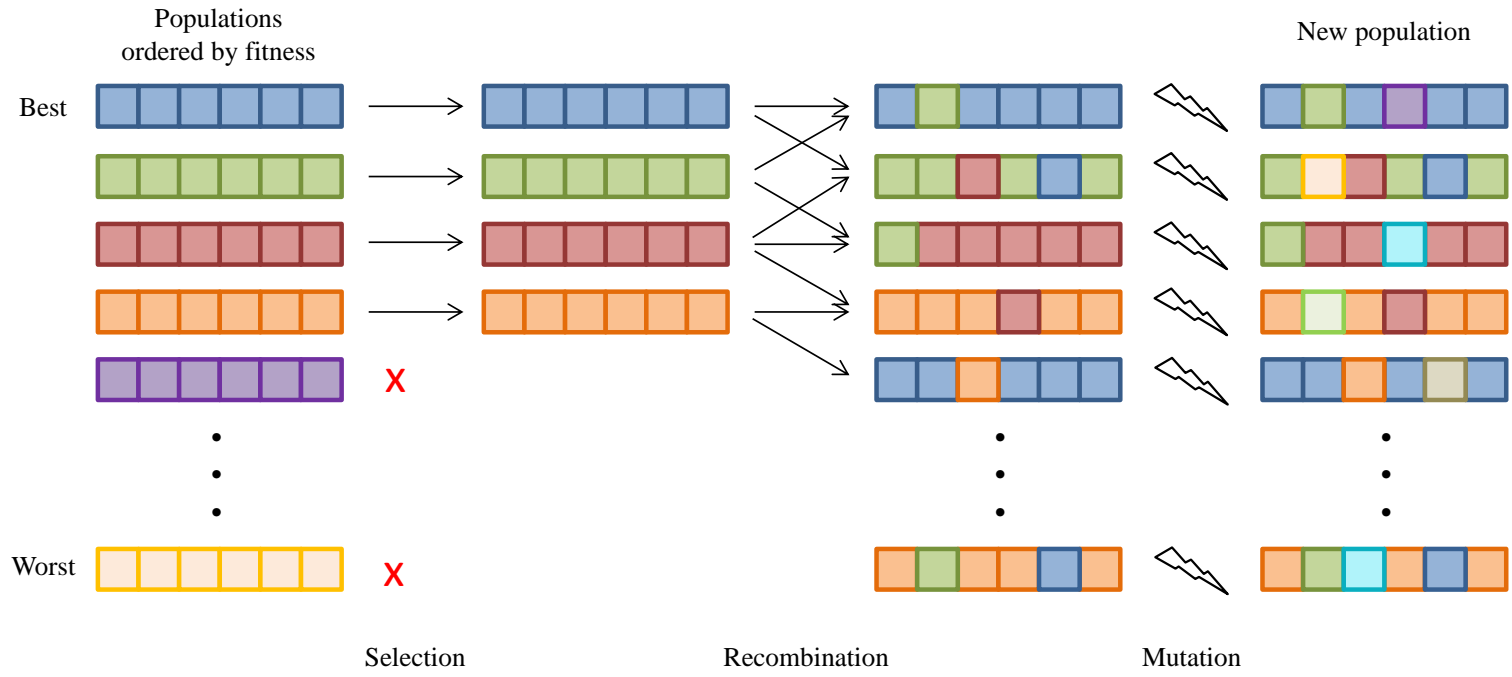

FIG. 58: Diagram of the population advancing to the next generation. 


\subsubsection{PARETO OPTIMAL FRONT}

The solutions gained through the GA after a specified number of generations for a twoobjective problem, in this case the minimization of split time and emittance growth, can be further examined on a Pareto-optimal front, a fitted curve drawn through all the most dominant points on each single-objective optimization, as shown in Fig. 59. A point in the solution space is said to be dominant if there are no other solutions that fall inside the rectangular box defined by its $x$ and $y$ coordinates. For example, in Fig. 59, point $\mathrm{C}$ is non dominant because point $\mathrm{A}$, representing a fitter individual, contains lower values for split time and/or emittance growth and thus falls within the rectangular boundary of point C. Points A and B are both dominant because they do not fall within boxes defined by any of the other points. At extremes of each axis the curve represents the corresponding single-objective optimization. The section between these extremes gives a trade-off gradient between them. in Fig. 59, both points $\mathrm{A}$ and $\mathrm{B}$ would qualify as solutions to the minimization problem, but the user may choose one solution over the other based on the needs and limitations of the system. For the JLEIC bunch splitting, a solution for minimum split time could be chosen given the allowed emittance growth tolerance.

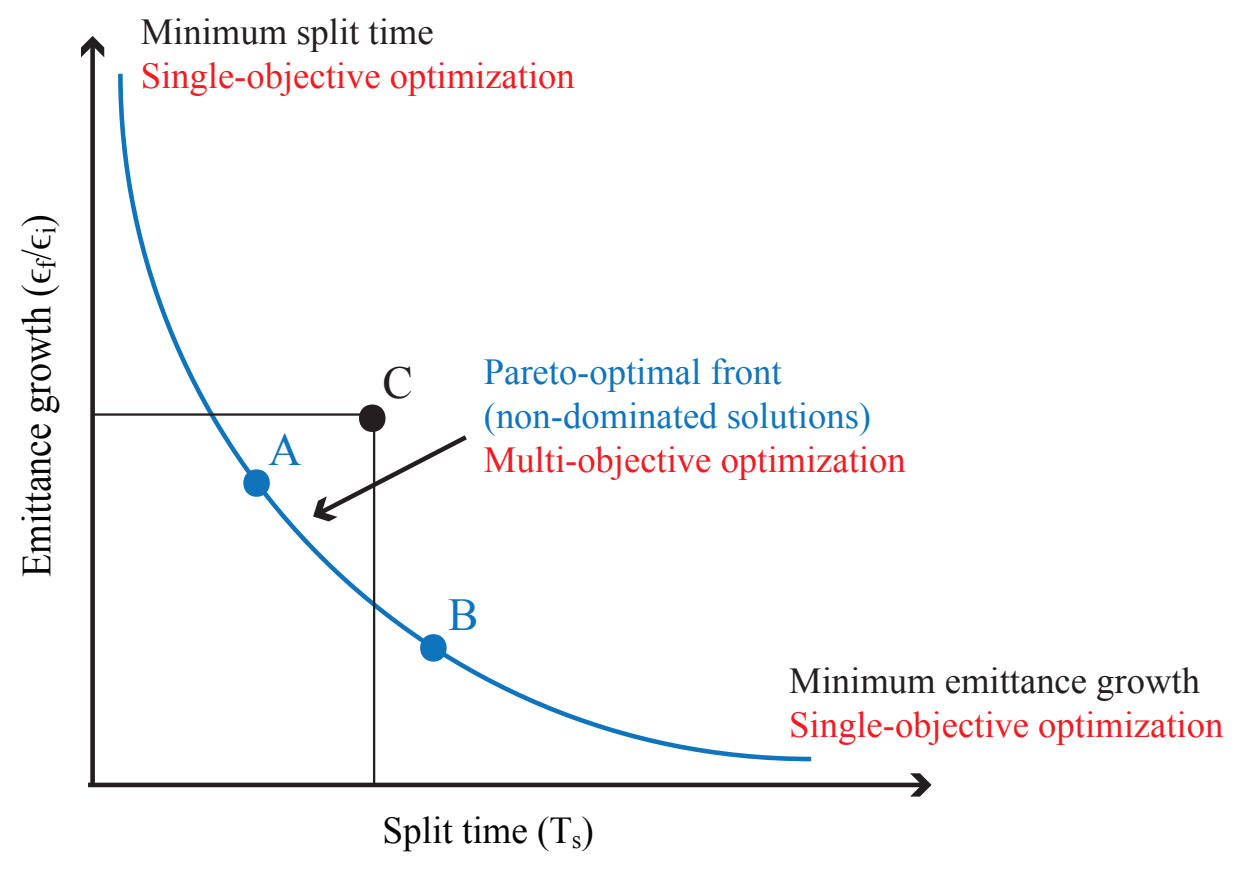

FIG. 59: Pareto optimal front. 


\subsection{PARAMETERS FOR THE GENETIC ALGORITHM}

The additional parameters that were considered for the GA optimization are shown in Fig. 60 and are defined as,

- $V_{\mathrm{RF} 1}$ : Maximum voltage amplitude of the RF source 1

- $V_{\mathrm{RF} 2}$ : Maximum voltage amplitude of the RF source 2

- T1BEG : Beginning time for the RF source 1 voltage ramp

- T1END : Ending time for the RF source 1 voltage ramp

- T2BEG : Beginning time for the RF source 2 voltage ramp

- T2END : Ending time for the RF source 2 voltage ramp

- $\mathrm{T}_{\text {split }}$ : Total time for the splitting process

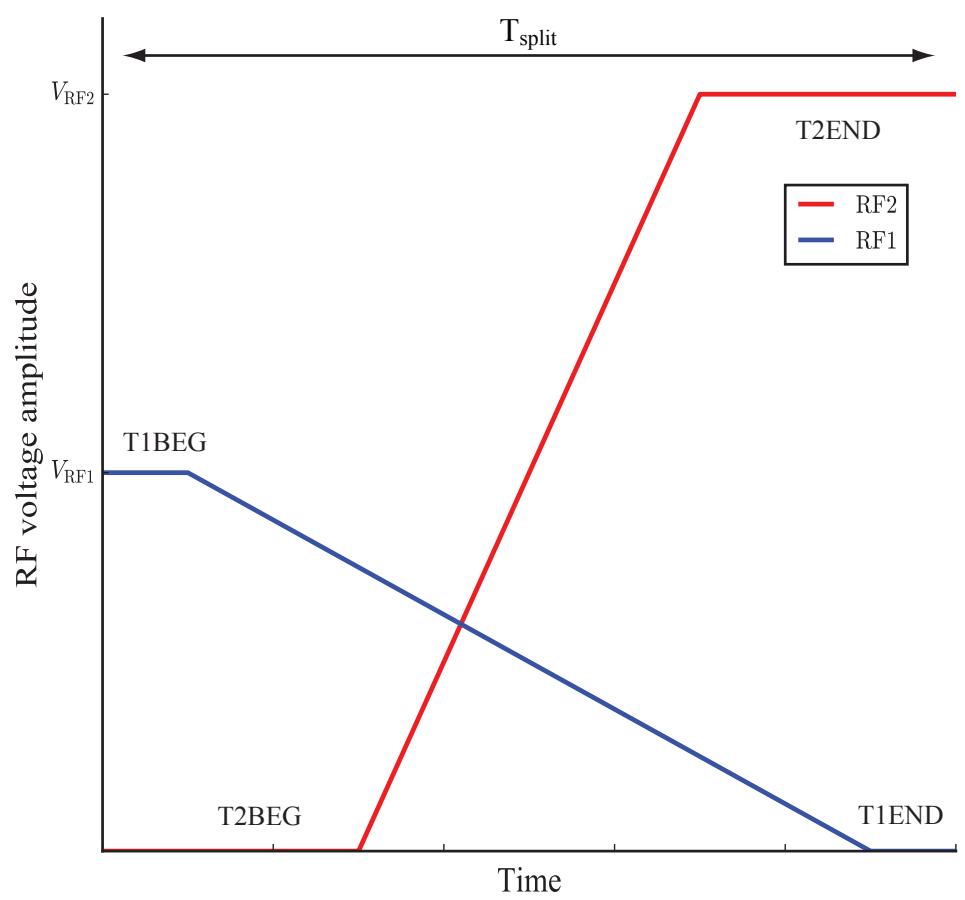

FIG. 60: Variables selected for the GA optimization. 
TABLE 17: GA Optimization cases.

\begin{tabular}{|c|c|c|c|}
\hline RF ramp type & Bunch to bucket ratio & Figs & $\mathrm{T}_{\text {split }}$ for $1.0137 \%$ growth \\
\hline Linear & 0.3 & Fig. 61 and Fig. 66 & 22 \\
\hline Linear & 0.05 & Fig. 63 and Fig. 67 & 85 \\
\hline Iso-adiabatic & 0.3 & Fig. 68 & 60 \\
\hline Iso-adiabatic & 0.05 & Fig. 69 & 80 \\
\hline
\end{tabular}

The parameters chosen for the GA simulations vary depending on the problem. But the four time parameters were kept common for all. They are $T 1_{B E G}, T 1_{E N D}, T 2_{B E G}, T 2_{E N D}$ with constraints

$$
\begin{aligned}
T 1 \mathrm{BEG}, T 2 \mathrm{BEG} & \leq T 1 \mathrm{END} \\
T 2 \mathrm{BEG} & \leq T 2 \mathrm{END} \\
T 2 \mathrm{END}, T 2 \mathrm{END} & \leq T \text { total. }
\end{aligned}
$$

The time constraints impose the conditions to keep the two ramps intersecting with each other and not create a time interval where there is no RF control on the bunches. With no RF control the particles will start to drift freely in the beam pipe and will not be completely captured by the final RF system. Running a large number of simulations is required to gain better results. To gain faster results with less computational time, the splitting was done at a higher harmonic. The initial values selected for this simulation are shown in Table 18 . 
TABLE 18: RF system parameters used in the GA simulation.

\begin{tabular}{lcc}
\hline \hline Harmonic & RF amplitude $[\mathrm{kV}]$ & \\
\hline$T_{\text {total }}$ range & $0-0.8$ & {$[\mathrm{~s}]$} \\
$V_{R F 1}$ & 160 & {$[\mathrm{kV}]$} \\
$h_{1}$ & 224 & - \\
$V_{\mathrm{RF} 2}$ range & $160-320$ & {$[\mathrm{kV}]$} \\
$h_{2}$ & 448 & - \\
$\epsilon_{i}$ & 0.48 & $\mathrm{eV}-\mathrm{s}$ \\
\hline \hline
\end{tabular}

With the selected initial values and constraints, the objective of the genetic algorithm was to minimize the longitudinal emittance growth of the splitting process.

\subsection{RESULTS FROM THE GENETIC ALGORITHM}

The GA optimization's were all performed for two cases. One with a bunch to bucket ratio of 0.3 and the other for a much smaller ratio of 0.05 . This gives a comparison of the low and high ends of the starting bunch emittances.

\subsubsection{LINEAR VOLTAGE CURVES WITH VARIABLE RAMPING TIMES AND A CONSTANT VOLTAGE RATIO}

For this simulation the variables $T 1_{B E G}, T 1_{E N D}, T 2_{B E G}$, and $T 2_{E N D}$ were varied and the voltage ratio was kept constant at $V 2 / V 1=2$. This keep the energy spread of the resulting bunches the same as the energy spread of the initial bunches since the ratio $h / V$ is kept constant. The voltage ramps are linear in time. In this run each GA simulation in ESME had a population of 60 individuals and 150 generations totaling to 9000 total simulations. The JLEIC tolerance for the whole process is $10 \%$. This makes the tolerance for one split to be $1.37 \%$ (or 1.0137 emittance ratio in the figures). The Pareto-optimal front for the bunch to bucket ratio of 0.3 is shown in Fig. 61 . 


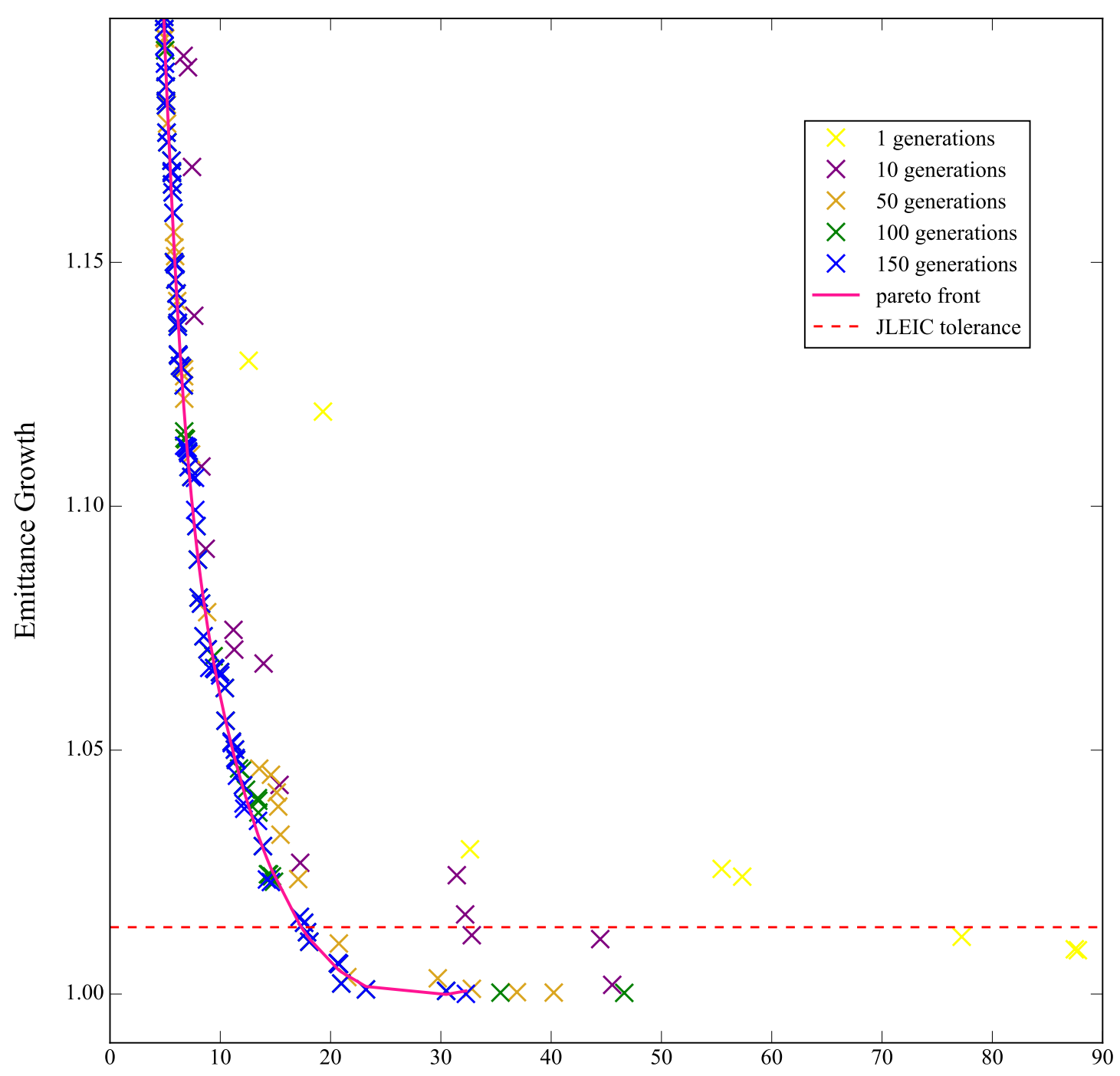

Split Time [Synchrotron periods]

FIG. 61: Pareto optimal curve for linear voltage variation with a bunch to bucket ratio of 0.3 .

It was observed that with a splitting time of 15-20 synchrotron periods, an emittance growth of as low as $1 \%$ is achieved per split. This is in contrast to a splitting time of 50 synchrotron periods, identified as the minimum necessary time to achieve this emittance growth level in the previous chapter. It is observed that overall time for a splitting process can be lessened while achieving the same results using the GA. It is also clear from the plot that the data points settle on the Pareto-optimal curve after as few as 50 generations of the GA, but marginal improvements are achieved with more generations. An example data 
point taken from the Pareto front was used to create the longitudinal phase space plot seen in Fig. 62.

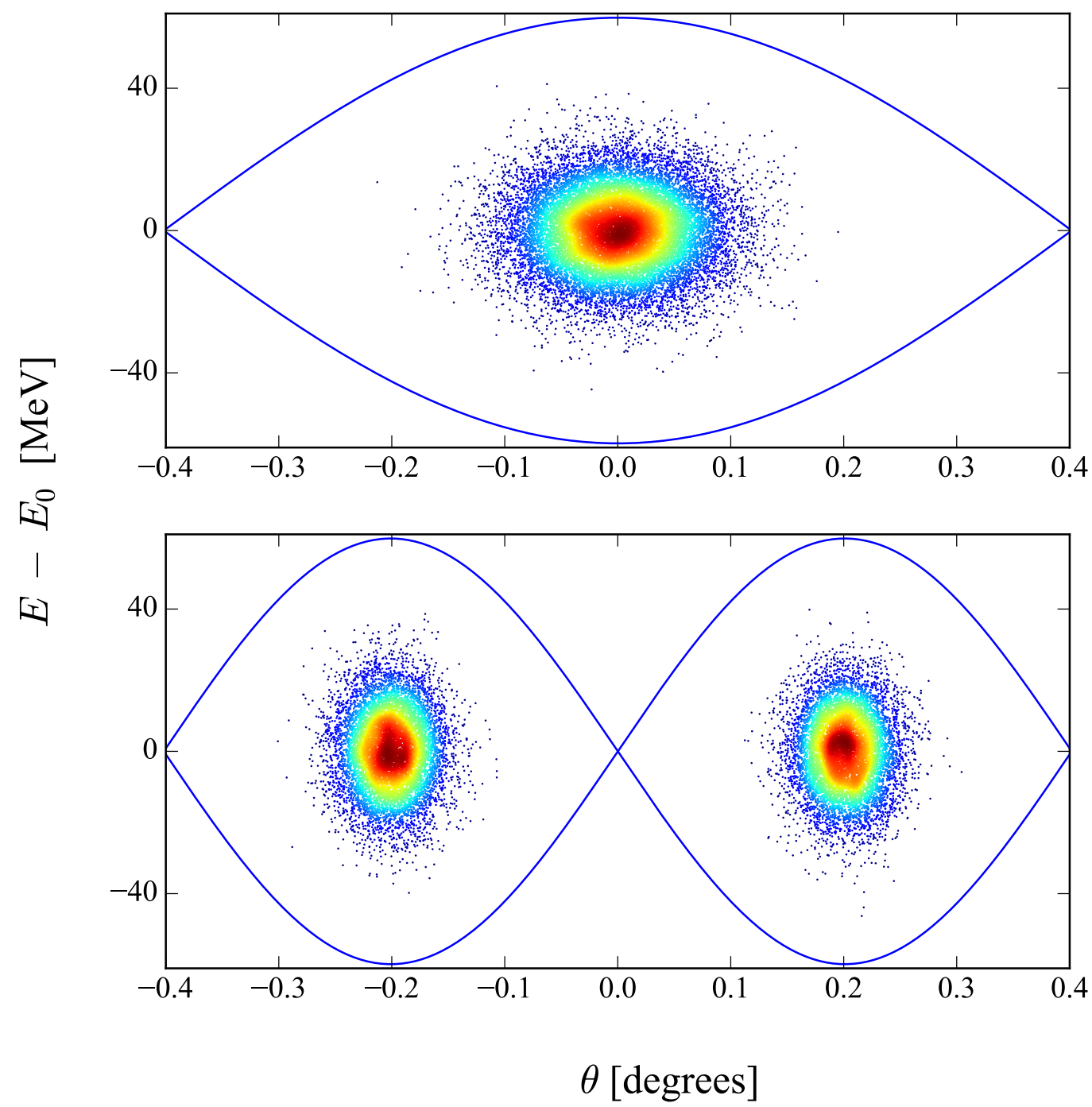

FIG. 62: Bunch splitting for $T_{\text {split }}=25 T_{\mathrm{s}}$ using the results from Fig. 61. Emittance growth was below $1 \%$.

An individual inspection of the data suggests more favorable results with minimum emittance growth and short splitting times were obtained when the final RF source ramps up before the initial RF source ramps down as seen in Fig. The second run is for a bunch to bucket emittance ratio of 0.05 . For this run a longer split time is required (more than $80 \%$ ) compared to the first case, to get an emittance growth closer to $1 \%$ per split. 


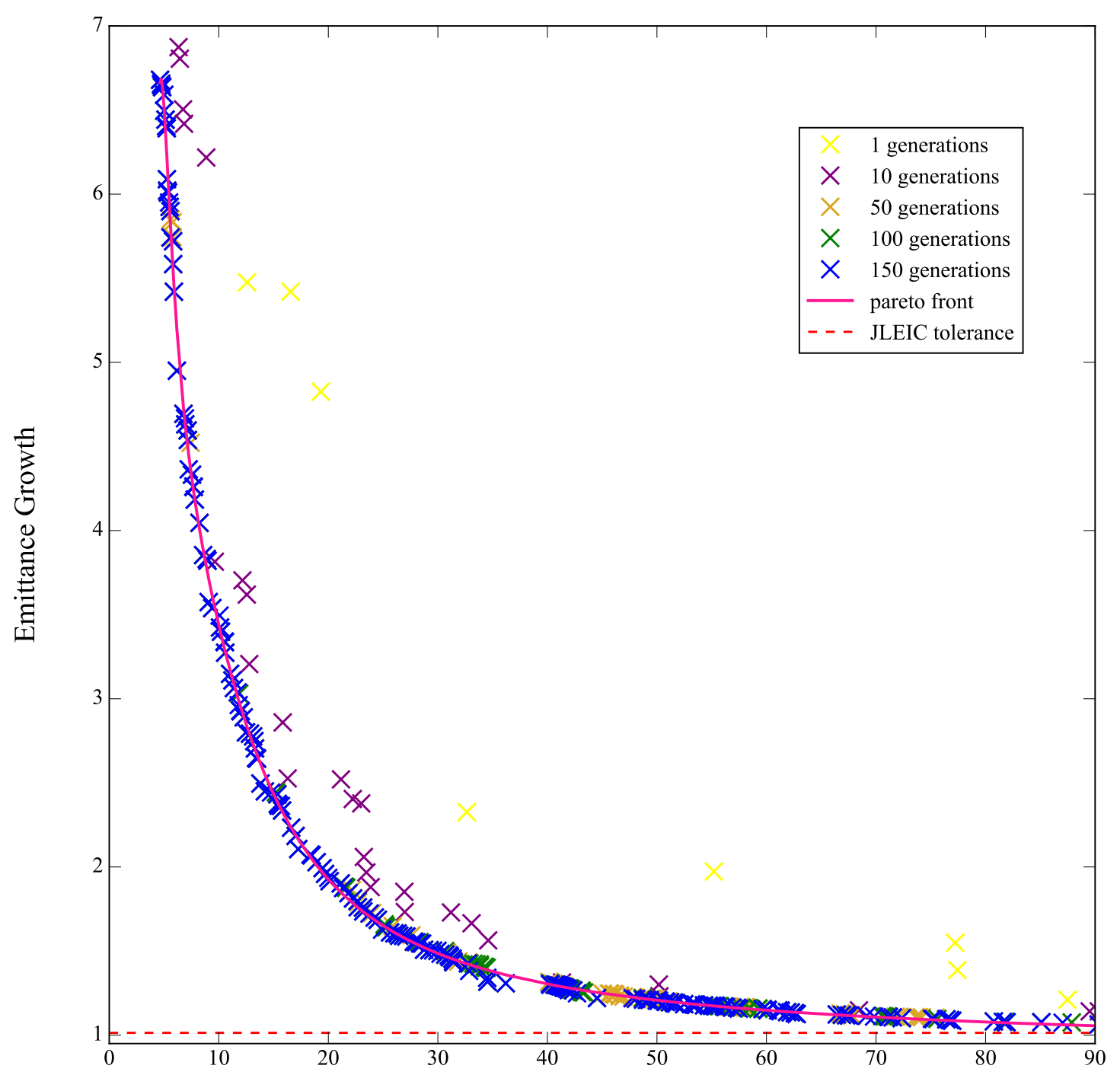

Split Time [Synchrotron periods]

FIG. 63: Pareto optimal curve for linear voltage variation with a bunch to bucket ratio 0.05.

The fitted curve did not intersect the JLEIC threshold line of 1.0137 emittance growth. A sample data point was selected at 85 synchrotron periods to obtain the phase space plot shown in Fig. 64. The point was selected from the data 


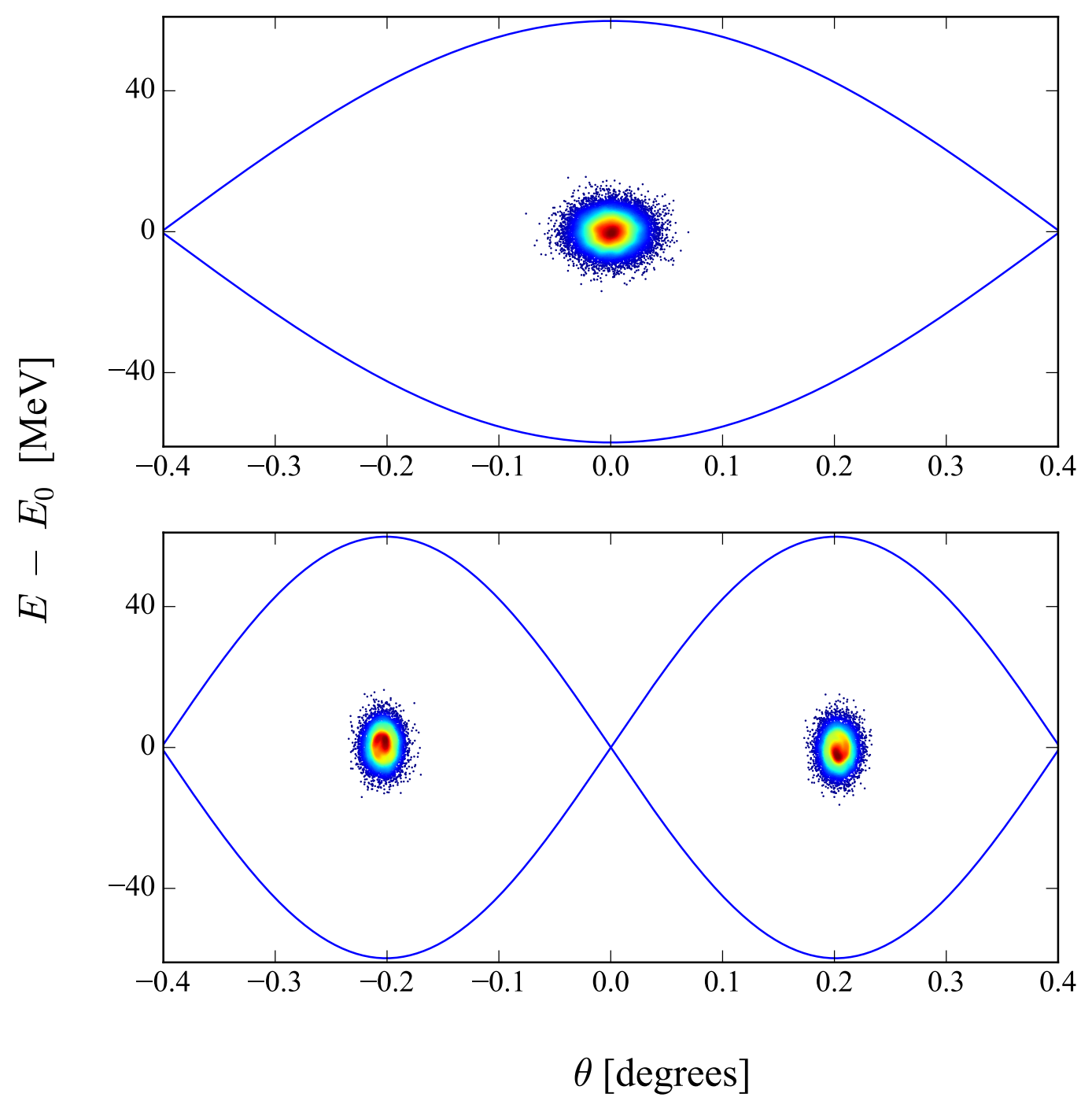

FIG. 64: Bunch splitting from the sample point from Fig. 63 .

A closer look at a single bunch after the split is shown in Fig. 65. The decoherence is larger compared to the bunch splitting results in Fig. 62, as indicated by the asymmetry in the spread of the particles, but this is expected for the low bunch to bucket ratios. 


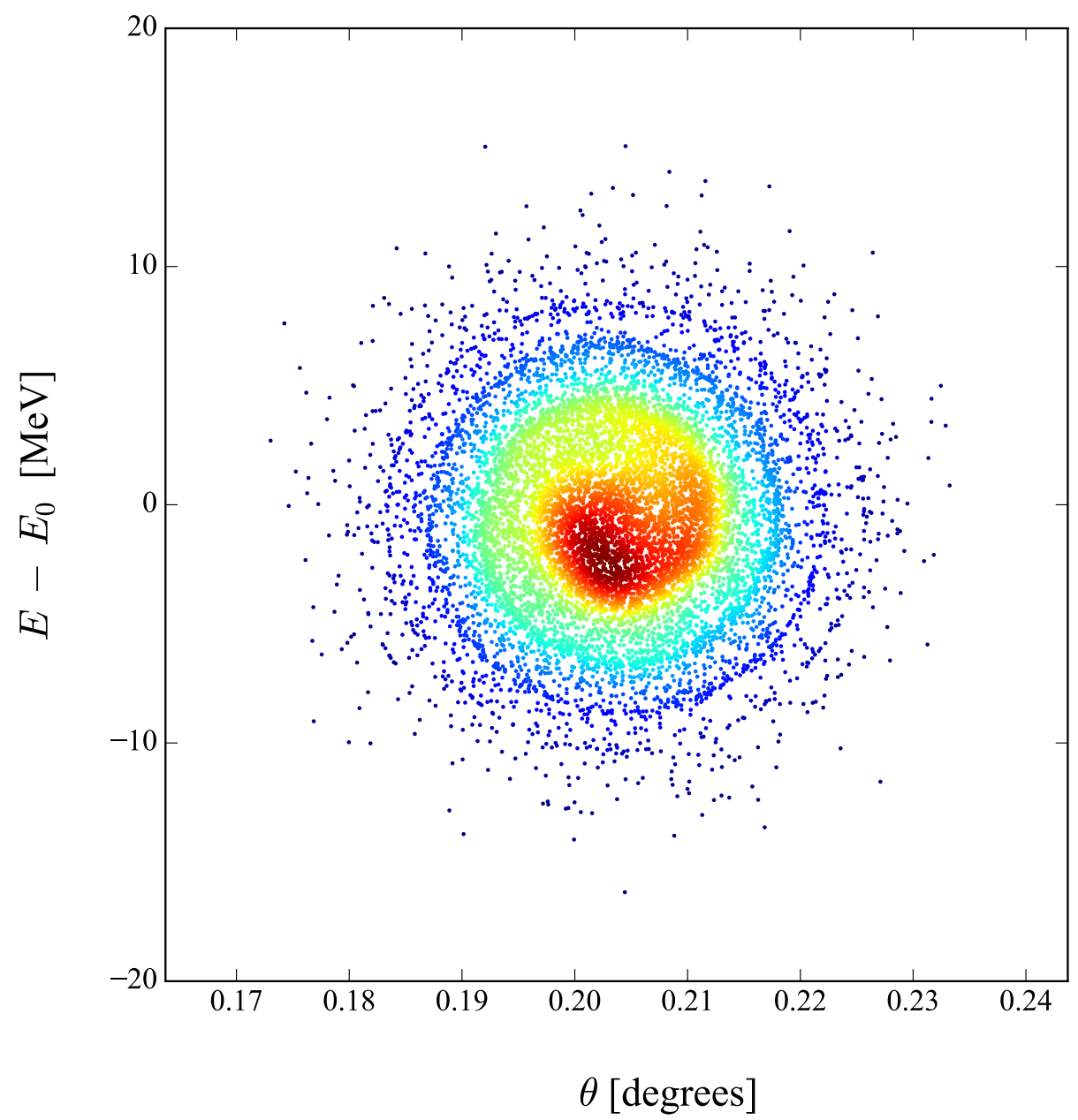

FIG. 65: A single bunch after the split with $T_{\text {split }}=85 T_{\mathrm{s}}$.

\subsubsection{LINEAR VOLTAGE CURVES WITH VARIABLE RAMPING TIMES AND VOLTAGE RATIO}

For the second group of simulations, the voltage ratio was added as a variable parameter in addition to the ones from the previous section. The voltage ratio was constrained to

$$
1.0 \leq \frac{V_{2}}{V_{1}} \leq 2.0 .
$$

Adding the voltage as a parameter makes the process non repeatable in successive bunch splits, but it is worth investigating for a system that may only require one split. Again, results from a simulation with a bunch to bucket ratio of 0.3 is presented first in Fig. 66 . 


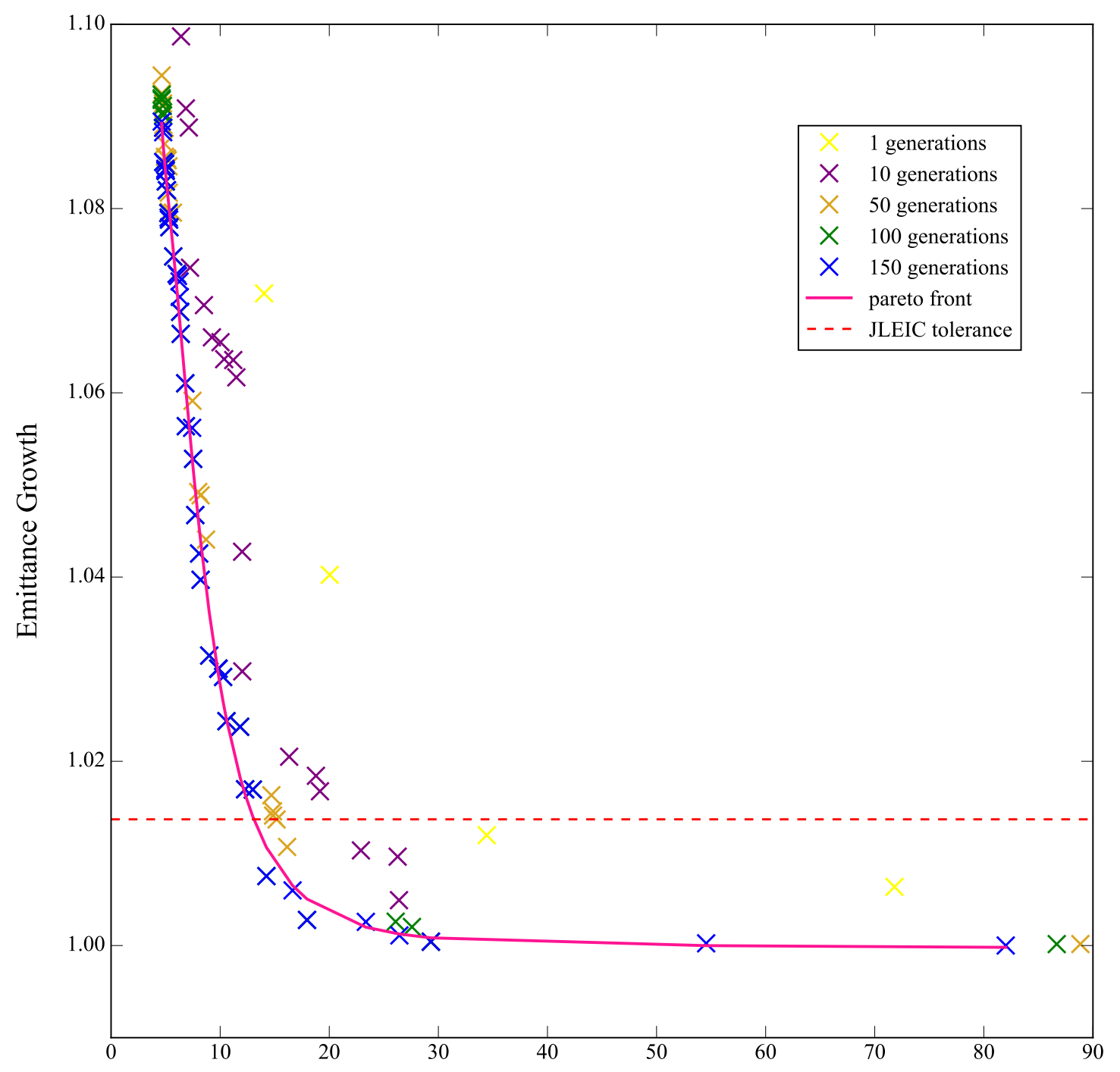

Split Time [Synchrotron periods]

FIG. 66: Pareto optimal curve for linear voltage variation with a bunch to bucket ratio 0.3.

The results for the 0.3 ratio show that the Pareto-optimal curve is almost identical to the case where the voltage ratio was kept constant, where an emittance growth of $1 \%$ is reached closer to 15 synchrotron periods.

Following these results, the simulation was repeated with a bunch to bucket ratio of 0.05 , and the Pareto-optimal curve is shown in Fig. 67. 


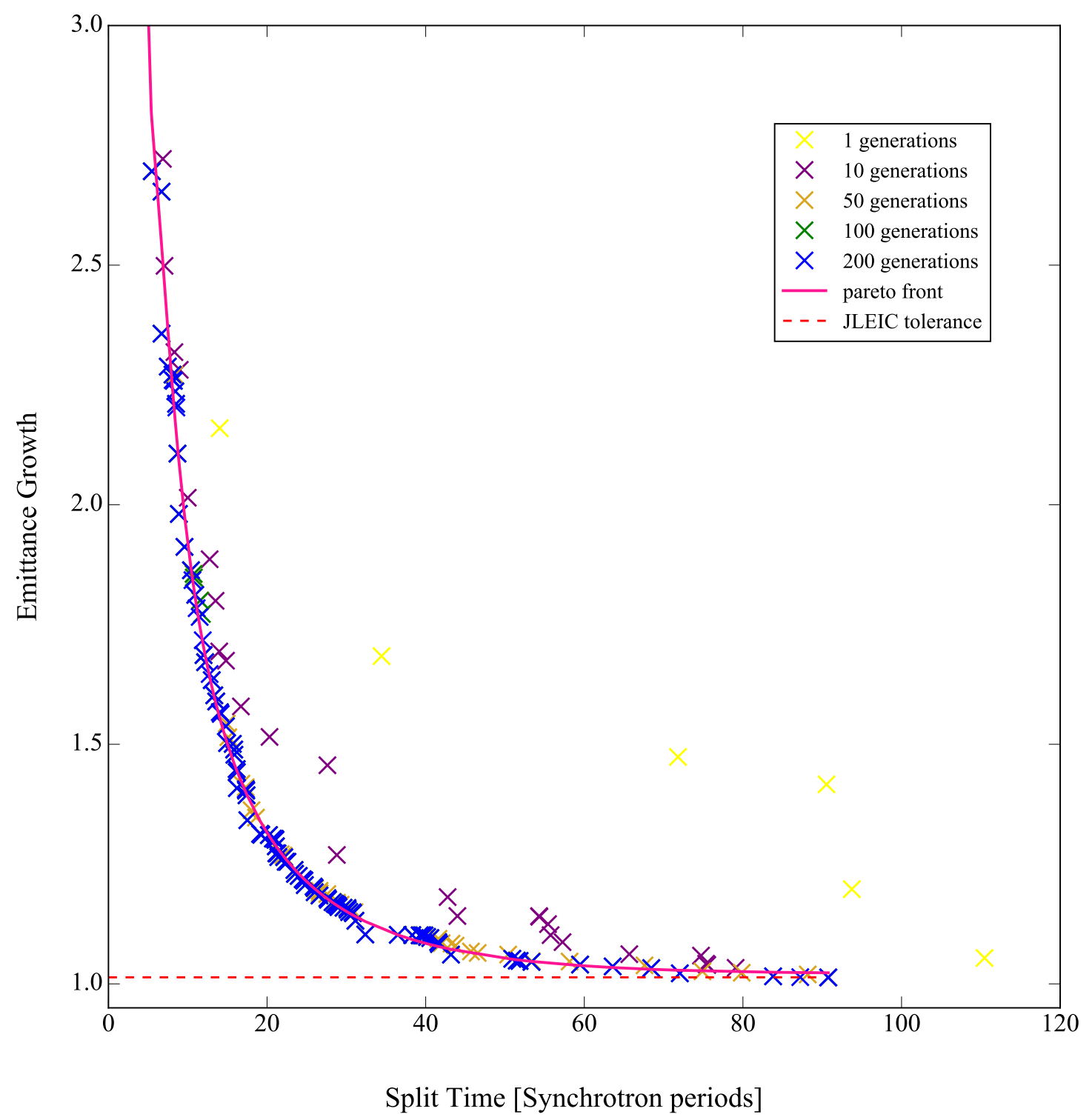

FIG. 67: Pareto optimal curve for linear RF voltage amplitude variation with a bunch to bucket ratio 0.05 .

A smaller bunch to bucket ratio with a variable emittance ratio did in fact showed a slight improvement over the fixed voltage case seen in Fig. 63. But it is still not significant enough to make the overall split time shorter.

\subsubsection{ISO-ADIABATIC VOLTAGE CURVES WITH VARIABLE RAMPING TIMES AND VOLTAGE RATIO}

Iso-adiabatic ramps are used for single harmonic systems because it was obtained through 
the adiabaticity parameter. However, bunch splitting is a process that has two active RF systems the entire time the tune changes during the split. The iso-adiabatic ramps considered here were calculated using the parameters of the RF source 1. An iso-adiabatic RF voltage amplitude ramp and the corresponding linear ramp for the same time period is shown in Fig. 19

The results of the GA simulation for a 0.3 bunch to bucket ratio are shown in Fig. 68

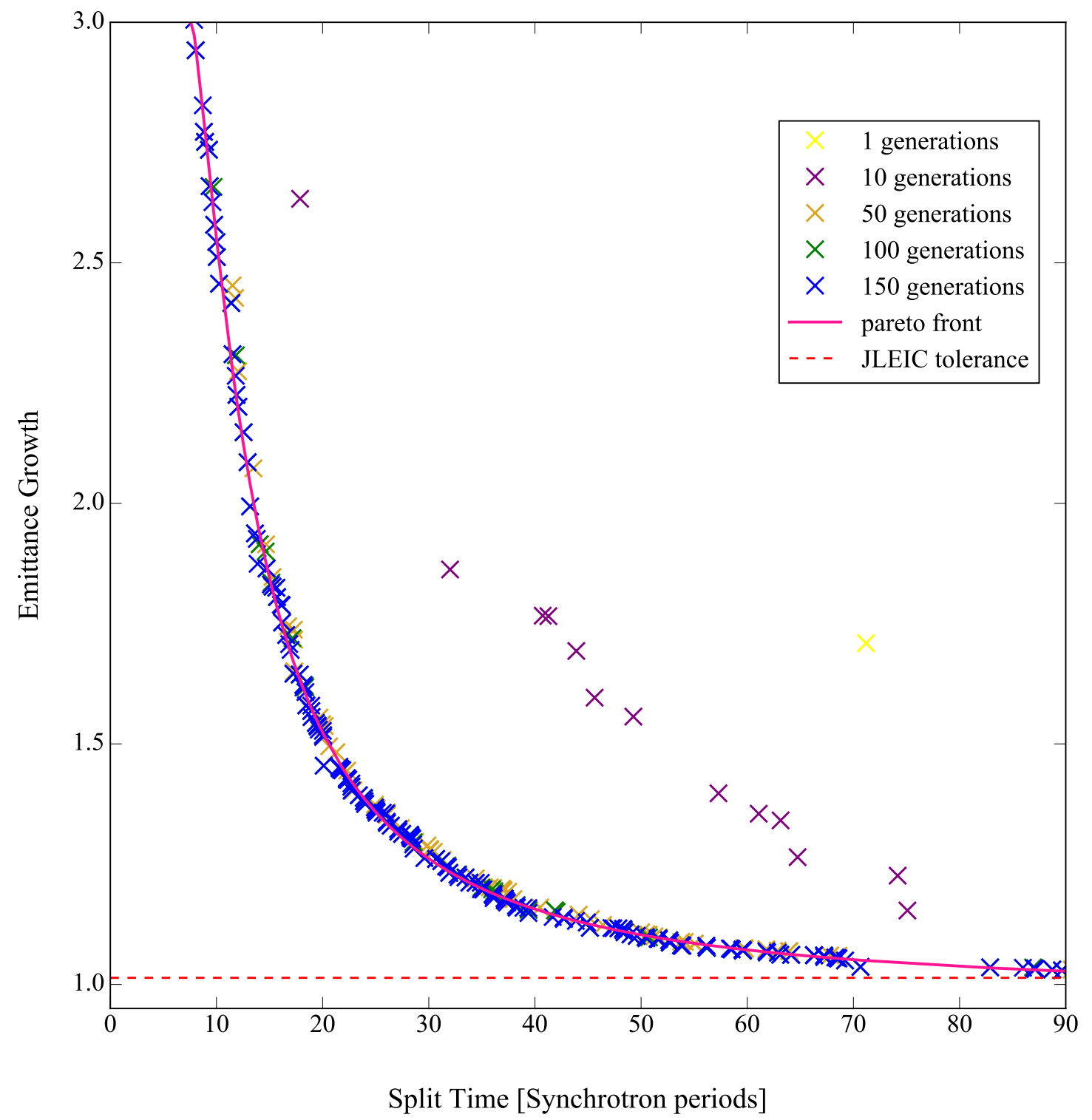

FIG. 68: Pareto optimal curve for iso-adiabatic voltage variation with a bunch to bucket ratio of 0.3 .

The results show that iso-adiabatic curves did not further improve the splitting time 
below the time achieved by the linear ramping curves from section subsection 7.3.1. This is due to having less RF control in between the two ramps and particles escaping from the bucket due to low momentum acceptance. This was repeated for the low-end bunch to bucket ratio of 0.05 , which similarly is not able to achieve the same reduced splitting times as the linear curves.

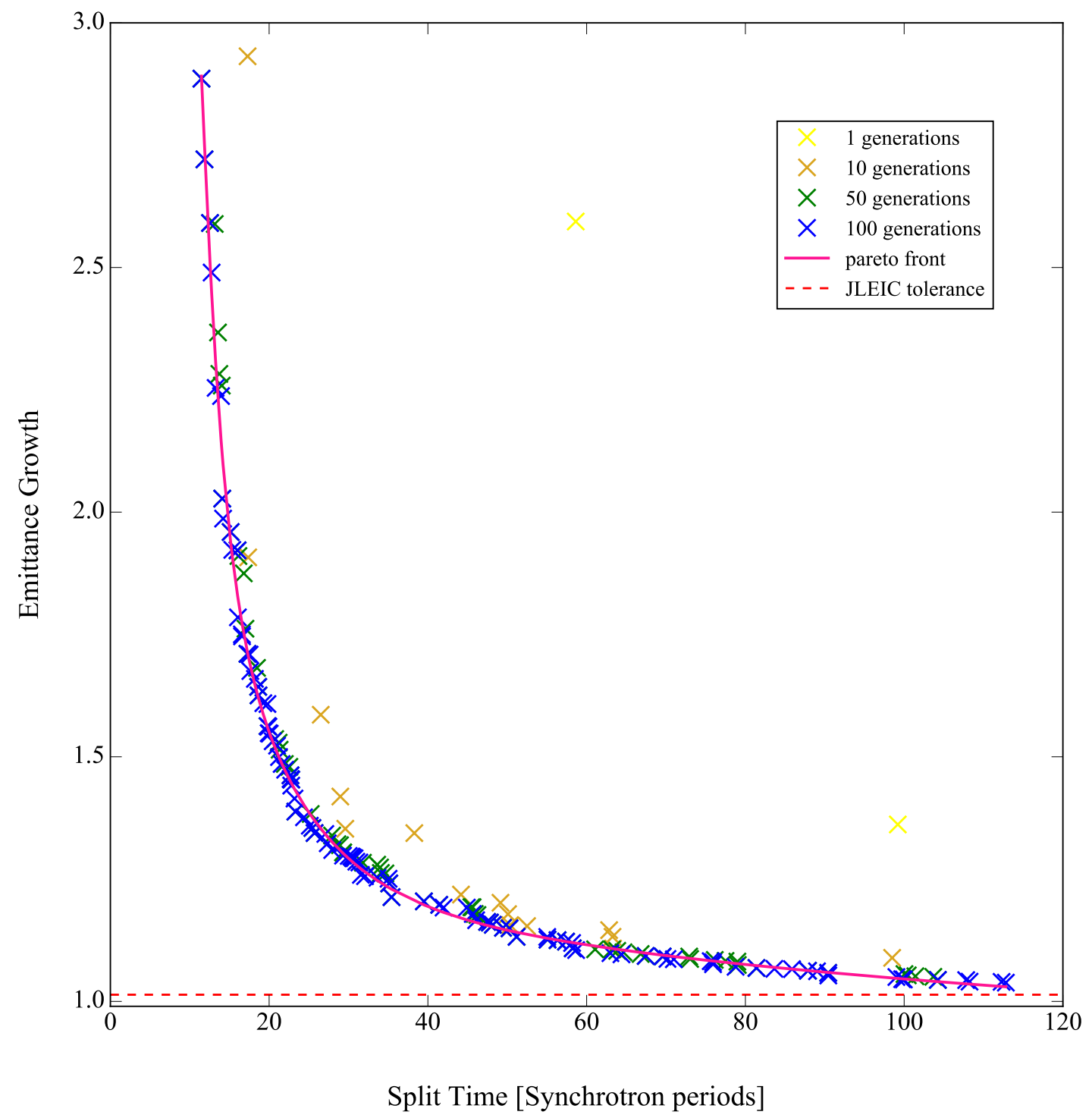

FIG. 69: Pareto optimal curve for iso-adiabatic voltage variation with a bunch to bucket ratio of 0.05 .

These results shows that using iso-adiabatic curves does not necessarily lower the emittance growth compared to the linear voltage curves, specially for higher bunch to bucket ratio. 


\section{CHAPTER 8}

\section{SUMMARY}

This dissertation addresses a fundamental challenge for JLEIC: how to form the short hadron bunches from long bunches accelerated through the ion complex. To achieve the required specifications for the high luminosity of JLEIC, a very high number of bunches (3328) is required before collision.

Due to the requirements of equal bunch emittances and intensities, two methods were considered: adiabatic debunch/rebunch and bunch splitting. Adiabatic debunch/rebunch requires fewer RF cavities compared to the bunch splitting, which requires a minimum of eight families. Adiabatic debunch/rebunch could also support required abort gaps through the use of barrier buckets; the parameters necessary to produce two barrier buckets in JLEIC were evaluated and found to be within existing technology. However, we found that adiabatic debunching intrinsically cannot provide a coasting beam with an emittance growth below the JLEIC tolerance.

Bunch splitting require $8 \mathrm{RF}$ systems for binary splitting to provide the desired number of bunches for JLEIC. Parameter scans enabled us to understand the time scales and the bunch to bucket ratios that are required to achieve the required small emittance growth. Optimizing further using a genetic algorithm provided results that reduced the split time by more than half and still kept the emittance dilution low. Iso-adiabatic curves that played a major role in adiabatic debunching (or rebunching) were not beneficial for binary RF splitting. This may be due to the fact that the iso-adiabatic curves have a high gradient at the beginning for debunching (or at the end for rebunching) which results in areas with either too low RF control of the system or both acting at maximum value. More investigation is required to verify these results. Linear voltage curves provided adequate results for the bunch splitting. Adding the RF voltage amplitude as a variable did help to reduce the overall split time but this results in the process being not completely repeatable. Future work can be done to investigate using smoother curves.

For the high number of bunches needed in JLEIC, the bunch splitting method seems to be the most viable and appropriate for conceptual design. The simulations in this dissertation demonstrate the feasibility of this approach for pre-conceptual design of JLEIC. 
This dissertation work also considered optimization of a tunable magnetic bunch compressor for an inverse Compton source designed at Old Dominion University. The tunable magnetic bunch compressor design involves two separate designs which both deliver a high $M_{56}$. Both designs are compact enough to fit in a small room. The two designs have different overall bending angles, which depending on the need can be fit in the corner or along the beam line. The quadrupoles in both designs incorporate the fringe field effects from a commercial quadrupole available from RadiaBeam Technologies. 


\section{BIBLIOGRAPHY}

[1] A. Aprahamian, R. Atcher, H. Caines, G. Cates, J. Cizewski, V. Cirigliano, D. Dean, A. Deshpande, R. Ent, F. Fahey, et al., INSPIRE-1398831.

[2] A. Accardi, A. Deshpande, Z. E. Meziani, J. W. Qiu, et al., Eur. Phys. J. A52, 268 (2016), arXiv:1212.1701.

[3] Jefferson Lab Electron Ion Collider, https://www.jlab.org/jleic.

[4] K. Deitrick, Ph.D. thesis, Old Dominion University (2017).

[5] Jefferson Lab, https://www.jlab.org/about-jefferson-lab.

[6] C. W. Leemann, D. R. Douglas, and G. A. Krafft, Annual Review of Nuclear and Particle Science 51, 413 (2001), https://doi.org/10.1146/annurev.nucl.51.101701.132327

[7] S. Abeyratne, D. Barber, A. Bogacz, P. Brindza, Y. Cai, A. Camsonne, A. Castilla, P. Chevtsov, E. Daly, Y. Derbenev, et al., (2015), arXiv:1504.07961.

[8] J. D. Jackson, Classical electrodynamics, 3rd ed. (Wiley, New York, NY, 1999).

[9] T. P. Wangler, RF linear accelerators (Wiley, 2008).

[10] M. Borland, elegant: A Flexible SDDS-Compliant Code for Accelerator Simulation, Advanced Photon Source LS-287, September 2000.

[11] M. Conte and W. W. MacKay, An introduction to the physics of particle accelerators; 2nd ed. (World Scientific, Singapore, 2008).

[12] G. W. Hill, Acta Math. 8, 1 (1886).

[13] A. W. Chao and M. Tigner, Handbook of Accelerator Physics and Engineering (World Scientific, Singapore, 1999).

[14] K. Steffen, High energy beam optics (Interscience Publishers, 1965).

[15] M. Syphers, http://home.fnal.gov/ syphers/Accelerators/tevPapers/LongEmitt.pdf.

[16] B. W. S. L. Montague, Single-particle dynamics-RF acceleration, (1977). 
[17] G. A. Krafft and G. Priebe, Reviews of Accelerator Science and Technology 03, 147 (2010), https://www.worldscientific.com/doi/pdf/10.1142/S1793626810000440.

[18] T. Satogata, K. Deitrick, J. Delayen, B. R. Gamage, K. Hernández-Chahín, C. Hopper, G. Krafft, and R. Olave, in Proceedings, 4th International Particle Accelerator Conference (IPAC 2013): Shanghai, China, May 12-17, 2013 (2013) p. WEPWA078.

[19] C. S. Hopper, K. E. Deitrick, and J. R. Delayen, in Proceedings, 2nd North American Particle Accelerator Conference (NA-PAC 2013): Pasadena, California, May 12-17, 2013 (2013) p. WEPAC42.

[20] P. Piot, Proceedings, LINAC 2004: ubeck, Germany, 2004, Conf. Proc. , 528 (2004).

[21] A. Loulergue and A. Mosnier, in Particle accelerator. Proceedings, 7th European Conference, EPAC 2000, Vienna, Austria, June 26-30, 2000. Vol. 1-3 (2001) pp. 752-754.

[22] M. Borland, "elegant: A flexible sdds-compliant code for accelerator simulation," (2000).

[23] D. Zhou, Y. Chen, J. Tang, and N. Wang, Proceedings, 1st International Particle Accelerator Conference (IPAC'10): Kyoto, Japan, May 23-28, 2010, Conf. Proc. C100523, THPD091 (2010).

[24] R. J. England, Ph.D. thesis, University of California, Los Angeles (2007).

[25] EMQD-01-155-245 specification sheet, http://www.radiabeam.com/upload/ catalog/pdf/136555330020130319_EMQD-01-155-245.pdf.

[26] J. Irwin and C.-X. Wang, in Proceedings Particle Accelerator Conference, Vol. 4 (1995) pp. 2376-2378 vol.4.

[27] R. Baartman and D. Kaltchev, in 2007 IEEE Particle Accelerator Conference (PAC) (2007) pp. 3229-3231.

[28] A. G. Ruggiero, Proceedings of the 1983 Particle Accelerator Conference (PAC 83): Accelerator Engineering and Technology, Santa Fe, New Mexico March 21-23, 1983, IEEE Trans. Nucl. Sci. 30, 2478 (1983).

[29] J. Griffin, J. MacLachlan, A. G. Ruggiero, and K. Takayama, IEEE Transactions on Nuclear Science 30, 2630 (1983). 
[30] J. Doskow, G. East, R. Ellis, Y. Jing, Y. Kim, S. Y Lee, T. Luo, P. Mcchesney, X. Pang, T. Rinckel, C. Romel, and P. Sokol, The ALPHA project at IU CEEM, (2010).

[31] Center for Exploration of Energy and Matter, https://ceem.indiana.edu/.

[32] T. Luo, Ph.D. thesis, Indiana University (2011).

[33] S. Y. Lee and K. Y. Ng, Phys. Rev. E55, 5992 (1997).

[34] C. M. Bhat, (2011), arXiv:1208.5784 [physics.acc-ph].

[35] J. Harzheim, D. Domont-Yankulova, M. Frey, K. Groß, and H. Klingbeil, in Proceedings, 8th International Particle Accelerator Conference (IPAC 2017): Copenhagen, Denmark, May 14-19, 2017 (2017) p. WEPVA047.

[36] M. Fujieda, Y. Iwashita, A. Noda, Y. Mori, C. Ohmori, Y. Sato, M. Yoshii, M. Blaskiewicz, J. M. Brennan, T. Roser, K. S. Smith, R. Spitz, and A. Zaltsmann, in Proceedings of the 1999 Particle Accelerator Conference (Cat. No.99CH36366), Vol. 2 (1999) pp. 857-859 vol.2.

[37] L. D. Landau, J. Phys.(USSR) 10, 25 (1946), [Zh. Eksp. Teor. Fiz.16,574(1946)].

[38] J. L. Laclare, in 2nd CERN Accelerator School: General Accelerator Physics Gif-surYvette, France, September 3-14, 1984 (1986) pp. 377-414.

[39] E. Keil and W. Schnell, Concerning longitudinal stability in the ISR, Tech. Rep. CERNISR-TH-RF-69-48. ISR-TH-RF-69-48 (CERN, Geneva, 1969).

[40] D. Boussard, Observation of microwave longitudinal instabilities in the CPS, Tech. Rep. CERN-LabII-RF-INT-75-2. LabII-RF-INT-75-2 (CERN, Geneva, 1975).

[41] R. Garoby, in Proceedings, CAS - CERN Accelerator School: RF for Accelerators, Ebeltoft, Denmark, 8 - 17 Jun 2010 (2011) arXiv:1112.3232 [physics.acc-ph].

[42] R. Li, "Instabilities and collective effects," (2018), jLEIC Collaboration Meeting, Spring.

[43] F. Marhauser, (private communication) (2016).

[44] R. Garoby, in Proceedings, 17th International Conference on High-Energy Accelerators, HEACC 1998: Dubna, Russian Federation, September 07-12, 1998 (1998) pp. 172-174. 
[45] J. Guo, "Jleic ion and electron beam formation: Schemes and parameters," (2016), fall 2016 JLEIC Collaboration Meeting, Jefferson Lab.

[46] I. Bozsik, I. Hofmann, A. Jahnke, and R. W. Müller, in Computing in Accelerator Design and Operation, edited by W. Busse and R. Zelazny (Springer Berlin Heidelberg, Berlin, Heidelberg, 1984) pp. 128-133.

[47] R. Garoby, IEEE Transactions on Nuclear Science 32, 2332 (1985).

[48] J. Brennan, in Proc. of the Third ICFA Mini-Workshop on High Intensity, High Brightness Hadron Accelerators, May 1997 (1997) pp. 3-23.

[49] PISA, https://sop.tik.ee.ethz.ch/pisa/?page=beginners.php.

[50] B. Terzić, A. S. Hofler, C. J. Reeves, S. A. Khan, G. A. Krafft, J. Benesch, A. Freyberger, and D. Ranjan, Phys. Rev. ST Accel. Beams 17, 101003 (2014).

[51] A. Hofler, B. Terzic, M. Kramer, A. Zvezdin, V. Morozov, Y. Roblin, F. Lin, and C. Jarvis, Phys. Rev. ST Accel. Beams 16, 010101 (2013). 


\title{
VITA
}

\author{
Bamunuvita Randika Prasad Gamage \\ Department of Physics \\ Old Dominion University \\ Norfolk, VA 23529
}

Randika Gamage was born on July 15 in Colombo, Sri Lanka. He was awarded a Bachelor of Science degree in Computational Physics from University of Colombo, Sri Lanka in 2010. Randika joined the Department of Physics at Old Dominion University in Norfolk, Virginia, USA in August 2011 and completed his Masters of Science degree in Physics in August of 2013.

Typeset using IATEX. 